\title{
INTERACTION DE DEUX CHOCS POUR UN SYSTEME DE DEUX LOIS DE CONSERVATION, EN DIMENSION DEUX D'ESPACE
}

\author{
GUY METIVIER
}

\begin{abstract}
The existence of shock front solutions to a system of conservation laws in several space variables has been proved by A. Majda, solving a Cauchy problem, with a suitable discontinuous Cauchy data. But, in general, the solution to such a Cauchy problem will present $N$ singularities, $N$ being the number of laws. In this paper we solve (locally) this Cauchy problem, with a Cauchy data which is piecewise smooth, in the case where all the singularities are expected to be shock waves. Actually the construction is written for a system of two laws, with two space variables and similarly, for such a system, the same method enables us to study the interaction of two shock waves. The key point, in the construction below, is the study of a nonlinear, free boundary Goursat problem.
\end{abstract}

I. Introduction. Dans [8] A. Majda a construit des chocs pour un système de lois de conservation:

$$
\frac{\partial u}{\partial t}+\sum_{j=1}^{n} \frac{\partial}{\partial x_{j}} f_{j}(u)=0
$$

en résolvant le problème de Cauchy pour une donnée initiale discontinue à travers une hypersurface, convenablement choisie. Mais, en général, la solution de ce problème de Cauchy va présenter $N$ singularités, ondes de choc ou de raréfaction, issues de la discontinuité initiale ( $N$ étant la dimension du système). Dans ce travail, on a d'abord voulu mener au bout la résolution de ce problème de Cauchy, dans le cas où l'on s'attend á ce que la solution ne présente que des chocs, et dans le cas le plus simple d'un système $2 \times 2$ en dimension d'espace $n=2$ On obtiendrait un résultat analogue pour des dimensions supérieures, en remarquant toutefois que les hypothèses de stabilité imposées, requièrent que $n \leqslant N$. Par ailleurs, la présence d'ondes de raréfaction sera étudiée ultérieurement.

De même que $\mathrm{A}$. Majda ramène la construction d'un choc, à un problème mixte hyperbolique à frontière libre, nous ramènerons la construction de ces deux chocs issus d'une même surface initiale, à la résolution d'un problème de Goursat non linéaire, à frontière libre.

Le deuxième but de ce travail est d'étudier, dans mêmes conditions $N=n=2$, l'interaction ou le croisement de deux chocs. Ce problème relève des mêmes techniques que le précédent, et on le ramènera lui aussi, au même type de problème de Goursat.

Received by the editors January 25, 1985 .

1980 Mathematics Subject Classification. Primary 35L65, 35L50; Secondary 76L05.

(C1986 American Mathematical Society $0002-9947 / 86 \$ 1.00+\$ .25$ per page 
La démarche adoptée est la suivante: on part d'une situation modèle, concernant des chocs plans, puis on perturbe cette situation, grâce à des hypothèses de stabilité. En particulier, la notion de stabilité uniforme des chocs, introduite par A. Majda [7], est primordiale pour ce travail. Cette démarche est particulièrement justifiée dans l'étude des chocs faibles pour un système strictement hyperbolique, vraiment non linéaire; en effet, dans ce cas, l'étude des chocs plans est entièrement faite par P. D. Lax [5], alors que les hypothèses de stabilité sont automatiquement satisfaites (cf. Appendice A).

Le plan de ce travail est le suivant: au paragraphe 2 on énonce les résultats concernant le problème de Cauchy et le problème d'interaction. Au paragraphe 3, la démonstration de ces résultats est ramenée à celle d'un théorème d'existence pour un problème de Goursat dans un dièdre; ce théorème est énoncé au paragraphe 3.3. La construction de la solution se fait selon le schéma suivant: par développement de Taylor le long de l'arête du dièdre, on construit d'abord une "solution approchée". Le paragraphe 4 prépare à cette construction. On est alors ramené à résoudre le problème de Goursat dans des espaces de fonctions plates sur l'arête. Les résultats techniques concernant ces espaces sont regroupés au paragraphe 5 . On étudie alors un problème linéarisé (paragraphe 6), et on conclut par un schéma itératif (paragraphe 7), semblable à celui utilisé par A. Majda [8].

\section{2. Ènonce des résultats.}

2.1 Problème de Cauchy. Considérons un système de la forme:

$$
\frac{\partial u}{\partial t}+\frac{\partial}{\partial x} f(u)+\frac{\partial}{\partial y} g(u)=0
$$

où l'inconnue $u=\left(u_{1}, u_{2}\right)$ est une fonction des variables $(t, x, y) \in \mathbf{R}^{3}$, à valeurs dans $\mathbf{R}^{2}$, et où $f$ et $g$ sont des fonctions $C^{\infty}$ de $\mathbf{R}^{2}$ dans lui-même. Nous noterons $A(u)$ et $B(u)$ les matrices $f^{\prime}(u)$ et $g^{\prime}(u)$, et dans toute la suite nous ferons l'hypothèse de stricte hyperbolicité suivante:

$\left(\mathrm{H}_{0}\right) \quad$ pour tout $u \in \mathbf{R}^{2}$, tout $(\xi, \eta) \in \mathbf{R}^{2} \backslash 0$ la matrice $\xi A(u)$ $+\eta B(u)$, a deux valeurs propres réelles distinctes.

Nous posons d'abord le problème de Cauchy pour (2.1.1) avec une donnée initiale:

$$
\left.u\right|_{t=0}=u_{0}
$$

discontinue à travers une courbe $S_{0}$. Nous nous plaçons sur un voisinage $\omega \subset \mathbf{R}^{2}$ d'un point $\left(x_{0}, y_{0}\right) \in S_{0}$, partagé en deux composantes $\omega_{+}$et $\omega_{-}$et nous notons $u_{0}^{+}$ les restrictions de $u_{0}$ à $\omega_{ \pm}$. Nous supposons que $S_{0}$ est $C^{\infty}$ et que les fonctions $u_{0}^{+}$ sont $C^{\infty}$ sur $\bar{\omega}_{ \pm}$. Pour fixer les idées on peut choisir les coordonnées de sorte que $\left(x_{0}, y_{0}\right)=0, . S_{0}$ soit d'équation $x=\varphi_{0}(y)$ avec $\varphi_{0}(0)=\varphi_{0}^{\prime}(0)=0$, et que $\omega_{ \pm}=$ $\left\{(x, y) \in \omega \mid \pm\left(x-\varphi_{0}(y)\right)>0\right\}$.

Notre but est de donner des conditions raisonnables qui permettent de construire une solution de (2.1.1), (2.1.2) sous forme de deux ondes de choc issues de la cuirbe $S_{0}$. De façon plus précise, nous cherchons, au voisinage de l'origine, des fonctions 
$\varphi(t, y)$ et $\Psi(t, y)$ telles que:

$$
\left\{\begin{array}{l}
\varphi(t, y)<\Psi(t, y) \quad \text { pour } t>0 \\
\varphi(0, y)=\Psi(0, y)=\varphi_{0}(y)
\end{array}\right.
$$

et des fonctions régulières $u^{+}, u^{-}$et $u^{\#}$, telles que la fonction $u$ suivante, soit solution faible de (2.1.1), (2.1.2):

$$
u(t, x, y)= \begin{cases}u^{-}(t, x, y) & \text { pour } x<\varphi(t, y), \\ u^{\#}(t, x, y) & \text { pour } \varphi(t, y)<x<\Psi(t, y), \\ u^{+}(t, x, y) & \text { pour } \Psi(t, y)<x\end{cases}
$$

En clair, cela veut dire, d'une part, que $u^{+}, u^{-}, u^{\#}$ sont solutions classiques de (2.1.1) dans les ouverts respectifs définis en (2.1.4), avec $\left.u^{ \pm}\right|_{t=0}=u_{0}^{ \pm}$; d'autre part, que sont satisfaites les conditions de saut de Rankine-Hugoniot:

$$
\begin{cases}\left(u^{\#}-u^{-}\right) \frac{\partial \varphi}{\partial t}+\left[g\left(u^{\#}\right)-g\left(u^{-}\right)\right] \frac{\partial \varphi}{\partial y}=f\left(u^{\#}\right)-f\left(u^{-}\right) & \text {pour } x=\varphi(t, y), \\ \left(u^{+}-u^{\#}\right) \frac{\partial \Psi}{\partial t}+\left[g\left(u^{+}\right)-g\left(u^{\#}\right)\right] \frac{\partial \Psi}{\partial y}=f\left(u^{+}\right)-f\left(u^{\#}\right) & \text { pour } x=\Psi(t, y) .\end{cases}
$$

Enfin, on veut que $u$ satisfasse certaines conditions d'entropie, et on exige, au moins, que soient satisfaites les conditions de choc de $\operatorname{Lax}[5,6]$.

Posant

$$
\begin{aligned}
& \underline{u}^{ \pm}=u_{0}^{+}(0,0), \quad \underline{u}^{\#}=u^{\#}(0,0,0), \\
& \rho=\frac{\partial \varphi}{\partial t}(0,0) \quad \text { et } \quad \sigma=\frac{\partial \Psi}{\partial t}(0,0),
\end{aligned}
$$

(2.1.5) implique que

(2.1.6) $\rho\left(\underline{u}^{\#}-\underline{u}^{-}\right)=f\left(\underline{u}^{\#}\right)-f\left(\underline{u}^{-}\right), \quad \sigma\left(\underline{u}^{+}-\underline{u}^{\#}\right)=f\left(\underline{u}^{+}\right)-f\left(\underline{u}^{\#}\right)$.

En fait, si l'on pose

$$
\begin{gathered}
\underline{u}_{0}(x, y)= \begin{cases}\underline{u}^{-} & \text {pour } x<0, \\
\underline{u}^{+} & \text {pour } x>0,\end{cases} \\
\underline{u}(t, x, y)= \begin{cases}\underline{u}^{-} & \text {pour } x<\rho t, \\
\underline{u}^{\#} & \text { pour } \rho t<x<\sigma t, \\
\underline{u}^{+} & \text {pour } \sigma t<x,\end{cases}
\end{gathered}
$$

alors (2.1.6) signifie que $\underline{u}$ est solution faible du problème de Cauchy modèle, obtenu en remplaçant la donnée de Cauchy $u_{0}$ par $\underline{u}_{0}$.

Notant $\lambda_{i}(u)$ et $r_{i}(u)$, pour $i=1,2$, les valeurs propres et vecteurs propres correspondants de la matrice $A(u)\left(\lambda_{1}<\lambda_{2}\right)$, les conditions de chocs de Lax [5] pour $\underline{u}$ s'écrivent:

$$
\begin{cases}\lambda_{1}\left(\underline{u}^{\#}\right)<\rho<\lambda_{1}\left(\underline{u}^{-}\right), & \rho<\lambda_{2}\left(\underline{u}^{\#}\right) \\ \lambda_{2}\left(\underline{u}^{+}\right)<\sigma<\lambda_{2}\left(\underline{u}^{\#}\right), & \sigma>\lambda_{1}\left(\underline{u}^{\#}\right)\end{cases}
$$


Pour résoudre le problème (2.1.1), (2.1.2) nous sommes donc amenés à formuler l'hypothèse suivante:

$$
\begin{aligned}
& \text { posant } \underline{u}^{+}=u_{0}^{+}(0) \text {, il existe } \underline{u}^{\#} \in \mathbf{R}^{2}, \rho<\sigma \text { tels que l'on ait } \\
& \text { (2.1.6) (2.1.9). }
\end{aligned}
$$

Remarque 2.1.1. Cette hypothèse et le fait même, que l'on cherche u sous la forme (2.1.4), sont motivés par le travail de P. D. Lax [5] qui a montré que si $\underline{u}^{+}$et $\underline{u}^{-}$ restent dans un compact, pour $\left|\underline{u}^{+}-\underline{u}^{-}\right|$assez petits on peut trouver $\rho, \sigma$ et $\underline{u}^{\#}$ vérifiant (2.1.6); en outre, si $A(u)$ est vraiment non linéaire (c'est à dire si $r_{i} \operatorname{grad}_{u} \lambda_{i} \neq 0$ pour $\left.i=1,2\right),(2.1 .9)$ est vérifié poứ, grossièrement parlant, un quart des $\left(\underline{u}^{+}, \underline{u}^{-}\right)$(dans les autres cas la solution d'entropie de (2.1.1) avec donnée initiale $\underline{u}_{0}$, ne présente qu'un seul choc, ou alors au moins une onde de raréfaction).

L'idée, maintenant, est de construire $u$ comme une "perturbation" de $\underline{u}$; cela conduit à faire deux hypothèses de stabilitè; la première est la suivante:

$\left(\mathrm{H}_{2}\right) \quad$ Les chocs plans de la fonction $\underline{u}$ (2.1.8), sont uniformément, stables au sens de A. Majda [7].

Cela veut dire (cf. [7]), que pour les problèmes mixtes suivants:

$$
\begin{aligned}
& \left\{\begin{array}{l}
\frac{\partial v}{\partial t}+\left(A\left(\underline{u}^{\#}\right)-\rho\right) \frac{\partial v}{\partial x}+B\left(\underline{u}^{\#}\right) \frac{\partial v}{\partial y}=f \quad \text { pour } x>0, \\
\left(\underline{u}^{\#}-\underline{u}^{-}\right) \frac{\partial \Phi}{\partial t}+\left[g\left(\underline{u}^{\#}\right)-g\left(\underline{u}^{-}\right)\right] \frac{\partial \phi}{\partial y}-\left(A\left(\underline{u}^{\#}\right)-\rho\right) v_{\mid x=0}=g,
\end{array}\right. \\
& \left\{\begin{array}{l}
\frac{\partial v}{\partial t}+\left(A\left(\underline{u}^{\#}\right)-\sigma\right) \frac{\partial v}{\partial x}+B\left(\underline{u}^{\#}\right) \frac{\partial v}{\partial y}=f \quad \text { pour } x<0, \\
\left(\underline{u}^{+}-\underline{u}^{\#}\right) \frac{\partial \Phi}{\partial t}+\left[g\left(\underline{u}^{+}\right)-g\left(\underline{u}^{\#}\right)\right] \frac{\partial \phi}{\partial y}-\left(A\left(\underline{u}^{\#}\right)-\sigma\right) v_{\mid x=0}=g,
\end{array}\right.
\end{aligned}
$$

la condition de Lopatinski uniforme est satisfaite; on renvoie à [7] où à l'appendice A pour une explicitation de cette condition. La seconde hypothèse de stabilité se formule de la façon suivante:

Si l'on note $\left(\begin{array}{c}\alpha \\ \beta\end{array}\right)$ et $\left(\begin{array}{l}\gamma \\ \delta\end{array}\right)$ les composantes de $\left(A\left(\underline{u}^{\#}\right)-\rho\right)^{-1}\left(\underline{u}^{\#}-\underline{u}^{-}\right)$,

$\left(\mathrm{H}_{3}\right) \quad$ et $\left(A\left(\underline{u}^{\#}\right)-\sigma\right)^{-1}\left(\underline{u}^{\#}-\underline{u}^{+}\right)$dans la base $r_{1}\left(\underline{u}^{\#}\right), r_{2}\left(\underline{u}^{\#}\right)$, alors $|\beta \gamma|<|\alpha \delta|$.

Remarque 2.1.2. Si $A(u)$ est vraiment non linéaire, dans le cas de chocs faibles, les hypothèses $\left(\mathrm{H}_{2}\right)$ et $\left(\mathrm{H}_{3}\right)$ sont toujours satisfaites (cf. Appendice $\left.\mathrm{A}\right)$.

ThEORÈm 2.1.3. Sous les hypothèses $\left(\mathrm{H}_{0}\right)$ à $\left(\mathrm{H}_{3}\right)$, il existe des fonctions $\varphi, \Psi$, $u^{-}, u^{\#}$ et $u^{+}$, définies et de classe $C^{1}$ au voisinage de l'origine, vérifiant (2.1.3), et telle que la fonction $u$ définie pour $t>0$ par (2.1.4) soit solution faible du problème de Cauchy (2.1.1) (2.1.2).

2.2. Interaction de deux chocs. Etudions d'abord le cas de deux chocs plans, qui, au temps $t=0$, se coupent suivant la droite $x=0$ : on se donne dans le passé $t<0$, 
une solution faible de (2.1.1) sous la forme

$$
u(t, x, y)= \begin{cases}\underline{u}^{-} & \text {pour } x<\sigma_{1} t, \\ \underline{u}^{b} & \text { pour } \sigma_{1} t<x<\rho_{1} t, \\ \underline{u}^{+} & \text {pour } \rho_{1} t<x,\end{cases}
$$

où $\underline{u}^{-}, \underline{u}^{b}, \underline{u}^{+}$sont des vecteurs de $\mathbf{R}^{2}$, deux à deux différents, et où $\rho_{1}<\sigma_{1}$. On suppose que sont satisfaites les conditions de choc de Lax.

Prolonger $\underline{u}$ pour $t>0$ revient exactement à résoudre le problème de Cauchy avec la donnée initiale (2.1.7). Le lemme suivant résulte immédiatement de [5]

LEMME 2.2.1. Supposons $A(u)$ vraiment non linéaire. Etant donné un compact $K$ de $\mathbf{R}^{2}$ et un réel $C$, il existe $\varepsilon>0$ tel que si les chocs de $(2.2 .1)$ vérifient $\left(\underline{u}^{-}, \underline{u}^{b}, \underline{u}^{+}\right) \in K^{3}$ et

$$
\frac{1}{C}\left|\underline{u}^{+}-\underline{u}^{b}\right| \leqslant\left|\underline{u}^{b}-\underline{u}^{-}\right| \leqslant C\left|\underline{u}^{+}-\underline{u}^{b}\right|<\varepsilon
$$

alors il existe $\underline{u}^{\#}, \rho<\sigma$ satisfaisant à (2.1.6) (2.1.9).

Cela signifie que si les chocs incidents sont d'intensité comparable et suffisemment faibles, alors, dans l'avenir $(t>0)$, on a deux autres chocs; on peut représenter la fonction $\underline{u}$ par la figure suivante:

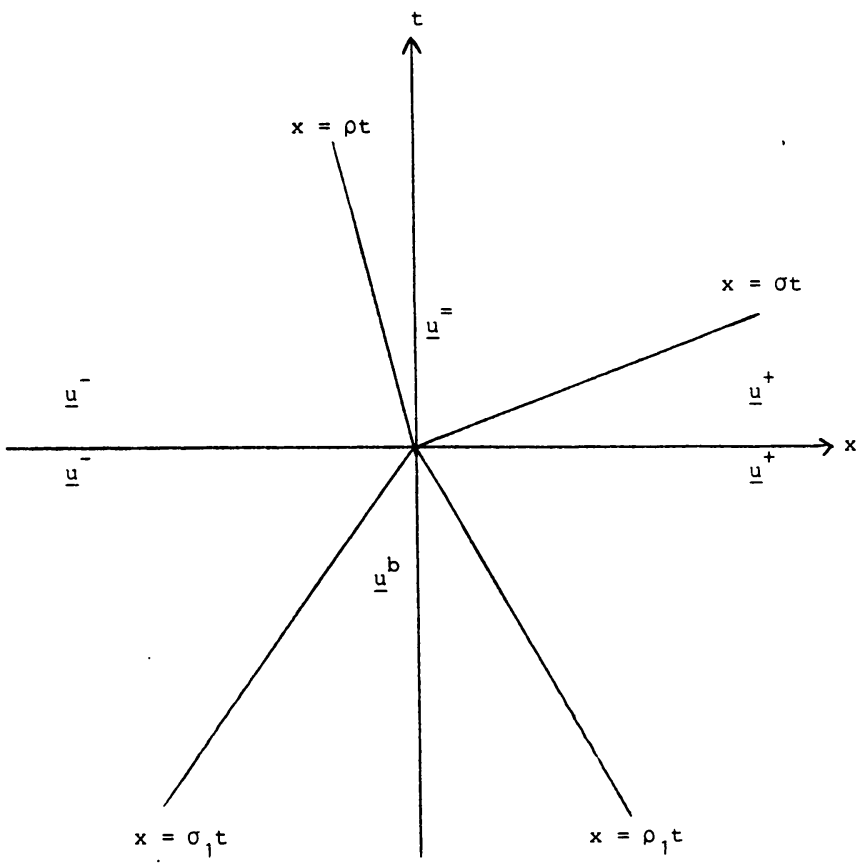


Nous perturbons maintenant ce schéma, en nous donnant dans le passé $t<0$ une solution faible $u$, de (2.1.1), présentant deux chocs $R_{1}$ et $S_{1}$, d'équations $x=\varphi_{1}(t, y)$ et $x=\Psi_{1}(t, y)$ avec $\psi_{1}<\varphi_{1}$, de la forme suivante:

$$
u(t, x, y)= \begin{cases}u^{-}(t, x, y) & \text { pour } x<\Psi_{1}(t, y), \\ u^{b}(t, x, y) & \text { pour } \Psi_{1}(t, y)<x<\varphi_{1}(t, y), \\ u^{+}(t, x, y) & \text { pour } \varphi_{1}(t, y)<x\end{cases}
$$

On suppose que les fonctions sont régulières jusqu'en $t=0$ (au voisinage de l'origine) et que les surfaces $R_{1}$ et $S_{1}$ se coupent transversalement à l'origine; on peut supposer que $\Psi_{1}=\varphi_{1}=\partial \Psi_{1}^{1} / \partial y=\partial \varphi_{1} / \partial y=0$ à l'origine; posant $\rho_{1}=$ $\left(\partial \varphi_{1} / \partial t\right)(0,0), \sigma_{1}=\left(\partial \Psi_{1} / \partial t\right)(0,0)$, on a alors $\rho_{1}<\sigma_{1}$. Par ailleurs, notons $\underline{u}^{+}, \underline{u}^{b}$ et $\underline{u}^{-}$les valeurs à l'origine des fonctions correspondantes; alors la fonction $\underline{u}$ (2.2.1) est elle aussi solution faible de (2.2.1). Guidés par le Lemme 2.2.1, nous sommes amenés à faire l'hypothèse suivante:

(i) Les chocs de (2.2.1) sont uniformément stables au sens

$$
\text { de A. Majda [7]. }
$$

(ii) Il existe $\underline{u}^{\#}, \rho<\sigma$, tels que l'on ait (2.1.6), (2.1.9).

Par ailleurs nous supposerons aussi que les hypothèses $\left(\mathrm{H}_{2}\right)$ et $\left(\mathrm{H}_{3}\right)$ sont satisfaites.

On veut alors prolonger la solution $u$ pour $t>0$, de la manière suivante: on prolonge d'abord les fonctions $u^{-}, u^{b}, u^{+}, \varphi_{1}$ et $\Psi_{1}$; les surfaces $R_{1}$ et $S_{1}$ ainsi prolongées vont se couper suivant une courbe $S_{0}$ que l'on peut paramétrer de la manière suivante:

$$
t=\tau(y) ; \quad x=\varphi_{1}(t, y)=\Psi_{1}(t, y)
$$

On aura donc

$$
\begin{cases}\tau(y) \geqslant 0, & \\ \varphi_{1}(t, y)>\Psi_{1}(t, y) & \text { pour } t<\tau(y), \\ \varphi_{1}(t, y)=\Psi_{1}(t, y) & \text { pour } t=\tau(y)\end{cases}
$$

Pour $t>\tau(y)$, on cherche la solution $u$ sous la forme:

$$
u(t, x, y)= \begin{cases}u^{-}(t, x, y) & \text { pour } x<\varphi(t, y), \\ u^{\#}(t, x, y) & \text { pour } \varphi(t, y)<x<\Psi(t, y), \\ u^{+}(t, x, y) & \text { pour } \Psi(t, y)<x,\end{cases}
$$

les fonctions $\varphi$ et $\Psi$ vérifiant:

$$
\left\{\begin{array}{l}
\varphi(t, y)<\Psi(t, y) \quad \text { pour } t>\tau(y) \\
\varphi(t, y)=\Psi(t, y)=\varphi_{1}(t, y)=\Psi_{1}(t, y) \quad \text { pour } t=\tau(y)
\end{array}\right.
$$

THEORĖME 2.2.2. Sous les hypothèses $\left(\mathrm{H}_{0}\right),\left(\mathrm{H}_{1}^{\prime}\right),\left(\mathrm{H}_{2}\right)$ et $\left(\mathrm{H}_{3}\right)$, il existe, au voisinage de l'origine, des prolongements réguliers de $u^{-}, u^{b}, u^{+}, \varphi_{1}$ et $\Psi_{1}$, des fonctions $u^{\#}, \tau, \varphi$ et $\Psi$ de classe $C^{1}$, vérifiant (2.2.4) et (2.2.6), de sorte que la fonction u définie par (2.2.2) pour $t<\tau(y)$ et par (2.2.5) pour $t>\tau(y)$, soit solution faible de (2.1.1). 
REMARQue 2.2.3. Si le système (2.2.1) est strictement hyperbolique (vérifie $\left(\mathrm{H}_{0}\right)$ ) et si $A(u)$ est vraiment non linéaire, il résulte du Lemme 2.2.1 et de l'appendice $\mathrm{A}$, que les hypothèses $\left(\mathrm{H}_{1}^{\prime}\right),\left(\mathrm{H}_{2}\right)$ et $\left(\mathrm{H}_{3}\right)$ sont satisfaites pour des chocs incidents d'intensité comparable, suffisemment faibles.

Terminons ce paragraphe en donnant une représentation de la solution (2.2.2), (2.2.5) à l'aide du film suivant:
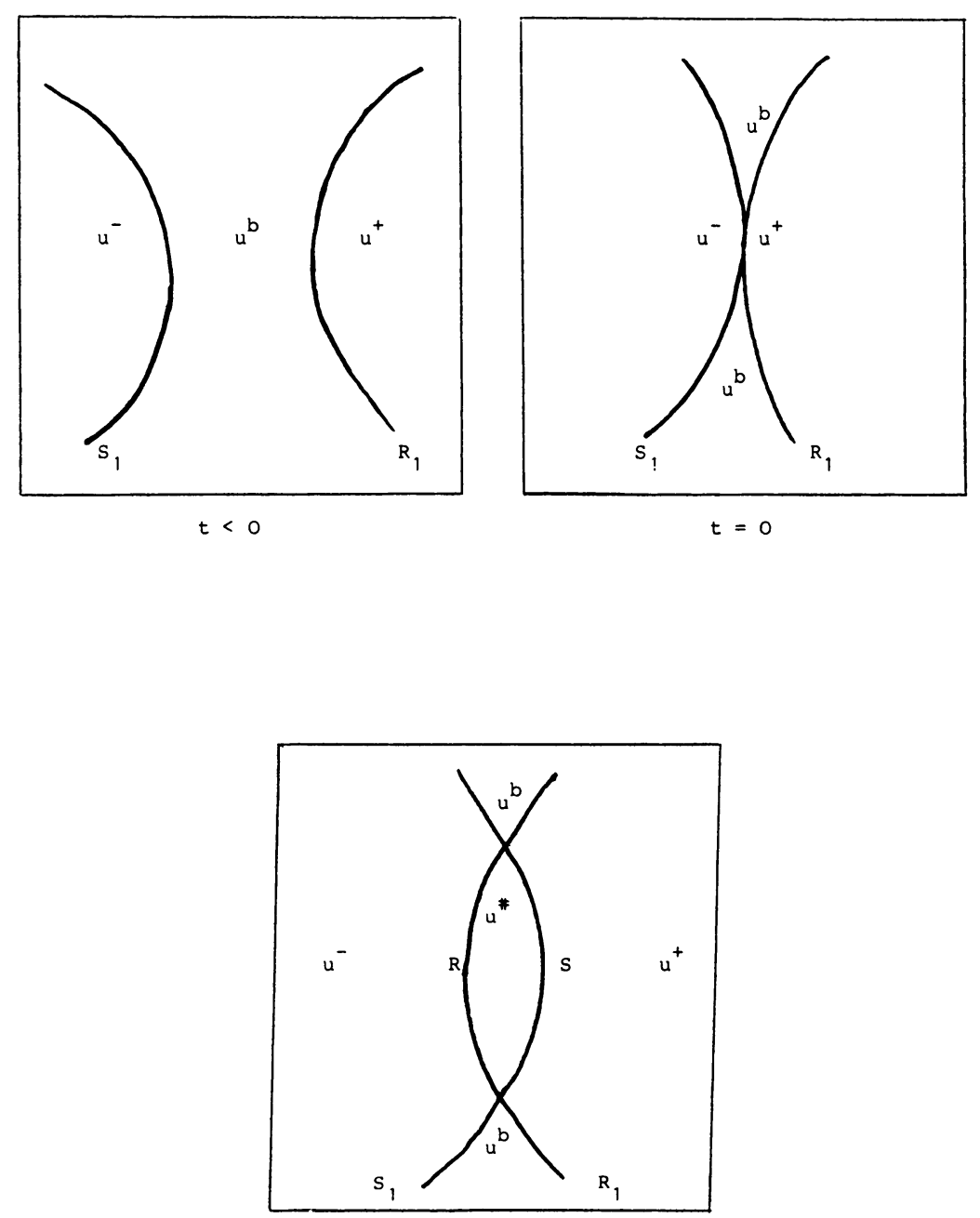

$t>0$

\section{Reduction à un problème de Goursat.}

3.1 Problème de Cauchy. Nous reprenons les notations du paragraphe 2.1 en supposant les hypothèses du Théorème 2.1.1 satisfaites: $\omega$ est un voisinage de l'origine dans le plan $(x, y), \varphi_{0}$ est une fonction $C^{\infty}$ sur un voisinage de 0 dans $\mathbf{R}$, $\omega_{ \pm}=\left\{(x, y) \in \omega \mid \pm\left(x-\varphi_{0}(y)\right)>0\right\}$, et $u_{0}^{ \pm}$sont les fonctions $C^{\infty}$ sur $\bar{\omega}_{ \pm}$. Nous noterons encore $u_{0}^{ \pm}$un prolongement $C^{\infty}$ à $\omega$, de ces fonctions. 
Le système (2.1.1) étant strictement hyperbolique, il existe au voisinage de l'origine dans $\mathbf{R}^{3}$ des fonctions $C^{\infty}, u^{+}(t, x, y)$ et $u^{-}(t, x, y)$, solutions de (2.1.1) et ayant pour données initiales:

$$
u_{\mid t=0}^{ \pm}=u_{0}^{ \pm}
$$

Pour démontrer le Théorème 2.1.3, il nous reste donc à construire des fonctions $\varphi$ et $\Psi$, vérifiant (2.1.3), et une fonction $u^{\#}$, définie et solution de (2.1.1), sur l'ouvert $\Omega_{\#}=\{\varphi(t, y)<x<\Psi(t, y)\}$, et satisfaisant aux conditions aux limites (2.1.5).

REMARQUe 3.1.1. La solution (2.1.4) ne dépend pas du choix des prolongements de $u_{0}^{ \pm}$qui déterminent $u^{ \pm}$; en effet, les conditions de choc (2.1.9) impliquent que $\rho<\lambda_{1}\left(\underline{u}^{-}\right)<\lambda_{2}\left(\underline{u}^{-}\right)$et $\sigma>\lambda_{2}\left(\underline{u}^{+}\right)>\lambda_{1}\left(\underline{u}^{+}\right)$, et la restriction de $u^{+}\left[\operatorname{resp} u^{-}\right.$] à $\Omega_{+}=\{x>\Psi(t, y)\}\left[\operatorname{resp} \Omega_{-}=\{x<\varphi(t, y)\}\right.$ ] ne dépend que de $u_{0 \mid \omega_{+}}^{+}$[resp. $u_{0 \mid \omega_{-}}^{-}$].

La construction de $\left(u^{\#}, \varphi, \Psi\right)$ est un problème à frontière libre, que nous ramenons en un problème sur un domaine fixe $\Omega=\left\{(\alpha, \beta, y) \in \mathbf{R}^{3} \mid \alpha>0, \beta>0\right\}$, à l'aide du changement de variables et de fonctions suivant

$$
u^{\#}(t, x, y)=v(\alpha, \beta, y)
$$

avec

$$
\alpha=t \frac{x-\varphi(t, y)}{\Psi(t, y)-\varphi(t, y)} \quad \beta=t \frac{\Psi(t, y)-x}{\Psi(t, y)-\varphi(t, y)} .
$$

Alors $\left(u^{\#}, \varphi, \Psi\right)$ sera solution de (2.1.1) (2.1.5) au voisinage de 0 dans $\bar{\Omega}_{\#}$ dès que $(v, \varphi, \Psi)$ sera solution au voisinage de 0 dans $\bar{\Omega}$ du problème suivant

$$
M \frac{\partial v}{\partial \alpha}+N \frac{\partial v}{\partial \beta}+B \frac{\partial v}{\partial y}=0
$$

$$
\left\{\begin{array}{c}
\left(v_{-}-\gamma^{\prime} v\right) \frac{\partial \varphi}{\partial t}+\left[g\left(v_{-}\right)-g\left(\gamma^{\prime} v\right)\right] \frac{\partial \varphi}{\partial y}+f\left(\gamma^{\prime} v\right)-f\left(v_{-}\right)=0 \\
\left(v_{+}-\gamma^{\prime \prime} v\right) \frac{\partial \Psi}{\partial t}+\left[g\left(v_{+}\right)-g\left(\gamma^{\prime \prime} v\right)\right] \frac{\partial \Psi}{\partial y}+f\left(\gamma^{\prime \prime}\right)-f\left(v_{+}\right)=0
\end{array}\right.
$$

$$
\left.\varphi\right|_{t=0}=\left.\Psi\right|_{t=0}=\varphi_{0},\left.\quad \frac{\partial \Psi}{\partial t}\right|_{t=0}>\left.\frac{\partial \varphi}{\partial t}\right|_{t=0},
$$

où l'on a noté

$$
\begin{aligned}
\gamma^{\prime} v(t, y)=v(0, t, y) & \gamma^{\prime \prime} v(t, y)=v(t, 0, y), \\
v_{-}(t, y)=u^{-}(t, \varphi(t, y), g) & v_{+}(t, y)=u^{+}(t, \Psi(t, y), y) .
\end{aligned}
$$

Les matrices $M, N$ et $B$ de (3.1.4) sont données par les formules

$$
\left\{\begin{array}{l}
M=\frac{\partial \alpha}{\partial t} \operatorname{Id}+\frac{\partial \alpha}{\partial x} A(v)+\frac{\partial \alpha}{\partial y} B(v) \\
N=\frac{\partial \beta}{\partial t} \operatorname{Id}+\frac{\partial \beta}{\partial x} A(v)+\frac{\partial \beta}{\partial y} B(v) \\
B=B(v)
\end{array}\right.
$$


avec, en notant $\theta(t, y)=t^{-1}(\Psi(t, y)-\varphi(t, y))$

$$
\left\{\begin{array}{l}
\frac{\partial \alpha}{\partial t}=-\left(\frac{1}{\theta} \frac{\partial \varphi}{\partial t}\right)(\alpha+\beta, y)-\alpha\left(\frac{1}{\theta} \frac{\partial \theta}{\partial t}\right)(\alpha+\beta, y), \\
\frac{\partial \alpha}{\partial x}=\left(\frac{1}{\theta}\right)(\alpha+\beta, y), \\
\frac{\partial \alpha}{\partial y}=-\left(\frac{1}{\theta} \frac{\partial \varphi}{\partial y}\right)(\alpha+\beta, y)-\alpha\left(\frac{1}{\theta} \frac{\partial \theta}{\partial y}\right)(\alpha+\beta, y),
\end{array}\right.
$$

et des formules analogues pour $\partial \beta / \partial t, \partial \beta / \partial x, \partial \beta / \partial y$.

On peut toujours supposer que $\underline{u}^{\#}=0$, ce que nous ferons par la suite; en notant $\phi=\left(\phi^{\prime}, \phi^{\prime \prime}\right)$ avec $\Phi^{\prime}=\varphi-\varphi_{0}-\rho t, \phi^{\prime \prime}=\Psi-\varphi_{0}-\sigma t$, et $\delta(t, y)=t^{-1}\left(\phi^{\prime \prime}-\phi^{\prime}\right)$ on a

LEMmE 3.1.1. Les matrices $M(v, \phi), N(v, \phi)$ et $B(0)$ sont des fonctions $C^{\infty}$ au voisinage de 0 dans $R^{12}$, des quantités: $y, v(\alpha, \beta, y), \nabla \phi(\alpha+\beta, y), \delta(\alpha+\beta, y)$, $\alpha(\nabla \delta)(\alpha+\beta, y)$ et $\beta(\nabla \delta)(\alpha+\beta, y)$. En outre, évaluées au point $0 \in \mathbf{R}^{12}$, ces fonctions valent respectivement

$$
\underline{M}=\frac{1}{\sigma-\rho}\left(A\left(\underline{u}^{\#}\right)-\rho \operatorname{Id}\right), \quad \underline{N}=\frac{1}{\sigma-\rho}\left(\sigma \operatorname{Id}-A\left(\underline{u}^{\#}\right)\right), \quad \underline{B}=B\left(\underline{u}^{\#}\right) .
$$

Dans ce lemme, comme par la suite, la notation $\nabla \phi$ désigne le gradient de la fonction $\phi$.

On peut réécrire les conditions aux limites (3.1.5) sous la forme

$$
\mathscr{F}^{\prime}\left(t, y, \gamma^{\prime} v, \nabla \phi^{\prime}, \phi^{\prime}\right)=0, \quad \mathscr{F}^{\prime \prime}\left(t, y, \gamma^{\prime \prime} v, \nabla \phi^{\prime \prime}, \phi^{\prime \prime}\right)=0,
$$

les fonctions $\mathscr{F}^{\prime}$ et $\mathscr{F}^{\prime \prime}$ étant des fonctions $C^{\infty}$ de leurs arguments. Leurs différentielles par rapport aux variables $\gamma v, \nabla \phi$ et $\phi$ seront notées

$$
\left\{\begin{array}{l}
F^{\prime}\left(\gamma^{\prime} w, \nabla \Psi^{\prime}, \Psi^{\prime}\right)=l^{\prime} \frac{\partial \Psi^{\prime}}{\partial t}+p^{\prime} \frac{\partial \Psi^{\prime}}{\partial y}+q^{\prime} \Psi^{\prime}+m^{\prime} \gamma^{\prime} w \\
F^{\prime \prime}\left(\gamma^{\prime \prime} w, \nabla \Psi^{\prime \prime}, \Psi^{\prime \prime}\right)=l^{\prime \prime} \frac{\partial \Psi^{\prime \prime}}{\partial t}+p^{\prime \prime} \frac{\partial \Psi^{\prime \prime}}{\partial y}+q^{\prime \prime} \Psi^{\prime \prime}+m^{\prime \prime} \gamma^{\prime \prime} w
\end{array}\right.
$$

LEMME 3.1.2. Les fonctions $l^{\prime}, p^{\prime}, q^{\prime}\left[\right.$ resp. $\left.m^{\prime}\right]$ sont des fonctions $C^{\infty}$ des variables $t, y, \gamma v, \nabla \phi$ et $\phi$, à valeurs dans $\mathbb{R}^{2}$ [resp. les matrices réelles $2 \times 2$ ]. En outre, pour la valeur 0 de leurs arguments, ces functions valent:

$$
\begin{array}{ll}
\underline{F}^{\prime}=0 ; & \underline{F}^{\prime \prime}=0, \\
\underline{l}^{\prime}=\underline{u}^{-}-\underline{u}^{\#} ; & \underline{l}^{\prime \prime}=\underline{u}^{+}-\underline{u}^{\#}, \\
\underline{p}^{\prime}=g\left(\underline{u}^{-}\right)-g\left(\underline{u}^{\#}\right) ; & \underline{p}^{\prime \prime}=g\left(\underline{u}^{+}\right)-g\left(\underline{u}^{\#}\right), \\
\underline{m}^{\prime}=A\left(\underline{u}^{\#}\right)-\rho \mathrm{Id} ; & \underline{\underline{m}}^{\prime \prime}=A\left(\underline{u}^{\#}\right)-\sigma \text { Id } .
\end{array}
$$

Nous énoncerons au paragraphe 3.3, un théorème permettant de résoudre des problèmes du type (3.1.4) (3.1.11); on achèvera ainsi la construction des fonctions $u^{\#}, \varphi$ et $\Psi$ et la démonstration du Théorème 2.1.3. 
3.2. Interaction de deux chocs. Nous nous plaçons maintenant sous les hypothèses du Théorème 2.2.2, et nous ramenons sa démonstration à la résolution d'un problème (3.1.4) (3.1.11).

Par hypothèse, les fonctions $u^{-}$et $u^{b}$ sont $C^{\infty}$ jusqu'en $t=0$; le travail de A. Majda [8] permet de résoudre le problème de Cauchy pour (2.1.1) avec donnée initiale:

$$
v_{0}(x, y)= \begin{cases}u^{-}(0, x, y) & \text { pour } x<\psi_{1}(0, y), \\ u^{b}(0, x, y) & \text { pour } x>\psi_{1}(0, y) .\end{cases}
$$

En effet, les conditions de compatibilité des données initiales sont automatiquement satisfaites, puisque ces conditions s'obtiennent par développement de Taylor autour de $t=0, x=\Psi_{1}(0, y)$ de l'équation (2.1.1) et de la condition de saut, et que $\left(u^{-}, u^{h}, \Psi_{1}\right)$ vérifient cette équation et cette condition de saut pour $t<0$. Bien que le théorème de [8] soit énoncé avec des hypothèses globales, notemment avec une surface de choc initiale compacte, la version locale que nous voulons utiliser ici, s'en déduit immédiatement. On obtient donc des fonctions, notées $u^{-}, v^{b}$ et $\Psi_{1}$, solutions pour $t>0$ assez petit de (2.1.1) et de la condition de saut de Rankine-Hugoniot. En fait, A. Majda montre l'existence d'une solution $H^{s}$, pour tout $s$ assez grand, sur un intervalle de temps qui dépend a priori de $s$; mais les inégalités d'énergie de [7 et 8], jointes aux méthodes habituelles de dérivation des équations et régularisation, et le fait que les conditions de compatibilité sont satisfaites à tout ordre, montrent que la solution est en fait $C^{\infty}$ sur un voisinage de 0 .

De même, on résoud le problème de Cauchy pour la donnée initiale:

$$
w_{0}(x, y)= \begin{cases}u^{b}(0, x, y) & \text { pour } x<\varphi_{1}(0, y), \\ u^{+}(0, x, y) & \text { pour } x>\varphi_{1}(0, y),\end{cases}
$$

et on obtient des fonctions $C^{\infty}$ au voisinage de 0 , notées $w^{b}, u^{+}$et $\varphi_{1}$, solutions de (2.1.1) et de la condition de saut.

Par hypothèse les surfaces $x=\Psi_{1}(t, y)$ et $x=\varphi_{1}(t, y)$ se coupent transversalement à l'origine et on peut paramétrer la courbe d'intersection sous la forme

$$
t=\tau(y), \quad x=\Psi_{1}(t, y)=\varphi_{1}(t, y)
$$

Notons $\Omega_{b}=\left\{(t, x, y) \mid t<\tau(y), \Psi_{1}(t, y)<x<\varphi_{1}(t, y)\right\}$. On a

LEMME 3.2.1. Les fonctions $v^{b}$ et $w^{b}$ coincident au voisinage de 0 , dans $\Omega_{b}$.

Preuve. $v^{b}$ et $w^{b}$ ont la même trace $u^{b}(0, x, y)$ sur l'ouvert $\omega_{b}=\{(x, y) \in$ $\left.\omega \mid \Psi_{1}(0, y)<x<\varphi_{1}(0, y)\right\}$, et prennent leurs valeurs dans un voisinage $V$ de $\underline{u}^{b}$, arbitrairement petit si on restreint le voisinage $\omega$ de 0 . Les conditions de choc pour (2.2.1) montrent que pour $t$ et $y$ voisins de 0 , et $u \in V$ on a

$$
\frac{\partial \varphi_{1}}{\partial t}(t, y)<\lambda_{1}(u)<\lambda_{2}(u)<\frac{\partial \Psi_{1}}{\partial t}(t, y)
$$

En restreignant $\omega$ si nécessaire, on voit alors que les surfaces $\Gamma_{\varepsilon}, \varepsilon \in[0,1]$ des points $(t, x, y)$ au dessus de $\omega_{b}$ tels que

$$
\begin{aligned}
& (1-\varepsilon)\left(x-\Psi_{1}(t, y)\right)\left(\varphi_{1}(t, y)-x\right) \\
& \quad+\varepsilon\left[\varphi_{1}(t, y)-\varphi_{1}(0, y)-\Psi_{1}(t, y)+\Psi_{1}(0, y)\right]=0
\end{aligned}
$$


sont non caratéristiques pour tout opérateur $\partial / \partial t+A(u)(\partial / \partial x)+B(u)(\partial / \partial y)$ avec $u \in V$. Coupant ces surfaces par un paraboloide $t \leqslant \varepsilon_{0}\left[\varepsilon_{1}^{2}-x^{2}-y^{2}\right]$, non caractéristique pour $\varepsilon_{0}$ assez petit, on obtient une famille de surfaces $\tilde{\Gamma}_{\varepsilon}$, s'appuyant sur le bord de $\tilde{\omega}_{b}=\left\{(x, y) \in \omega_{b} / x^{2}+y^{2} \leqslant \varepsilon_{1}^{2}\right\}$, et balayant, au voisinage de 0 , l'ouvert $\Omega_{b}$. Le Lemme 3.2.1 se déduit donc du théorèe d'unicité local pour les solutions régulières d'un système strictement hyperbolique.

Nous noterons $u^{b}$ la valeur commune de $v^{b}$ et $w^{b}$ sur $\Omega_{b}$, qui prolonge la fonction $u^{b}$ donnée dans $t<0$.

Pour achever la démonstration du Théorème 2.2.2, il nous reste à construire les fonctions $u^{\#}, \varphi$ et $\Psi$, vérifiant (2.2.6), telles que $u^{\#}$ soit solution de (2.1.1) sur l'ouvert $\Omega_{\#}=\{(t, x, y) / t>\tau(y), \varphi(t, y)<x<\Psi(t, y)\}$, et satisfaisant aux conditions de saut (2.1.5). Comme au paragraphe 3.1 on effectue un changement de variables:

$$
u^{\#}(t, x, y)=v(\alpha, \beta, y)
$$

avec

$$
\alpha=(t-\tau(y)) \frac{x-\varphi(t, y)}{\Psi(t, y)-\varphi(t, y)} \quad \beta=(t-\tau(y)) \frac{\Psi(t, y)-x}{\Psi(t, y)-\varphi(t, y)} .
$$

On peut supposer à nouveau que $\underline{u}^{\#}=0$ et on pose $\phi=\left(\phi^{\prime}, \phi^{\prime \prime}\right)$ avec

$$
\left\{\begin{array}{l}
\phi^{\prime}(t, y)=\varphi(t+\tau(y), y)-\varphi_{1}(\tau(y), y)-\rho t, \\
\phi^{\prime \prime}(t, y)=\Psi(t+\tau(y), y)-\Psi_{1}(\tau(y), y)-\sigma t,
\end{array}\right.
$$

On obtient à nouveau pour $(v, \phi)$, un problème du type suivant:

$$
\left\{\begin{array}{l}
M \frac{\partial v}{\partial \alpha}+N \frac{\partial v}{\partial \beta}+B \frac{\partial v}{\partial y}=0 \\
\mathscr{F}^{\prime}\left(t, y, \gamma^{\prime} v, \nabla \phi^{\prime}, \phi^{\prime}\right)=0 \\
\mathscr{F}^{\prime \prime}\left(t, y, \gamma^{\prime \prime} v, \nabla \phi^{\prime \prime}, \phi^{\prime \prime}\right)=0 \\
\left.\phi\right|_{t=0}=0
\end{array}\right.
$$

les Lemmes 3.1.1 et 3.1.2 étant encore exacts pour ce nouveau système.

3.3. Un problème de Goursat. Nous énonçons maintenant le théorème qui nous permettra de résoudre les problèmes (3.1.4) (3.1.11) et (3.2.9). Cependant il nous a paru commode de modifier légèrement les notations: nous noterons dorénavant $X=(x, y, z)$ la variable de $\mathbf{R}^{3}, \Omega$ le dièdre $x>0, y>0, \Omega_{T}$ sa section par $x+y<T$, et $\Omega_{T, R}$ l'ensemble $\left\{X \in \Omega_{t}|| z \mid<R\right\}$. De même $(t, z)$ désignant la variable de $\mathbf{R}^{2}$, $\omega$ sera le demi-plan $t>0, \omega_{T}$ la bande $0<t<T$, et $\omega_{T, R}$ le rectangle $] 0, T[x]-R, R[$. Si $\phi$ est une fonction définie sur $\omega$, on convient de noter $\tilde{\phi}$ la fonction définie sur $\Omega$ par

$$
\tilde{\phi}(x, y, z)=\phi(x+y, z) .
$$

Si $u$ est une fonction définie sur $\bar{\Omega}$, on note $\gamma u=\left(\gamma^{\prime} u, \gamma^{\prime \prime} u\right)$ ses traces:

$$
\gamma^{\prime} u(t, z)=u(0, t, z), \quad \gamma^{\prime \prime}(t, z)=u(t, 0, z) .
$$


Les inconnues étant $u$ et $\phi=\left(\phi^{\prime}, \phi^{\prime \prime}\right)$, fonctions définies respectivement sur $\Omega$ et $\omega$, à valeurs dans $\mathbf{R}^{2}$ on considère un système de la forme suivante:

$$
\left\{\begin{array}{l}
L(u, \phi) u \equiv A(u, \phi) \frac{\partial u}{\partial x}+B(u, \phi) \frac{\partial u}{\partial y}+C(u, \phi) \frac{\partial u}{\partial z}=0 \\
\mathscr{F}^{\prime}\left(t, z, \gamma^{\prime} u, \nabla \phi^{\prime}, \phi^{\prime}\right)=0 \\
\mathscr{F}^{\prime \prime}\left(t, z, \gamma^{\prime \prime} u, \nabla \phi^{\prime \prime}, \phi^{\prime \prime}\right)=0 \\
\phi_{\mid t=0}=0
\end{array}\right.
$$

où $\mathscr{F}^{\prime}$ et $\mathscr{F}^{\prime \prime}$ sont des fonctions $C^{\infty}$ de leurs arguments à valeurs dans $\mathbf{R}^{2}$; $A(u, \phi), B(u, \phi)$ et $C(u, \phi)$ sont des matrices $2 \times 2$ dont les coefficients sont des fonctions de la forme

$$
a(X, u(X), \tilde{\delta}(X), \widetilde{\nabla \phi}(X), x \widetilde{\nabla \delta}(X), y \widetilde{\nabla \delta}(X))
$$

avec $\delta(t, z)=t^{-1} \phi(t, z)$, et où a est une fonction $C^{\infty}$ de ses arguments au voisinage de 0 dans $\mathbf{R}^{15}$.

L'hypothèse de départ est la suivante:

(3.3.5) Les fonctions $\mathscr{F}^{\prime}$ et $\mathscr{F}^{\prime \prime}$ s'annulent à l'origine dans $\mathbf{R}^{7}$,

si bien que, si $\mathscr{F}^{\prime}$ et $\mathscr{F}^{\prime \prime}$ ne dépendaient pas de $(t, z), u=0, \phi=0$ serait solution triviale de (3.3.3). Il est alors naturel de linéariser ce système autour de cette solution et on pose

$$
\underline{L} v=\underline{A} \frac{\partial v}{\partial x}+\underline{B} \frac{\partial v}{\partial y}+\underline{C} \frac{\partial v}{\partial z}
$$

où $\underline{A}, \underline{B}, \underline{C}$ désignent les matrices obtenues en figeant les coefficients (3.3.4) en $0 \in \mathbf{R}^{15}$. De même, on note

$$
\left\{\begin{array}{l}
\underline{F}\left(\gamma^{\prime} v, \Psi^{\prime}\right)=\underline{l}^{\prime} \frac{\partial \Psi^{\prime}}{\partial t}+\underline{p}^{\prime} \frac{\partial \Psi^{\prime}}{\partial z}+\underline{q}^{\prime} \Psi^{\prime}+\underline{m}^{\prime} \gamma^{\prime} v \\
\underline{F}^{\prime \prime}\left(\gamma^{\prime \prime} v, \Psi^{\prime \prime}\right)=\underline{l}^{\prime \prime} \frac{\partial \Psi^{\prime \prime}}{\partial t}+\underline{p} \frac{\partial \Psi^{\prime \prime}}{\partial z}+\underline{q}^{\prime \prime} \Psi^{\prime \prime}+\underline{m}^{\prime \prime} \gamma^{\prime \prime} v
\end{array}\right.
$$

les différentielles à l'origine de $\mathscr{F}^{\prime}$ et $\mathscr{F}^{\prime \prime}$, par rapport aux variables $\gamma^{\prime} u, \nabla \phi^{\prime}, \phi^{\prime}$ et $\gamma^{\prime \prime} u, \nabla \phi^{\prime \prime}, \phi^{\prime \prime}$.

Pour condenser les notations on posera

$$
\mathscr{F}=\left(\mathscr{F}^{\prime}, \mathscr{F}^{\prime \prime}\right), \underline{F}(\gamma v, \Psi)=\left(\underline{F}\left(\gamma^{\prime} v, \Psi^{\prime}\right), \underline{F}^{\prime \prime}\left(\gamma^{\prime \prime} v, \Psi^{\prime \prime}\right)\right) \text {, etc. }
$$

Tout l'objet de ce travail est de montrer que, si le problème

$$
\underline{L} v=f, \quad \underline{F}(\gamma v, \Psi)=g,
$$

est suffisemment bien posé, alors le problème (3.3.3) possède effectivement une solution. Toutes nos hypothèses vont donc porter sur le problème linéaire à coefficients constants, (3.3.8). Elles seront de trois types:

( $\alpha$ ) Hypothèses $d$ 'hyperbolicite. On suppose $\underline{L}$ strictement hyperbolique dans la direction $(1,1,0)$; en outre, on suppose que les deux valeurs propres de $(\underline{A}+\underline{B})^{-1} \underline{A}$ vérifient

$$
a_{1}<0<1<a_{2}
$$


ReMARQUE. La condition (3.3.9) signifie que le problème de Goursat est raisonnable dans les ouverts $\Omega_{T}$ (cf. S. Alinhac [1]).

( $\beta$ ) Hypothèses de stabilité sur les faces du dièdre. Nous supposons que les matrices $\underline{m}^{\prime}$ et $\underline{m}^{\prime \prime}$ sont régulières et que les problèmes suivants vérifient la condition de Lopatinski uniforme (cf. A. Majda [7] et Appendice A).

$$
\begin{aligned}
& \left\{\begin{array}{l}
(\underline{A}+\underline{B}) \frac{\partial w}{\partial t}+\underline{A} \frac{\partial w}{\partial x}+\underline{C} \frac{\partial w}{\partial z}=f \quad \text { pour } x>0 \\
\underline{l}^{\prime} \frac{\partial \Psi^{\prime}}{\partial t}+\underline{p}^{\prime} \frac{\partial \Psi^{\prime}}{\partial z}+\underline{m}^{\prime} w_{\mid x=0}=g
\end{array}\right. \\
& \left\{\begin{array}{l}
(\underline{A}+\underline{B}) \frac{\partial w}{\partial t}+\underline{B} \frac{\partial w}{\partial y}+\underline{C} \frac{\partial w}{\partial z}=f \quad \text { pour } y>0 \\
l^{\prime \prime} \frac{\partial \Psi^{\prime \prime}}{\partial t}+\underline{p}^{\prime \prime} \frac{\partial \Psi^{\prime \prime}}{\partial z}+\underline{m}^{\prime \prime} w_{\mid y=0}=g
\end{array}\right.
\end{aligned}
$$

( $\gamma$ ) Hypothèses de stabilité sur l'arête du dièdre. Elles consistent à dire que le problème est bien posé au sens de séries de Taylor (cf. S. Alinhac [1]). Notant $r_{1}$ et $r_{2}$ des vecteurs propres de $(\underline{A}+\underline{B})^{-1} \underline{A}$ (cf. hypothèse $\left.\alpha\right)$ ), $b^{\prime}=\underline{m}^{\prime-1} \underline{l}^{\prime}, b^{\prime \prime}=\underline{m}^{\prime \prime-1} \underline{l}^{\prime \prime}$, et $\left(\alpha^{\prime}, \beta^{\prime}\right)$ et $\left(\alpha^{\prime \prime}, \beta^{\prime \prime}\right)$ les composantes de $b^{\prime}$ et $b^{\prime \prime}$ dans la base $\left(r_{1}, r_{2}\right)$, nous supposons que

$$
\left|\alpha^{\prime \prime} \beta^{\prime}\right|<\left|\alpha^{\prime} \beta^{\prime \prime}\right|
$$

ThEorème 3.3.1. Sous les hypothèses $(3.3 .5),(\alpha),(\beta)$ et $(\gamma)$ ci-dessus, il existe $T>0, R>0, u \in C^{1}\left(\bar{\Omega}_{T, R}\right)$ et $\phi \in C^{2}\left(\bar{\omega}_{T, R}\right)$ solution du problème (3.3.3). En outre $\nabla \phi(0,0)=0$.

Remarque 3.3.2 Les données étant $C^{\infty}$, on peut montrer que la solution $(u, \phi)$ est en fait $C^{\infty}$.

3.4. Fin de la preuve des Théorèmes 2.1.3 et 2.2.2. Les problèmes (3.1.4) (3.1.11) et (3.2.9) sont, aux notations près, du type (3.3.3); le Lemme 3.1.2 affirme que l'hypothèse (3.3.5) est satisfaite; les Lemmes 3.1.1 et 3.1.2 permettent d'identifier les quantités $\underline{A}, \underline{B}, \ldots, \underline{l}^{\prime}$, etc., en fonction de $\underline{u}^{+}, \underline{u}^{\#}, \ldots$, etc. les hypothèses $\left(\mathrm{H}_{1}\right)$, $\left(\mathrm{H}_{2}\right)$ et $\left(\mathrm{H}_{3}\right)$ assurent précisément que les hypothèses $(\alpha),(\beta)$ et $(\gamma)$ du Théorème 3.3.1 sont satisfaites. On peut donc résoudre les problèmes (3.1.4) (3.1.11) et (3.2.9), ce qui, compte tenu des paragraphes 3.1 et 3.2 , achève la démonstration des Théorèmes 2.1.3 et 2.2.2.

\section{Le problème sur un quadran.}

4.1. Notations et resultats. Pour $T>0$ on note $\Omega_{T}=\left\{(x, y) \in \mathbf{R}^{2} \mid x>0, y>0\right.$, $x+y<T\}$. On considère un système $2 \times 2$ du type

$$
L u \equiv A \frac{\partial u}{\partial x}+B \frac{\partial u}{\partial y}+C u=f
$$

où les matrices $A$ et $B$ [resp. $C$ ] sont de classe $C^{1}$ [resp. $C^{0}$ ] sur $\bar{\Omega}_{T_{0}}$. Pour $u$ définie $\operatorname{sur} \bar{\Omega}_{T}, \gamma u=\left(\gamma^{\prime} u, \gamma^{\prime \prime} u\right)$ désigne les traces $\gamma^{\prime} u(t)=u(0, t), \gamma^{\prime \prime} u(t)=u(t, 0)$. 
Le système (4.1.1) est couplé avec des conditions aux limites du type

$$
\left\{\begin{array}{l}
F^{\prime}\left(\gamma^{\prime} u, \phi^{\prime}\right) \equiv l^{\prime} \frac{d \phi^{\prime}}{d t}+g^{\prime} \phi^{\prime}+m^{\prime} \gamma^{\prime} u=g^{\prime} \\
F^{\prime \prime}\left(\gamma^{\prime \prime} u, \phi^{\prime \prime}\right) \equiv l^{\prime \prime} \frac{d \phi^{\prime \prime}}{d t}+q^{\prime \prime} \phi^{\prime \prime}+m^{\prime \prime} \gamma^{\prime \prime} u=g^{\prime \prime} \\
\phi^{\prime}(0)=\phi^{\prime \prime}(0)=0
\end{array}\right.
$$

Les vecteurs $l^{\prime}, l^{\prime \prime}, q^{\prime}, q^{\prime \prime}$ et les matrices $m^{\prime}, m^{\prime \prime}$ étant continus sur [0, $\left.T_{0}\right]$. Nous noterons $\phi=\left(\phi^{\prime}, \phi^{\prime \prime}\right)$ et $F(\gamma u, \phi)=\left(F^{\prime}\left(\gamma^{\prime} u, \phi^{\prime}\right), F^{\prime \prime}\left(\gamma^{\prime \prime} u, \phi^{\prime \prime}\right)\right)$.

On se donne aussi un système $(\underline{L}, \underline{F})$ comme en (4.1.1), (4.1.2), à coefficients $\underline{A}$, $\underline{B}, \underline{l}, \underline{m}$ constants, avec $\underline{C}=0, \underline{q}=0$, l'idée étant de considérer $(L, F)$ comme une perturbation de $(\underline{L}, \underline{F})$; en particulier nous utiliserons la notation suivante:

$$
\begin{aligned}
\varepsilon(L, F)=\operatorname{Max}\left\{\|A-\underline{A}\|_{L^{\infty}\left(\Omega_{T_{0}}\right)},\|B-\underline{B}\|_{L^{\infty}\left(\Omega_{T_{0}}\right)},\right. \\
\\
\left.\|l-\underline{l}\|_{L^{\infty}\left(\left[0, T_{0}\right]\right)},\|m-\underline{m}\|_{L^{\infty}\left(\left[0, t_{0}\right]\right)}\right\} .
\end{aligned}
$$

Nous ferons, sur le système $(\underline{L}, \underline{F})$, l'hypothèse suivante:

(i) La matrice $(\underline{A}+\underline{B})^{-1} \underline{A}$ a deux valeurs propres réelles qui vérifient:

(ii) Le système $(\underline{L}, \underline{F})$ satisfait à l'hypothèse $(\gamma)$ du paragraphe 3.3.

Avant d'énoncer le résultat introduisons une dernière notation: pour $\lambda \geqslant 0$, $C_{\lambda}^{k}\left(\bar{\Omega}_{T}\right)$ [resp. $\left.C_{\lambda}^{k}([0, T])\right]$ désigne l'ensemble des fonctions $u$ de classe $C^{k}$ sur $\bar{\Omega}_{T}$ [resp. $h$ de classe $C^{k}$ sur $[0, T]$ telles que $(x+y)^{-\lambda} u(x, y)$ [resp. $t^{-\lambda} h(t)$ ] soit bornée.

ThEORÈme 4.1.1. Sous l'hypothèse $(\mathrm{H})$ ci-dessus, il existe $\varepsilon_{0}>0$ tel que si l'on a $\varepsilon(L, F) \leqslant \varepsilon_{0}$ alors, pour $T \leqslant T_{0}$, on a:

(i) Pour tour $f \in C^{0}\left(\bar{\Omega}_{T}\right)$, tout $g \in C^{0}([0, T])$, il existe une unique solution $(u, \phi)$ $\in C^{0}\left(\bar{\Omega}_{T}\right) \times C^{1}([0, T])$ du problème

$$
L u=f, \quad F(\gamma u, \phi)=g, \quad \phi(0)=0 .
$$

(ii) Si $f \in C_{\lambda-1}^{0}\left(\bar{\Omega}_{T}\right), g \in C_{\lambda}^{0}([0, T])$ pour un $\lambda \geqslant 1$, la solution $(u, \phi)$ est dans l'espace $C_{\lambda}^{0}\left(\bar{\Omega}_{T}\right) \times C_{\lambda+1}^{1}([0, T])$.

(iii) Si les coefficients de $L$ et $f$ sont $C^{\infty}$ sur $\bar{\Omega}_{T}$ et si les coefficients de $F$ et $g$ sont $C^{\infty}$ sur $[0, T]$, alors la solution $(u, \phi)$ est dans l'espace $C^{\infty}\left(\bar{\Omega}_{T}\right) \times C^{\infty}([0, T])$.

La démonstration de ce théorème s'inspire directement du travail de $\mathrm{S}$. Alinhac. Nous étudions d'abord un cas particulier (\$4.2), avant de ramener le cas général à ce cas particulier (\$4.3).

4.2. Un cas particulier. Considérons un système de la forme

$$
L u=A \frac{\partial u}{\partial x}+B \frac{\partial u}{\partial y}
$$




$$
\left\{\begin{array}{l}
F^{\prime}\left(\gamma^{\prime} u, \phi^{\prime}\right)=l^{\prime} \frac{d \phi^{\prime}}{d t}+\gamma^{\prime} u, \\
F^{\prime \prime}\left(\gamma^{\prime \prime} u, \phi^{\prime \prime}\right)=l^{\prime \prime} \frac{d \phi^{\prime \prime}}{d t}+\gamma^{\prime \prime} u,
\end{array}\right.
$$

où $A$ est une matrice diagonale,

$$
A=\left(\begin{array}{cc}
a_{1} & 0 \\
0 & a_{2}
\end{array}\right) \text { et } B=I-A .
$$

On suppose que $A$ est de classe $C^{1}$ sur $\bar{\Omega}_{T_{0}}$ et que

$$
a_{1}<0<1<a_{2} \quad \operatorname{sur} \bar{\Omega}_{T_{0}} .
$$

Les vecteurs $l^{\prime}=\left(\begin{array}{c}\alpha^{\prime} \\ \beta^{\prime}\end{array}\right)$ et $l^{\prime \prime}=\left(\begin{array}{c}\alpha^{\prime \prime} \\ \beta^{\prime \prime}\end{array}\right)$ sont supposés continus sur $\left[0, T_{0}\right]$, et vérifient

$$
\left|\alpha^{\prime \prime} \beta^{\prime}\right|<\left|\alpha^{\prime} \beta^{\prime \prime}\right| \text { sur }\left[0, T_{0}\right] .
$$

Nous munissons les espaces $C^{0}\left(\bar{\Omega}_{T}\right)$ [resp. $\left.C^{0}([0, T])\right]$ des normes suivantes:

$$
\|u\|_{\tau}=\left\|e^{-\tau(x+y)} u\right\|_{L^{\infty}\left(\Omega_{T}\right)} \quad\left[\text { resp. }\|h\|_{\tau}=\left\|e^{-\tau t} h\right\|_{L^{\infty}([0, T)]}\right] .
$$

Proposition 4.2.1. Il existe $C$ tel que pour tous $T \leqslant T_{0}, \tau>0, f \in C^{0}\left(\bar{\Omega}_{T}\right)$ et $g \in C^{0}([0, T])$, le problème

$$
L u=f, \quad F(\gamma u, \phi)=g, \phi(0)=0,
$$

possède une unique solution $(u, \phi) \in C^{0}\left(\bar{\Omega}_{T}\right) \times C^{1}([0, T])$ qui vérifie

$$
\|u\|_{\tau}+\left\|\frac{d \phi}{d t}\right\|_{\tau}+\tau\|\phi\|_{\tau} \leqslant C\left\{\frac{1}{\tau}\|f\|_{\tau}+\|g\|_{\tau}\right\}
$$

La démonstration se fait en plusieurs étapes

(1) Découplage des conditions aux limites.

Notons

$$
u=\left(\begin{array}{l}
u_{1} \\
u_{2}
\end{array}\right), \quad g^{\prime}=\left(\begin{array}{l}
g_{1}^{\prime} \\
g_{2}^{\prime}
\end{array}\right), \quad g^{\prime \prime}=\left(\begin{array}{l}
g_{1}^{\prime \prime} \\
g_{2}^{\prime \prime}
\end{array}\right)
$$

et posons $\eta^{\prime}=\beta^{\prime} / \alpha^{\prime}, \eta^{\prime \prime}=\alpha^{\prime \prime} / \beta^{\prime \prime}$. L'équation $F(\gamma u, \phi)=g$ se découple immédiatement sous la forme

$$
\begin{gathered}
\left\{\begin{array}{l}
\gamma^{\prime} u_{2}=\eta^{\prime} \gamma^{\prime} u_{1}+\left(g_{2}^{\prime}-\eta^{\prime} g_{1}^{\prime}\right)=\eta^{\prime} \gamma^{\prime} u_{1}+h_{1}, \\
\gamma^{\prime \prime} u_{1}=\eta^{\prime \prime} \gamma^{\prime \prime} u_{2}+\left(g_{1}^{\prime \prime}-\eta^{\prime \prime} g_{2}^{\prime \prime}\right)=\eta^{\prime \prime} \gamma^{\prime \prime} u_{2}+h_{2},
\end{array}\right. \\
\begin{cases}\frac{d \phi^{\prime}}{d t}=\frac{1}{\alpha^{\prime}}\left(g_{1}^{\prime}-\gamma^{\prime} u_{1}\right), & \phi^{\prime}(0)=0, \\
\frac{d \phi^{\prime \prime}}{d t}=\frac{1}{\beta^{\prime \prime}}\left(g_{2}^{\prime \prime}-\gamma^{\prime \prime} u_{2}\right), & \phi^{\prime \prime}(0)=0,\end{cases}
\end{gathered}
$$


(2) Reduction à une équation fonctionnelle. Notons

$$
b_{1}=\frac{a_{1}}{a_{1}-1}, \quad b_{2}=\frac{a_{2}-1}{a_{2}}, \quad f=\left(\begin{array}{c}
\left(1-a_{1}\right) \cdot f_{1} \\
a_{2} \cdot f_{2}
\end{array}\right) .
$$

Alors l'équation $L u=f$ s'écrit

$$
-b_{1} \frac{\partial u_{1}}{\partial x}+\frac{\partial u_{1}}{\partial y}=f_{1}, \quad \frac{\partial u_{2}}{\partial x}-b_{2} \frac{\partial u_{2}}{\partial y}=f_{2} .
$$

Par (4.2.3), on a $0<b_{i}<1$ pour $i=1$, 2. Par conséquent, la caractéristique de $-b_{1} \partial x+\partial y$ [resp. $\partial x-b_{2} \partial y$ ] issue d'un point $\left(x_{0}, y_{0}\right) \in \Omega_{T}$, est, pour $0<y<y_{0}$ [resp. $0<x<x_{0}$ ], contenue dans $\Omega_{T}$, et coupe l'axe $y=0$ [resp. $x=0$ ] en un point d'abscisse $X\left(x_{0}, y_{0}\right)$ [resp. d'ordonnée $Y\left(x_{0}, y_{0}\right)$ ]. En outre, les fonctions $(x, y) \rightarrow$ $X(x, y)$ et $(x, y) \rightarrow Y(x, y)$ sont de classe $C^{1}$ sur $\Omega_{T_{0}}$, et vérifient

$$
x \leqslant X(x, y) \leqslant x+y \quad y \leqslant Y(x, y) \leqslant x+y .
$$

Toute solution continue de (4.2.9) est donc de la forme

$$
\begin{aligned}
& u_{1}(x, y)=\gamma^{\prime \prime} u_{1}(X(x, y))+F_{1}(x, y), \\
& u_{2}(x, y)=\gamma^{\prime} u_{2}(Y(x, y))+F_{2}(x, y),
\end{aligned}
$$

où $F_{1}(x, y)$ [resp. $F_{2}(x, y)$ ] est l'intégrale de $f_{1}$ [resp. $f_{2}$ ] le long de l'arc de caractéristique joignant $(X(x, y), 0)$ à $(x, y)$ [resp. $(0, Y(x, y))$ à $(x, y)]$.

(4.2.11) conduit aux relations

$$
\begin{aligned}
& \gamma^{\prime} u_{1}(t)=\gamma^{\prime \prime} u_{1}(X(0, t))+F_{1}(0, t), \\
& \gamma^{\prime \prime} u_{2}(t)=\gamma^{\prime} u_{2}(Y(t, 0))+F_{2}(t, 0),
\end{aligned}
$$

et la comparaison avec (4.2.7) conduit a l'équation

$$
\gamma^{\prime \prime} u_{1}(t)=\eta^{\prime} \eta^{\prime \prime}(t) \gamma^{\prime \prime} u_{1}(Z(t))+h(t)
$$

avec $Z(t)=X(0, Y(t, 0))$, et

$$
h(t)=h_{2}(t)+\eta^{\prime \prime}(t) h_{1}(Y(t, 0))+\eta^{\prime \prime}(t) F_{2}(t, 0)+\eta^{\prime}(t) \eta^{\prime \prime}(t) F_{1}(0, Y(t, 0)) .
$$

(3) Résolution de (4.2.13).

Lemme 4.2.2. Il existe $C$ tel que pour $T \leqslant T_{0}, \tau>0, f \in C^{0}\left(\bar{\Omega}_{T}\right)$, on ait $F=$ $\left(F_{1}, F_{2}\right) \in C^{0}\left(\bar{\Omega}_{T}\right)$ et $\|F\|_{\tau} \leqslant(C / \tau)\|f\|_{\tau}$.

Preuve. Notant $(x(s), s)$ l'arc de caractéristique joignant $(X(x, y), 0)$ à $(x, y)$ on a

$$
F_{1}(x, y)=\int_{0}^{y} f_{1}(x(s), s) d s,
$$

Par ailleurs $(d / d s)(x(s)+s)=1-b_{1}(x(s), s) \geqslant k>0$ et par conséquent

$$
s+x(s) \leqslant x+y-k(y-s) \text {. }
$$


On en déduit la majoration

$$
\left|e^{-\tau(x+y)} F_{1}(x, y)\right| \leqslant C\|f\|_{\tau} \int_{0}^{y} e^{-\tau k(y-s)} d s .
$$

$F_{2}$ vérifie une estimation semblable et le lemme suit.

Lemme 4.2.3. Il existe $\rho<1$ tel que pour tout $T \leqslant T_{0}, \tau>0$, l'opérateur $v \rightarrow \Lambda v$ $=\eta^{\prime} \eta^{\prime \prime} . v \circ Z$ opère de $C^{0}([0, T])$ dans lui-même avec $\|\Lambda v\|_{\tau} \leqslant \rho\|v\|_{\tau}$.

Preuve. $Z$ est une application continue de $\left[0, T_{0}\right]$ dans lui-même et, par (4.2.10), $Z(t) \leqslant t$. Par ailleurs l'hypothèse (4.2.4) signifie que $\rho=\left\|\eta^{\prime} \eta^{\prime \prime}\right\|_{L^{\infty}\left(T_{0}\right)}<1$. Compte tenu de la définition (4.2.5) des normes, le lemme en résulte immédiatement.

Corollaire 4.2.4. Il existe $C$ tel que pour tout $T \leqslant T_{0}, \tau>0, h \in C^{0}([0, T])$, l'équation $v-\Lambda v=h$ possède une unique solution dans $C^{0}([0, T])$ qui vérifie $\|v\|_{\tau} \leqslant$ $c\|h\|_{\tau}$.

(4) Démonstration de la Proposition 4.2.1. Soit $(f, g) \in C^{0}\left(\bar{\Omega}_{T}\right) \times C^{0}([0, T])$. Les fonctions $t \rightarrow X(0, t)$ et $t \rightarrow Y(t, 0)$ sont continues et vérifient $X(0, t) \leqslant t, Y(t, 0) \leqslant$ $t$. Il résulte du Lemme 4.2.2 et de la définition (4.2.5), que la fonction $h,(4.2 .14)$ est dans $C^{0}([0, T])$ et vérifie

$$
\|h\|_{\tau} \leqslant C\left\{(1 / \tau)\|f\|_{\tau}+\|g\|_{\tau}\right\} .
$$

Le corollaire (4.2.4) fournit une solution $v$ à l'équation

$$
v=\eta \eta^{\prime} v \circ Z+h \text {. }
$$

On définit alors successivement: $\tilde{v}=v \circ X ; u_{1}=\tilde{v}+F_{1} ; w=\eta^{\prime} \gamma^{\prime} u_{1}+h_{1} ; \tilde{w}=$ $w \circ Y$ et $u_{2}=\tilde{w}+F_{2}$.

Par construction, $\gamma^{\prime \prime} u_{1}=v$ et $\gamma^{\prime} u_{2}=w ; u_{1}$ et $u_{2}$ sont solutions de (4.2.9); la première condition aux limites de (4.2.7) résulte du choix de $w$, quant à la seconde, elle résulte du fait que $v=\gamma^{\prime \prime} u_{1}$ est solution de (4.2.18). Enfin, en utilisant (4.2.10) on a les estimations suivantes:

$$
\begin{gathered}
\|\tilde{v}\|_{\tau} \leqslant\|v\|_{\tau}, \quad\left\|u_{1}\right\|_{\tau} \leqslant\|\tilde{v}\|_{\tau}+\left\|F_{1}\right\|_{\tau}, \\
\|w\|_{\tau} \leqslant C\left\{\left\|u_{1}\right\|_{\tau}+\|g\|_{\tau}\right\} \quad\|\tilde{w}\|_{\tau} \leqslant\|w\|_{\tau}, \\
\left\|u_{2}\right\|_{\tau} \leqslant\|\tilde{w}\|_{\tau}+\left\|F_{2}\right\|_{\tau} .
\end{gathered}
$$

Avec (4.2.17), le Corollaire (4.2.4), et le Lemme 4.2.2, on obtient finalement une solution u de (4.2.9) (4.2.7) qui vérifie

$$
\|u\|_{\tau} \leqslant C\left\{\tau^{-1}\|f\|_{\tau}+\|g\|_{\tau}\right\}
$$

Maintenant $\phi$ est déterminé par (4.2.8) et on a

$$
\tau\|\phi\|_{\tau} \leqslant\|d \phi / d t\|_{\tau} \leqslant C\left\{\|g\|_{\tau}+\|u\|_{\tau}\right\} \text {. }
$$

Il reste maintenant à vérifier l'unicité de la solution de (4.1.4): elle résulte clairement de l'analyse faite à la 2ème étape et de l'unicité de la solution de (4.2.13).

Nous munissons maintenant les espaces $C_{\lambda}^{0}\left(\bar{\Omega}_{T}\right)$ [resp. $\left.C_{\lambda}^{0}([0, T])\right]$ des normes suivantes:

$$
\|u\|_{\tau, \lambda}=\left\|(x+y)^{-\lambda} e^{-\tau(x+y)} u\right\|_{L^{\infty}\left(\Omega_{T}\right)}
$$

[resp. $\left.|h|_{\tau, \lambda}=\left|t^{-\lambda} e^{-\tau t} h\right|_{L^{\infty}([0, T])}\right]$. 
Pour $\lambda \geqslant 1$ on munit aussi $C_{\lambda-1}^{0}\left(\bar{\Omega}_{T}\right)$ de la norme

$$
\|f\|_{\tau, \lambda}^{\prime}=\left\|\left(\lambda(x+y)^{\lambda-1}+\tau(x+y)^{\lambda}\right)^{-1} e^{-\tau(x+y)} f\right\|_{L^{\infty}\left(\Omega_{T}\right)} .
$$

Nous introduisons l'espace $\tilde{C}_{\lambda+1}^{1}([0, T])$ des fonctions $\phi \in C_{\lambda+1}^{0}([0, T]) \cap$ $C^{1}([0, T])$ telle que $\phi(0)=0$ et $d \phi / d t \in C_{\lambda}^{0}([0, T])$. Cet espace est muni des normes $\|d \phi / d t\|_{\tau, \lambda}$ en remarquant que pour $\phi \in \tilde{C}_{\lambda+1}^{1}([0, T])$ on a

$$
(\lambda+1)\|\phi\|_{\tau, \lambda+1}+\tau\|\phi\|_{\tau, \lambda} \leqslant 2\|d \phi / d t\|_{\tau, \lambda} .
$$

Proposition 4.2.5. Il existe $C>0$ tel que, pour $T \leqslant T_{0}, \lambda \geqslant 1, \tau>0, f \in$ $C_{\lambda-1}^{0}\left(\bar{\Omega}_{T}\right), \quad g \in C_{\lambda}^{0}([0, T])$, la solution $(u, \phi)$ de (4.1.4) soit dans $C_{\lambda}^{0}\left(\bar{\Omega}_{T}\right) \times$ $\tilde{C}_{\lambda+1}^{1}([0, T])$ et vérifie

$$
\|u\|_{\tau, \lambda}+\|d \phi / d t\|_{\tau, \lambda} \leqslant C\left\{\|f\|_{\tau, \lambda}^{\prime}+\|g\|_{\tau, \lambda}\right\} .
$$

Preuve. On reprend la démonstration de la Proposition 4.2.1. L'estimation du Lemme 4.2.2 est à remplacer par $\|F\|_{\tau, \lambda} \leqslant C\|f\|_{\tau, \lambda}^{\prime}$ comme cela résulte immédiatement de (4.2.15) (4.2.16) et de la définition (4.2.20). On a à nouveau

$$
\left\|\eta^{\prime} \eta^{\prime \prime}(v \circ Z)\right\|_{\tau, \lambda} \leqslant \rho\|v\|_{\tau, \lambda}
$$

et on conclut, suivant les lignes de la preuve de la Proposition 4.2.1, que

$$
\|u\|_{\tau, \lambda} \leqslant C\left\{\|f\|_{\tau, \lambda}^{\prime}+\|g\|_{\tau, \lambda}\right\} .
$$

L'estimation de $\phi$ découle trivialement de (4.2.8).

Pour finir, nous étudions la régularité des solutions de (4.1.4). Munisons les espaces $C^{k}\left(\bar{\Omega}_{T}\right)$ [resp. $\left.C^{k}([0, T])\right]$ des normes suivantes

$$
\|u\|_{k, \tau}=\sum_{|\alpha| \leqslant k} \tau^{k-|\alpha|}\left\|\partial^{\alpha} u\right\|_{\tau}
$$

[resp. $\left.\||h|\|_{k, \tau}=\sum_{j=0}^{k} \tau^{k-j}\left\|\partial_{t}^{j} h\right\|_{\tau}\right]$.

En supposant les coefficients de $L$ et $F, C^{\infty}$ sur $\bar{\Omega}_{T_{0}}$ et $\left[0, T_{0}\right]$, on a le résultat suivant

Proposition 4.2.6. Pour tout entier $k$ il existe $C$ et $\tau_{0}$ tels que pour tous $T \leqslant T_{0}$, $\tau \geqslant \tau_{0}, f \in C^{k}\left(\bar{\Omega}_{T}\right)$ et $g \in C^{k}([0, T])$ la solution $(u, \phi)$ de (4.1.4) soit dans $C^{k}\left(\bar{\Omega}_{T}\right)$ $\times C^{k+1}([0, T])$ et vérifie

$$
\|u\|_{k, \tau}+\|\phi \mid\|_{k+1, \tau} \leqslant C\left\{\tau^{-1}\|f\|_{k, \tau}+\|g\|_{k, \tau}\right\} .
$$

Preuve. On reprend la démonstration de la Proposition 4.2.1. Les applications $X(x, y)$ et $Y(x, y)$ sont maintenant $C^{\infty}$ sur $\bar{\Omega}_{T_{0}}$; l'estimation du Lemme 4.2.2 est à remplacer par $\left\|F\left|\left\|_{k, \tau} \leqslant(C / \tau)\right\| f\right|\right\|_{k, \tau}$ et l'estimation (4.2.17) par

$$
\|h\|_{k, \tau} \leqslant C\left\{\tau^{-1}\|f\|_{k, \tau}+\|g\|_{k, \tau}\right\} .
$$

Maintenant, puisque $\eta^{\prime} \eta^{\prime \prime} \in C^{\infty}\left(\left[0, T_{0}\right]\right)$ avec $\rho=\left\|\eta^{\prime} \eta^{\prime \prime}\right\|_{L^{\infty}}<1$, et que $Z(t) \in$ $C^{\infty}\left(\left[0, T_{0}\right]\right)$ avec $Z(t) \leqslant t$, on voit que pour $\rho<\rho^{\prime}<1$ et $\tau$ assez grand on a:

$$
\|\| \eta^{\prime} \eta^{\prime \prime} \cdot v \circ Z \mid\left\|_{k, \tau} \leqslant \rho^{\prime}\right\| v \|_{k, \tau} .
$$


On en déduit que la solution $v$ de (4.2.18) est dans $C^{k}([0, T])$ et vérifie pour $\tau$ assez grand

$$
\|v \mid\|_{k, \tau} \leqslant c\|\| h \|_{k, \tau}
$$

Les formules explicites donnant $\mathrm{u}$ et $\phi$ à partir de $v, F$ et $g$ données dans la démonstration de la Proposition 4.2.1, montrent que $(u, \phi) \in C^{k}\left(\bar{\Omega}_{T}\right) \times$ $C^{k+1}([0, T])$, avec l'estimation annoncée.

4.3. Démonstration du Théorème 4.1.1. Soit $(L, F)$ un système (4.1.1) (4.1.2) vérifiant (H). Si $\varepsilon(L, F)$ est assez petit, $(A+B)^{-1} A$ a, pour $(x,) \in \bar{\Omega}_{T_{0}}$, deux valeurs propres $a_{1}$ et $a_{2}$ qui vérifient (4.2.3). Notant $S$ une matrice de diagonalisation de $(A+B)^{-1} A$, de classe $C^{1}$ (ou $C^{\infty}$ si $A$ et $B$ le sont), en effectuant le changement de fonctions $u=S^{-1} \tilde{f}, f=S^{-1}(A+B) \tilde{f}$, on se ramène au cas où $A$ est diagonale, $B=I-A$, avec la propriété (4.2.3). Pour $\varepsilon(L, F)$ assez petit, les matrices $m^{\prime}$ et $m^{\prime \prime}$ sont inversibles sur $\left[0, T_{0}\right]$ et on se ramène immédiatement au cas où $m^{\prime}=m^{\prime \prime}=\mathrm{Id}$. On a donc

$$
L u=L_{0} u+C u, \quad F(\gamma u, \phi)=F_{0}(\gamma u, \phi)+q \phi,
$$

avec $\left(L_{0}, F_{0}\right)$ de la forme (4.2.1) (4.2.2), vérifiant (4.2.3) et (4.2.4).

Le Théorème 4.1.1 résulte alors des Propositions 4.2.1, 4.2.5 et 4.2.6 et des estimations suivantes, qui découlent des définitions (4.2.5), (4.2.19), (4.2.20) et (4.2.22)

$$
\begin{gathered}
\begin{cases}\|C u\|_{\tau} \leqslant K\|u\|_{\tau} & \text { pour } u \in C^{0}\left(\bar{\Omega}_{T}\right), \\
\|q \phi\|_{\tau} \leqslant K\|\phi\|_{\tau} & \text { pour } \phi \in C^{0}([0, T]),\end{cases} \\
\begin{cases}\|C u\|_{\tau, \lambda}^{\prime} \leqslant(K / \tau)\|u\|_{\tau, \lambda} & \text { pour } u \in C_{\lambda}^{0}\left(\bar{\Omega}_{T}\right), \\
\|q \phi\|_{\tau, \lambda} \leqslant(K / \tau)\|d \phi / d t\|_{\tau, \lambda} & \text { pour } \phi \in \tilde{C}_{\lambda+1}^{1}\left(\bar{\Omega}_{T}\right),\end{cases}
\end{gathered}
$$

et, si $C$ et $q$ sont $C^{\infty}$

$$
\begin{cases}\|C u\|_{k, \tau} \leqslant K\|\| u \|_{k, \tau} & \text { pour } u \in C^{k}\left(\bar{\Omega}_{T}\right), \\ \|g \phi\|_{k, \tau} \leqslant(K / \tau)\|\phi\|_{k+1, \tau} & \text { pour } \phi \in C^{k+1}([0, T]) .\end{cases}
$$

5. Espaces à poids. Nous regroupons dans cette partie un certain nombre de résultats techniques que nous utiliserons par la suite.

5.1. Notations et définitions. Nous revenons aux notations du paragraphe 3.3: $\Omega$ désigne le dièdre $\left\{(x, y, z) \in \mathbf{R}^{3} \mid x>0, y>0\right\}$ et $\Omega_{T}$ sa section par $x+y<T$. De même $\omega=\left\{(t, z) \in \mathbf{R}^{2} \mid t>0\right\}$ et $\omega_{T}$ est la bande $0<t<T$.

Pour $k$ entier $H^{k}(\cdot)$ désigne l'espace de Sobolev usuel muni de sa norme naturelle. Pour $\lambda$ réel $L_{\lambda}^{2}\left(\Omega_{T}\right)$ [resp. $L_{\lambda}^{2}\left(\omega_{T}\right)$ ] est l'espace des $u$ tels que $(x+y)^{-\lambda} u \in L^{2}\left(\Omega_{T}\right)$ [resp. $t^{-\lambda} u \in L^{2}\left(\omega_{T}\right)$ ]. Pour $k \in \mathbf{N}, H_{\lambda}^{k}\left(\Omega_{T}\right)$ [resp. $H_{\lambda}^{k}\left(\omega_{T}\right)$ ] est l'espace des $u$ tels que $\partial^{\alpha} u \in L_{\lambda-|\alpha|}^{2}$ pour toute dérivation de longueur $|\alpha| \leqslant k$.

Ces espaces sont munis des normes suivantes:

$$
\|u\|_{k, \lambda, T}=\left\{\sum_{|\alpha| \leqslant k}(1+|\lambda|)^{2(k-|\alpha|)}\left\|\partial^{\alpha} u\right\|_{L_{\lambda-|\alpha|}^{2}}^{2}\right\}^{1 / 2}
$$


On introduit enfin les espaces $\dot{C}^{\infty}\left(\Omega_{T}\right)$ [resp. $\dot{C}^{\infty}\left(\omega_{T}\right)$ ] des fonctions $C^{\infty}$ à support compact dans $\left(\bar{\Omega}_{T}\right)$ [resp. $\bar{\omega}_{T}$ ], et nulles au voisinage de l'arête $x=y=0$ [resp. $t=0]$. Le lemme suivant est classique.

LEMME 5.1.1. Les espaces $\dot{C}^{\infty}\left(\Omega_{T}\right)$ et $\dot{C}^{\infty}\left(\omega_{T}\right)$ sont denses dans $H_{\lambda}^{k}\left(\Omega_{T}\right)$ et $H_{\lambda}^{k}\left(\omega_{T}\right)$.

Comme A. Majda [8], nous aurons besoin du lemme d'extension suivant.

Lemme 5.1.2. Pour tout entier $N$, il existe une constante $C$ telle que: pour tout $T>0$ il existe des opérateurs de prolongement $E_{T}$ et $E_{T}^{\prime}$, qui, pour $0 \leqslant k \leqslant N$ et $\lambda \in \mathbf{R}$, envoient respectivement $H_{\lambda}^{k}\left(\Omega_{T}\right)$ dans $H_{\lambda}^{k}(\Omega)$ et $H_{\lambda}^{k}\left(\omega_{T}\right)$ dans $H_{\lambda}^{k}(\omega)$, avec une norme majorée par $C$. En outre, $E_{T} u$ [resp. $\left.E_{T}^{\prime} f\right]$ est à support dans $\bar{\Omega}_{2 T^{\prime}}[$ resp. $\left.\bar{\omega}_{2 T}\right]$.

Preuve. Soit $\chi \in C^{\infty}(\mathbf{R})$, nulle pour $t \geqslant 2 / 3$, valant 1 pour $t \leqslant 1 / 2$. Pour $u \in H_{\lambda}^{k}\left(\Omega_{T}\right)$ posons

$$
v=\chi\left(\frac{x+y}{T}\right) u, \quad w=\left(1-\chi\left(\frac{x+y}{T}\right)\right) u .
$$

On a $v \in H_{\lambda}^{k}\left(\Omega_{T}\right)$, à support dans $\bar{\Omega}_{2 T / 3}$. En outre

$$
\|v\|_{k, \lambda, T} \leqslant C\|u\|_{k, \lambda, T}
$$

avec $C$ indépendant de $k \leqslant N, \lambda \in \mathbf{R}$ et $T>0$. On prolonge $v$ par 0 , et il suffit clairement de prolonger $w$. Posons

$$
\tilde{w}(x, y, z)=T^{3 / 2} w(T x, T y, T z) .
$$

Sur le support de $w$ on a $x+y \in[T / 2, T]$; on en déduit que $\tilde{w} \in H^{k}\left(\Omega_{1}\right)$, est supporté dans $x+y \geqslant 1 / 2$, et que l'on a

$$
C^{-1}\|w\|_{k, \lambda, T} \leqslant T^{-\lambda}\|\tilde{w}\|_{k, \lambda} \leqslant C\|w\|_{k, \lambda, T}
$$

avec

$$
\|\tilde{w}\|_{k, \lambda}=\left(\sum_{|\alpha| \leqslant k}(1+|\lambda|)^{2(k-|\alpha|)}\left\|\partial^{\alpha} \tilde{w}\right\|_{I^{2}\left(\Omega_{1}\right)}^{2}\right)^{1 / 2}
$$

et $C$ indépendant de $k \leqslant N, \lambda$ et $T>0$.

Notons $E$ un opérateur de prolongement de $H^{k}\left(\Omega_{1}\right)$ dans $H^{k}(\Omega)$, pour $k=$ $0, \ldots, N$, tel que $E$ u soit supporté dans $\bar{\Omega}_{2}$. Alors $E$ est aussi borné, uniformément par rapport à $\lambda$, si on munit les espaces $H^{\lambda}$ des normes (5.1.6) \|\|$_{k, \lambda}$. Il suffit maintenant de poser

$$
E_{T} u=v+T^{-3}(E \tilde{w})\left(\frac{x}{T}, \frac{y}{T}, \frac{z}{T}\right) .
$$

La construction de $E_{T}^{\prime}$ est tout à fait similaire.

5.2. Coordonnées cylindriques. Il est naturel de passer en coordonnées cylindriques: nous posons $t=x+y, \theta=x /(x+y)$, et pour u définie sur $\Omega$

$$
J_{\lambda} u(t, \theta, z)=t^{-\lambda} u(t \theta, t(1-\theta), z) .
$$


$J_{\lambda}$ est une isométrie de $L_{\lambda+1 / 2}^{2}\left(\Omega_{T}\right)$ sur $L^{2}\left(\hat{\Omega}_{T}\right)$ si l'on note $\left.\hat{\Omega}_{T}=\right] 0, T[\times] 0,1[\times \mathbf{R}$. Plus généralement notons $\hat{H}^{k}\left(\hat{\Omega}_{T}\right)$ l'espace des $v \in L^{2}\left(\hat{\Omega}_{T}\right)$ tels que $t^{j+l} \partial_{t}^{j} \partial_{z}^{l} \partial_{\theta}^{m} v \in$ $L^{2}\left(\hat{\Omega}_{T}\right)$ pour $j+l+m \leqslant k$, que nous munissons des normes

$$
\|v\|_{k, \lambda, T}^{\prime \prime}=\left\{\sum_{j+l+m \leqslant k}(1+|\lambda|)^{2(k-j-l-m)}\left\|t^{j+l} \partial_{t}^{j} \partial_{z}^{l} \partial_{\theta}^{m} v\right\|_{L^{2}\left(\hat{\Omega}_{T}\right)}^{2}\right\}^{1 / 2}
$$

On a alors

LEMME 5.2.1. $J_{\lambda}$ est un isomorphisme de $H_{\lambda+1 / 2}^{k}\left(\Omega_{T}\right)$ sur $\hat{H}^{k}\left(\hat{\Omega}_{T}\right)$; en outre on a

$$
C^{-1}\|u\|_{k, \lambda+1 / 2, T} \leqslant\left\|J_{\lambda} u\right\|_{k, \lambda, T}^{\prime \prime} \leqslant\|u\|_{k, \lambda+1 / 2, T}
$$

avec $C$ indépendant de $\lambda$ et $T$.

Introduisons maintenant une fonction $\chi \in C_{0}^{\infty}(] \frac{1}{2}, 2[)$ telle que

$$
\sum_{j \in \mathbf{Z}} \chi\left(2^{j} t\right)=1 \text { pour } t>0
$$

A une fonction $v$ définie sur $\hat{\Omega}_{T}$ on associe les fonctions suivantes

$$
v_{j}(t, \theta, z)=\chi\left(2^{j} t\right) v(t, \theta, z) \text { pour } 2^{j+1} T>1
$$

Le lemme suivant est immédiat

LEMME 5.2.2. (i) Si $v \in \hat{H}^{k}\left(\hat{\Omega}_{T}\right)$ alors $v_{j} \in \hat{H}^{k}\left(\hat{\Omega}_{T}\right)$ et

$$
\sum_{2^{j+1} T>1}\left\|v_{j}\right\|_{k, \lambda, T}^{\prime \prime 2} \leqslant\|v\|_{k, \lambda, T}^{\prime \prime 2}
$$

(ii) Si les $w_{j} \in \hat{H}^{k}\left(\hat{\Omega}_{T}\right)$ sont à support dans $\gamma^{1} 2^{-j} \leqslant t \leqslant \gamma 2^{j}$ et vérifient $\sum\left\|w_{j}\right\|_{k, \lambda, T}^{\prime \prime 2}$ $<+\infty$, alors $v=\sum_{j} w_{j} \in \hat{H}^{k}\left(\hat{\Omega}_{T}\right)$ et

$$
\|v\|_{k, \lambda, T}^{2} \leqslant C \sum_{j}\left\|w_{j}\right\|_{k, \lambda, T}^{2}
$$

Dans (5.2.5) (5.2.6) les constantes $C$ sont indépendantes de $T>0$ et $\lambda \in \mathbf{R}$.

On complète maintenant l'opération de découpage (5.2.4) par des dilatations: on pose

$$
\tilde{v}_{j}(t, \theta, z)=2^{-j} v_{j}\left(2^{-j} t, \theta, 2^{-j} z\right) .
$$

Notons $T_{j}=\operatorname{Inf}\left(2, T 2^{j}\right), \tilde{\Omega}_{T_{j}}$ l'ouvert $\left[-\infty, T_{j}[\times] 0,1\left[\times \mathbf{R}\right.\right.$ et $\|\cdot\|_{k, \lambda}^{\prime}$ la norme sur $H^{k}\left(\tilde{\Omega}_{T_{j}}\right)$ définie par

$$
\|w\|_{k, \lambda}^{\prime}=\left(\sum_{|\alpha| \leqslant k}(1+|\lambda|)^{2(k-|\alpha|)}\left\|\partial^{\alpha} w\right\|_{L^{2}\left(\tilde{\Omega}_{T_{j}}\right)}^{2}\right)^{1 / 2} .
$$

On remarquera que $T_{j}>\frac{1}{2}$ pour $2^{j+1} T>1$, et que $\tilde{v}_{j}$ est à support dans ]- $\frac{1}{2}, T_{j}$ ]. On en déduit aussitôt

LEMME 5.2.3. (i) Si $v \in \hat{H}^{k}\left(\Omega_{T}\right)$ alors $\tilde{v}_{j} \in H^{k}\left(\tilde{\Omega}_{T_{j}}\right)$ et

$$
\sum_{2^{j+1} T>1}\left\|\tilde{v}_{j}\right\|_{k, \lambda}^{2} \leqslant C\|v\|_{k, \lambda, T}^{\prime \prime}
$$


(ii) Si les $\tilde{w}_{j} \in H^{k}\left(\tilde{\Omega}_{T_{j}}\right)$, pour $T 2^{j+1}>1$, sont à support dans $t \geqslant \gamma>0$, et vérifient $\Sigma\left\|w_{j}\right\|_{k, \lambda}^{\prime \prime 2}<+\infty$, alors

$$
v(t, \theta, z)=\sum_{j} 2^{j} w_{j}\left(2^{j} t, \theta, 2^{j} z\right) \in \hat{H}^{k}\left(\Omega_{T}\right)
$$

avec

$$
\|v\|_{k, \lambda, T}^{\prime \prime} \leqslant C\left\{\sum_{j}\left\|w_{j}\right\|_{k, \lambda}^{\prime \prime 2}\right\}^{2} .
$$

Dans (5.2.9), (5.2.10) les constantes $C$ sont indépendantes de $\lambda$ et $T>0$.

On peut répéter les opérations ci-dessus pour les fonctions $\phi$ définies sur $\omega$ : on définit

$$
J_{\lambda} \phi(t, z)=t^{-\lambda} \phi(t, z) .
$$

Notant $\hat{H}^{k}\left(\omega_{T}\right)$ l'espace des fonctions $\phi$ telles que $t^{j+l} \partial_{t}^{j} \partial_{z}^{\prime} \phi \in L^{2}\left(\omega_{T}\right)$ pour $j+l \leqslant k$, muni des normes

$$
\|\phi\|_{k, \lambda, T}^{\prime \prime}=\left\{\sum_{j+l \leqslant k}(1+|\lambda|)^{2(k-j-l)}\left\|t^{j+l} \partial_{t}^{j} \partial_{z}^{l} f\right\|_{L^{2}\left(\omega_{T}\right)}^{2}\right\}^{1 / 2} .
$$

On a

LEMME 5.2.4. $J_{\lambda}$ est un isomorphisme de $H_{\lambda}^{k}\left(\omega_{T}\right) \operatorname{sur} \hat{H}^{k}\left(\omega_{T}\right)$ et

$$
C^{-1}\|\phi\|_{k, \lambda, T} \leqslant\left\|J_{\lambda} \phi\right\|_{k, \lambda, T}^{\prime \prime} \leqslant C\|\phi\|_{k, \lambda, T}
$$

avec $C$ indépendant de $\lambda$ et $T$.

Pour $\Psi \in \hat{H}^{k}\left(\omega_{T}\right)$ on définit

$$
\begin{gathered}
\Psi_{j}(t, z)=\chi\left(2^{j} t\right) \phi(t, z) \quad \text { pour } T 2^{j+1}>1 \\
\tilde{\Psi}_{j}(t, z)=2^{-j} \phi_{j}\left(2^{-j} t, 2^{-j} z\right) .
\end{gathered}
$$

Notons maintenant $\left.\tilde{\omega}_{T_{j}}=\right]-\infty, T_{j}\left[\times \mathbf{R}\right.$ et munissons $H^{k}\left(\tilde{\omega}_{T_{j}}\right)$ des normes

$$
\|f\|_{k, \lambda}^{\prime}=\left\{\sum_{m+l \leqslant k}(1+|\lambda|)^{2(k-m-l)}\left\|\partial_{t}^{m} \partial_{z}^{l} f\right\|_{L^{2}\left(\tilde{\omega}_{T_{j}}\right)}^{2}\right\}^{1 / 2} .
$$

Les Lemmes 5.2.2 et 5.2.3 sont encore vrais mot pour mot, si l'on change $\hat{\Omega}_{T}$ par $\omega_{T}$ et $\tilde{\Omega}_{T_{i}}$ par $\tilde{\omega}_{T_{j}}$.

5.3. Multiplication par une fonction de $H^{\prime}$.

Proposition 5.3.1. Soit $l$ un entier $\geqslant 2$. Il existe $C$ el que pour $k \leqslant l, T \leqslant T_{0}$, $\lambda \in \mathbf{R}, a \in H^{\prime}\left(\Omega_{T_{0}}\right)\left[\right.$ resp. $\left.b \in H^{l}\left(\omega_{T_{0}}\right)\right]$ et $u \in H_{\lambda}^{k}\left(\Omega_{T}\right)\left[\right.$ resp. $\left.\phi \in H_{\lambda}^{k}\left(\omega_{T}\right)\right]$, on $a$ $u \in H^{k}\left(\Omega_{T}\right)\left[\right.$ resp. $\left.b \phi \in H_{\lambda}^{k}\left(\omega_{T}\right)\right]$ avec

$$
\|a u\|_{k, \lambda, T} \leqslant\|a\|_{H^{\prime}\left(\Omega_{T_{0}}\right)}\|u\|_{k, \lambda, T}
$$

$\left[\right.$ resp. $\left.\|b \phi\|_{k, \lambda, T} \leqslant C\|b\|_{H^{\prime}\left(\omega_{T_{0}}\right)}\|\phi\|_{k, \lambda, T}\right]$. 
Commençons par établir un lemme de plongement

LEMME 5.3.2 Etant donnés $l$ et $T_{0}>0$, il existe $C$ tel que: pour $k \leqslant l, \lambda \in \mathbf{R}$, $T \leqslant T_{0}, u \in H_{\lambda}^{k}\left(\Omega_{T}\right)$ et $q>2$ vérifiant $\frac{1}{2} \geqslant \frac{1}{q} \geqslant \operatorname{Max}\left(0, \frac{1}{2}-\frac{k}{3}\right)$, on $a$

$$
(1+|\lambda|)^{k-3 \rho}\left\|(x+y)^{-\lambda+3 \rho} u\right\|_{L^{q}\left(\Omega_{T}\right)} \leqslant C\|u\|_{k, \lambda, T}
$$

avec $\rho=\frac{1}{2}-\frac{1}{4}$.

Preuve. On passe en coordonnées polaires: on pose $v=J_{\lambda-1 / 2} u$ et on définit $v_{j}$ et $\tilde{v}_{j}$ par les formules (5.2.4) (5.2.7). Posant $\varepsilon_{\mathrm{j}}=\left\|\tilde{v}_{j}\right\|_{k, \lambda}^{\prime}$, on a donc

$$
\left\|\left(\varepsilon_{j}\right)\right\|_{l^{2}} \leqslant C\|u\|_{k, \lambda, T}
$$

Les conditions de support des $v_{j}$ montrent que

$$
\left\|(x+y)^{-\lambda+3 \rho} u\right\|_{L^{q}\left(\Omega_{T}\right)}^{q}=\left\|t^{2 \rho} v\right\|_{L^{q}\left(\Omega_{T}\right)}^{q} \leqslant C \sum_{j}\left\|v_{j}\right\|_{L^{q}\left(\Omega_{T_{j}}\right)}^{q} .
$$

Pour $w \in H^{k}\left(\tilde{\Omega}_{T_{j}}\right)$ on a les injections de Sobolev et les inégalités suivantes:

$$
(1+|\lambda|)^{k-3 \rho}\|w\|_{L^{q}} \leqslant C\left\{\|w\|_{H^{k}}+(1+|\lambda|)^{k}\|w\|_{L^{2}}\right\}
$$

pour $\rho=\frac{1}{2}-\frac{1}{q}$ et $\frac{1}{2} \geqslant \frac{1}{q} \geqslant \operatorname{Max}\left(0, \frac{1}{2}-\frac{\dot{\alpha}}{3}\right)$.

On a donc

$$
(1+|\lambda|)^{k-3 \rho}\left\|\tilde{v}_{j}\right\|_{L^{4}} \leqslant C\left\|\tilde{v}_{j}\right\|_{k, \lambda}^{\prime}
$$

et avec (5.3.2) (5.3.1) et l'inégalité $\left\|\left(\varepsilon_{j}\right)\right\|_{l^{4}} \leqslant\left\|\left(\varepsilon_{j}\right)\right\|_{l^{2}}$, on en déduit le lemme.

Lemme 5.3.3. Etant donnés $l$ et $T_{0}>0$ il existe $C$ tel que: pour $k \leqslant l, \lambda \in \mathbf{R}$, $T \leqslant T_{0}, \phi \in H_{\lambda}^{k}\left(\omega_{T}\right)$ et $q \geqslant 2$ vérifiant

$$
\begin{aligned}
& \frac{1}{2} \geqslant \frac{1}{q} \geqslant 0 \quad \text { si } k \geqslant 2, \\
& \frac{1}{2} \geqslant \frac{1}{q}>0 \quad \text { si } k=1 .
\end{aligned}
$$

On $a$

$$
(1+|\lambda|)^{k-2 \rho}\left\|t^{-\lambda+2 \rho} \phi\right\|_{L^{q}\left(\omega_{T}\right)} \leqslant C\|\phi\|_{k, \lambda, T}
$$

Preuve. Elle est similaire à celle du lemme précédent, en posant $\Psi=J_{\lambda} \phi$, et définissant $\Psi_{j}$ et $\tilde{\Psi}_{j}$ par (5.2.13) et (5.2.14). Il faut seulement, lorsque $k=1$, faire attention au cas limite de l'injection de Sobolev de $H^{k}\left(\tilde{\omega}_{T_{j}}\right)$ dans $L^{q}\left(\tilde{\omega}_{T_{j}}\right)$.

LEMME 5.3.4. Etant donnés l et $T_{0}>0$, il existe $C$ tel que: pour $k \leqslant l, m \leqslant l$, avec $k+m \geqslant 2$, pour $\lambda \in \mathbf{R}, T \leqslant T_{0}, a \in H^{m}\left(\Omega_{T_{0}}\right)$ et $u \in H_{\lambda}^{k}\left(\Omega_{T}\right)$ on $a$

(i) Si $m \geqslant 2$, a $u \in L_{\lambda}^{2}\left(\Omega_{T}\right)$ avec

$$
(1+|\lambda|)^{k}\|a u\|_{0, \lambda, T} \leqslant C\|a\|_{H^{m}\left(\Omega_{T_{0}}\right)}\|u\|_{k, \lambda, T}
$$

(ii) Si $m \leqslant 1$ alors a $u \in L_{\lambda-3 / 2+m}^{2}\left(\Omega_{T}\right)$ avec

$$
(1+|\lambda|)^{k-3 / 2+m}\|a u\|_{0, \lambda-3 / 2+m, T} \leqslant C\|a\|_{H^{m}\left(\Omega_{T_{0}}\right)}\|u\|_{k, \lambda, T}
$$


PreUve. Si $m \geqslant 2$ alors $a \in L^{\infty}\left(\Omega_{T_{0}}\right)$ et (5.3.5) est immédiat. Si $m \leqslant 1$ alors $a \in L^{p}\left(\Omega_{T_{0}}\right)$ pour $\frac{1}{p}=\frac{1}{2}-\frac{m}{3}>0$. Puisque $k+m \geqslant 2$, on peut appliquer le Lemme 5.3.2 avec $\frac{1}{4}=\frac{1}{2}-\frac{1}{p}=\frac{m}{3}>\frac{1}{2}-\frac{k}{3}$, et on obtient (5.3.6) par l'inégalité de Hölder.

On a un énoncé analogue pour $b \in H^{m}\left(\omega_{T_{0}}\right)$ et $\phi \in H_{\lambda}^{k}\left(\omega_{T}\right)$ avec $m+k \geqslant 2$. Il faut cependant remplacer (5.3.6) par

$$
(1+|\lambda|)^{k-\rho}\|b \phi\|_{0, \lambda-\rho, T} \leqslant C\|b\|_{H^{m}\left(\omega_{T_{0}}\right)}\|\phi\|_{k, \lambda, T}
$$

avec $\rho=1$ si $m=0$ et $\rho$ arbitraire dans $] 0,1]$ si $m=1$, à cause du cas limite de l'injection de $H^{m}\left(\omega_{T_{0}}\right)$ dans $L^{p}\left(\omega_{T_{0}}\right)$.

Démonstration de la Proposition 5.3.1. Soit $\alpha$ une dérivation de longueur $|\alpha| \leqslant k$. Il suffit d'obtenir une estimation du type

$$
(1+|\lambda|)^{k-|\alpha|}\left\|\partial^{\beta} a \partial^{\alpha-\beta} u\right\|_{0, \lambda-|\alpha|, T} \leqslant C\|a\|_{H^{\prime}\left(\Omega_{T_{0}}\right.}\|u\|_{k, \lambda, T}
$$

pour $\beta \leqslant \alpha$. On a $\partial^{\beta} a \in H^{l-|\beta|}\left(\Omega_{T_{0}}\right)$ et $v=\partial^{\alpha-\beta} u \in H_{\lambda-|\alpha|+|\beta|}^{k-|\alpha|+|\beta|}\left(\Omega_{T}\right)$ avec

$$
\begin{gathered}
\left\|\partial^{\beta} a\right\|_{H^{\prime-|\beta|}\left(\Omega_{T_{0}}\right)} \leqslant\|a\|_{H^{\prime}\left(\Omega_{T_{0}}\right)} \\
\|v\|_{k-|\alpha|+|\beta|, \lambda-|\alpha|+|\beta|, T} \leqslant\|u\|_{k, \lambda, T}
\end{gathered}
$$

Le Lemme 5.3.4 permet de majorer par le membre de droite de (5.3.8), la quantité

$$
(1+|\lambda|)^{k-|\alpha|+|\beta|-\rho}\left\|\left(\partial^{\beta} a\right) \cdot v\right\|_{0, \lambda-|\alpha|+|\beta|-\rho, T}
$$

avec $\rho=0$ si $l-|\beta| \geqslant 2$ et $\rho=\frac{3}{2}-l+|\beta|$ si $l-|\beta| \leqslant 1$. Puisque $|\beta|-\rho \geqslant 0$, la majoration (5.3.8) en découle, si l'on fait la remarque triviale suivante

$$
\|w\|_{0, \mu, T} \leqslant C\|w\|_{0, \mu+\sigma, T}
$$

avec $C$ indépendant de $T \leqslant T_{0}$, et de $\mu \in \mathbf{R}$, pourvu que $0 \leqslant \sigma \leqslant l$.

Pour $b \in H^{I}\left(\omega_{T_{0}}\right)$ et $\phi \in H_{\lambda}^{k}\left(\omega_{T}\right)$, la démonstration est parfaitement similaire.

Nous terminons ce paragraphe par une extension de la Proposition 5.3.1

Proposition 5.3.5. Soit $g_{0}(\theta) \in C^{\infty}([0,1])$ et soit $g(\theta, X)$, définie sur $[0,1] \times \Omega_{T_{0}}$ telle que $\theta \rightarrow g(\theta, \cdot)$ soit $C^{\infty}$ sur $[0,1]$ à valeurs dans $H^{\prime}\left(\Omega_{T_{0}}\right)$. On pose alors $a(x, y, z)=g(x /(x+y), x, y, z)+g_{0}(x /(x+y))$.

Si $l \geqslant 2$, il existe $C$ tel que: pour tous $k \leqslant l, T \leqslant T_{0}, \lambda \in \mathbf{R}$ et $u \in H_{\lambda}^{k}\left(\Omega_{T}\right)$ on a $u \in H_{\lambda}^{k}\left(\Omega_{T}\right)$ avec

$$
\|a u\|_{k, \lambda, T} \leqslant C\|u\|_{k, \lambda, T} .
$$

PReuve. Par décomposition sur les polynômes de Legendre on peut écrire

$$
g(\theta, X)=\sum_{j=1}^{\infty} g_{j}(\theta) a_{j}(X)
$$

avec $\sum\left\|a_{j}\right\|_{H^{\prime}\left(\Omega_{T_{0}}\right)}<+\infty$, la suite $g_{j}$ étant bornée dans $C^{\infty}([0,1])$.

Avec la Proposition 5.3.1, on voit qu'il nous reste à établir la Proposition 5.3.5 pour $a(x, y, z)=g(x /(x+y))$ avec $g \in C^{\infty}([0,1])$. Dans ce cas on a

$$
\left|\partial^{\alpha} a(x, y, z)\right| \leqslant C|x+y|^{-|\alpha|}
$$

et l'estimation (5.3.12) s'en déduit directement à partir de la formule de Leibniz. 


\subsection{Propriétés d'algèbre.}

Proposition 5.4.1. Soit $k$ entier, $k \geqslant 2$. Il existe $C$ tel que pour tous $\lambda, u \in$ $H_{\lambda}^{k}\left(\Omega_{T}\right), u^{\prime} \in H_{\lambda}^{k}\left(\Omega_{T}\right)$ [resp. $\phi \in H_{\lambda}^{k}\left(\omega_{T}\right), \phi^{\prime} \in H_{\lambda}^{k}\left(\omega_{T}\right)$ ] le produit uu' est dans $H_{2 \lambda-3 / 2}^{k}\left(\Omega_{T}\right)\left[\right.$ resp. $\left.\phi \phi^{\prime} \in H_{2 \lambda-1}^{k}\left(\omega_{T}\right)\right]$ et

$$
\left\|u u^{\prime}\right\|_{k, 2 \lambda-3 / 2, T} \leqslant C\|u\|_{k, \lambda, T}\left\|u^{\prime}\right\|_{k, \lambda, T}
$$

[resp. $\left.\left\|\phi \phi^{\prime}\right\|_{k, 2 \lambda-1, T} \leqslant C\|\phi\|_{k, \lambda, T}\left\|\phi^{\prime}\right\|_{k, \lambda, T}\right]$.

Preuve. A nouveau, on n'écrit la preuve que pour les fonctions de $H_{\lambda}^{k}\left(\Omega_{T}\right)$. On utilise les coordonnées polaires et on pose $v=J_{\lambda-1 / 2} u, v^{\prime}=J_{\lambda-1 / 2} u^{\prime}$. On applique ensuite aux fonctions $v$ et $v^{\prime}$ la décomposition (5.2.4). Introduisons la fonction $w=J_{2 \lambda-2}\left(u u^{\prime}\right)=t v v^{\prime}=\sum w_{j}$ avec

$$
w_{j}=v_{j} \sum_{\left|j^{\prime}-j\right| \leqslant 1} t v_{j^{\prime}}^{\prime}
$$

On applique aux fonctions $w_{j}$ et $v_{j}$ les dilations (5.2.7) et on note $\tilde{w}_{j}=\tilde{v}_{j} \cdot \tilde{v}_{j}^{\prime}$ avec

$$
\tilde{v}_{j}^{\prime}(t, \theta, z)=\sum_{\left|j^{\prime}-j\right| \leqslant 1} 2^{-j} t v_{j^{\prime}}^{\prime}\left(2^{-j} t, \theta, 2^{-j} z\right) .
$$

Comme au Lemme 5.2.3 on vérifie que

$$
\sum_{j}\left\|\tilde{v}_{j}^{\prime}\right\|_{k, \lambda}^{\prime 2} \leqslant C\left\|v^{\prime}\right\|_{k, \lambda, T}^{\prime 2}
$$

Puisque $k \geqslant 2, H^{k}\left(\tilde{\Omega}_{T_{j}}\right)$ est une algèbre et

$$
\left\|\tilde{w}_{j}\right\|_{k, \lambda}^{\prime} \leqslant C\left\|\tilde{v}_{j}\right\|_{k, \lambda}^{\prime}\left\|\tilde{v}_{j}^{\prime}\right\|_{k, \lambda}^{\prime} .
$$

Avec le Lemme 5.2.3 et (5.4.3) on en tire que

$$
\sum_{j}\left\|\tilde{w}_{j}\right\|_{k, \lambda}^{\prime 2} \leqslant C\|v\|_{k, \lambda, T}^{\prime 2}\left\|v^{\prime}\right\|_{k, \lambda, T}^{\prime 2}
$$

Il résulte alors des Lemmes 5.2.1 et 5.2.3 que $u \in H_{2 \lambda-3 / 2}^{k}\left(\Omega_{T}\right)$ avec l'estimation annoncée.

Proposition 5.4.2. Soit $f$ une fonction $C^{\infty}$ sur $\mathbf{R}$ telle que $f(0)=0$. On se donne $T_{0}>0, k \geqslant 2$ et $K>0$. Alors il existe $C$ tel que pour tout $\lambda \geqslant k$, tout $u \in H_{\lambda}^{k}\left(\Omega_{T_{0}}\right)$ $\left[\right.$ resp. $\left.\phi \in H_{\lambda}^{k}\left(\omega_{T_{0}}\right)\right]$, à valeurs réelles, et vérifiant

$$
\|u\|_{k, \lambda, T_{0}} \leqslant K \quad\left[\text { resp. }\|\phi\|_{k, \lambda, T_{0}} \leqslant K\right] .
$$

On $a$

(i) $f(u) \in H_{\lambda}^{k}\left(\Omega_{T_{0}}\right)\left[\right.$ resp. $\left.f(\phi) \in H_{\lambda}^{k}\left(\omega_{T_{0}}\right)\right]$, et pour $T \leqslant T_{0}$,

$$
\|f(u)\|_{k, \lambda, T} \leqslant C\|u\|_{k, \lambda, T} \quad\left[\text { resp. }\|f(\phi)\|_{k, \lambda, T} \leqslant C\|\phi\|_{k, \lambda, T}\right]
$$

(ii) $v=f(u)-u f^{\prime}(u) \in H_{2 \lambda-3 / 2}^{k}\left(\Omega_{T_{0}}\right)\left[\right.$ resp. $\left.\Psi=f(\phi)-\phi f^{\prime}(\phi) \in H_{2 \lambda-1}^{k}\left(\omega_{T_{0}}\right)\right]$ et pour $T \leqslant T_{0}$,

$$
\|v\|_{k, 2 \lambda-3 / 2, T} \leqslant C\|u\|_{k, \lambda, T}^{2},
$$

$\left[\right.$ resp. $\left.\|\Psi\|_{k, 2 \lambda-1, T} \leqslant C\|\phi\|_{k, \lambda, T}^{2}\right]$. 
Preuve. Puisque $\lambda \geqslant k$, on a

$$
\|u\|_{H^{k}\left(\Omega_{T_{0}}\right)} \leqslant C\|u\|_{k, \lambda, T_{0}} \leqslant C \cdot K .
$$

Ecrivant $f(\xi)=\xi \cdot g(\xi)$, on a $\|g(u)\|_{L^{\infty}\left(\Omega_{T_{0}}\right)} \leqslant C_{1}$ et on en déduit que

$$
(1+|\lambda|)^{k}\|f(u)\|_{L_{\lambda}^{2}\left(\Omega_{T}\right)} \leqslant C_{1}\|u\|_{k, \lambda, T}
$$

Pour $0<|\alpha| \leqslant k$ on écrit $\partial^{\alpha} f(u)$ comme une somme de termes

$$
w=f^{(q)}(u) \partial^{\alpha_{1}} u \cdots \partial^{\alpha_{q}} u
$$

avec $\left|\alpha_{1}\right|+\cdots+\left|\alpha_{q}\right|=|\alpha|$ et $\left|\alpha_{j}\right|>0$. Le terme $f^{(q)}(u)$ est borné dans $L^{\infty}\left(\Omega_{T_{0}}\right)$. Si $q=1$, alors $\left|\alpha_{1}\right|=|\alpha|$ et on estime le terme (5.4.8) par

$$
(1+|\lambda|)^{k-|\alpha|}\|w\| L_{k-|\alpha|}^{2}\left(\Omega_{T}\right) \leqslant C\|u\|_{k, \lambda, T}
$$

Si $q=2$ on écrit que $\partial^{\alpha_{1}} u \in H^{k-\left|\alpha_{1}\right|}\left(\Omega_{T_{0}}\right)$ et que $\partial^{\alpha_{2}} u \in H_{\lambda-\left|\alpha_{2}\right|}^{k-\left|\alpha_{2}\right|}$. Le Lemme 5.3.4 et l'inégalité (5.3.11) permettent d'obtenir à nouveau l'estimation (5.4.9).

Si $q \geqslant 3$ alors $k-\left|\alpha_{j}\right| \geqslant 2$ pour $j=1, \ldots, q$. On écrit que $\partial^{\alpha_{1}} u, \ldots, \partial_{q-1}^{\alpha} u$ sont bornés dans $L^{\infty}\left(\Omega_{T_{0}}\right)$, alors que $\partial^{\alpha_{q}} u \in H_{\lambda-\left|\alpha_{q}\right|}^{k-\left|\alpha_{q}\right|}$. On en tire encore la majoration (5.4.9).

Finalement (5.4.9) et (5.4.7) donnent le point (i) de la proposition.

Pour obtenir le point (ii) il suffit d'écrire que $f(\xi)-\xi f^{\prime}(\xi)=\xi \cdot g(\xi)$ où $g \in$ $C^{\infty}(\mathbf{R})$ avec $g(0)=0$. On applique le point (i) à $g(u)$, et on conclut grâce à la Proposition 5.4.1.

La Proposition 5.4.2 se généralise aussitôt au cas où $f$ est $C^{\infty}$ sur $\mathbf{R}^{n}$ et les fonctions $u$ et $\phi$ sont à valeurs dans $\mathbf{R}^{n}$; on peut également supposer que $f$ dépend de façon $C^{\infty} \operatorname{de}(x, y, z) \in \bar{\Omega}_{T_{0}}$ ou de $(t, z) \in \bar{\omega}_{T_{0}}$.

\section{Le problème de Goursat linéaire.}

6.1. Notations et résultats. Nous reprenons les notations du paragraphe 3.3 et nous étudions un système (3.3.3) linéarisé, du type suivant

$$
\left\{\begin{array}{c}
L u=A \frac{\partial u}{\partial x}+B \frac{\partial u}{\partial y}+C \frac{\partial u}{\partial z} \\
F^{\prime}\left(\gamma^{\prime} u, \phi^{\prime}\right)=l^{\prime} \frac{\partial \phi^{\prime}}{\partial t}+p^{\prime} \frac{\partial \phi^{\prime}}{\partial z}+q^{\prime} \phi^{\prime}+m^{\prime} \gamma^{\prime} u \\
F^{\prime \prime}\left(\gamma^{\prime \prime} u, \phi^{\prime \prime}\right)=l^{\prime \prime} \frac{\partial \phi^{\prime \prime}}{\partial t}+p^{\prime \prime} \frac{\partial \phi^{\prime \prime}}{\partial t}+p^{\prime \prime} \frac{\partial \phi^{\prime \prime}}{\partial z}+q^{\prime \prime} \phi^{\prime \prime}+m^{\prime \prime} \gamma^{\prime \prime} u
\end{array}\right.
$$

où les matrices $2 \times 2, A, B$ et $C$ sont réelles, de classe $C^{2}$ sur $\Omega_{T_{0}}$, et où les vecteurs $l^{\prime}, p^{\prime}, q^{\prime}, l^{\prime \prime}, p^{\prime \prime}, q^{\prime \prime}$ et les matrices $m^{\prime}$ et $m^{\prime \prime}$ sont réelles et de classe $C^{2}$ sur $\omega_{T_{0}}$.

Par ailleurs, on se donne un système $(\underline{L}, \underline{F})$ comme (6.1.1) à coefficients $\underline{A}$, $\underline{B}, \ldots, \underline{l}^{\prime}, \ldots, \underline{m}^{\prime \prime}$ constants. Nous supposerons tout au long de cette partie que le système $(\underline{L}, \underline{F})$ satisfait aux hypothèses $(\alpha)$ et $(\beta)$ du paragraphe 3.3.

Notre but est de résoude le système

$$
L u=f, \quad F(\omega, \phi)=g ; \quad \phi_{\mid t=0}=0,
$$

ce que nous ferons en supposant $(L, F)$ voisin de $(\underline{L}, \underline{F})$. 
Nous noterons $\varepsilon(L, F)$ le maximum de $\|A-\underline{A}\|_{L^{\infty}\left(\Omega_{T_{0}}\right)}\|B-\underline{B}\|_{L^{\infty}\left(\Omega_{T_{0}}\right)}, \ldots$, $\|l-l\|_{L^{\infty}\left(\omega_{T_{0}}\right)}, \ldots,\|m-\underline{m}\|_{L^{\infty}\left(\omega_{T_{0}}\right)}$.

Enfin, nous nous donnons un entier $N \geqslant 4$ et nous supposons que $A-\underline{A}, B-\underline{B}$ et $C-\underline{C}$ sont dans $H^{N}\left(\Omega_{T_{0}}\right)$, et que $l-\underline{l}, \ldots, m-\underline{m}$ sont dans $H^{N}\left(\omega_{T_{0}}\right)$. On note $\|L, F\|_{N}$ le maximum des normes $H^{N}$ de ces fonctions.

Pour terminer, introduisons deux autres notations: $W_{\lambda, T}^{k}$ désigne l'espace des couples $(u, \phi) \in H_{\lambda+1 / 2}^{k}\left(\Omega_{T}\right) \times H_{\lambda+1}^{k+1}\left(\omega_{T}\right)$ tels que $\gamma u \in H_{\lambda}^{k}\left(\omega_{T}\right)$. Cet espace est muni de la norme

$$
\|u, \phi\|_{k, \lambda, T}=\left\{\lambda\|u\|_{k, \lambda+1 / 2, T}^{2}+\|\gamma u\|_{k, \lambda, T}^{2}+\|\phi\|_{k+1, \lambda+1, T}^{2}\right\}^{1 / 2} .
$$

De même $W_{\lambda, T}^{\prime k}$ désigne l'espace des couples $(f, g) \in H_{\lambda-1 / 2}^{k}\left(\Omega_{T}\right) \times H_{\lambda}^{k}\left(\omega_{T}\right)$; il est muni de la norme

$$
\|f, g\|_{k, \lambda, T}^{\prime}=\left\{\lambda^{-1}\|f\|_{k, \lambda-1 / 2, T}^{2}+\|g\|_{k, \lambda, T}^{2}\right\} .
$$

ThEORÈm 6.1.1. Sous les hypothèses ci-dessus, il existe $\varepsilon_{0}>0$ et des fonctions $\lambda_{0}(K), C_{0}(K)$ telles que si l'on a,

$$
\varepsilon(I, F) \leqslant \varepsilon_{0}, \quad\|L, F\|_{N} \leqslant K,
$$

alors, pour tour $\lambda \geqslant \lambda_{0}(K)$, tout $T \leqslant T_{0}$, tout $(f, g) \in W_{\lambda, T}^{\prime k}$ avec $k \leqslant N$, le système (6.1.2) possède une unique solution $(u, \phi) \in W_{\lambda, T}^{k}$ qui vérifie

$$
\|u, \phi\|_{k, \lambda, T} \leqslant C_{0}(K)\|f, g\|_{k, \lambda, T}^{\prime} .
$$

En outre, si f et $g$ sont réelles, il en est de même de $(u, \phi)$.

Pour démontrer ce théorème l'idée essentielle est de passer en coordonnées cylindriques, puis d'effectuer les partitions de l'unité et les dilatations comme au paragraphe 5.2, pour se ramener à un problème mixte du type de ceux étudiés par A. Majda [7]. Pour plus de clarté nous énonçons d'abord le résultat concernant ce problème mixte et nous reviendrons ensuite au Théorème 6.1.1.

6.2. Un théorème de $A$. Majda. Notons $(t, \theta, z)$ la variable de $\tilde{\Omega}=\mathbf{R} \times] 0,1[\times \mathbf{R}$ et considérons un opérateur de la forme

$$
\tilde{L}=\frac{\partial u}{\partial t}+G \frac{\partial u}{\partial \theta}+\rho(t) C \frac{\partial u}{\partial z}
$$

où $G=(1-\theta) G_{0}+\theta G_{1}$. Les matrices $G_{0}, G_{1}$ et $C$ sont supposées de classe $C^{2}$ sur $\tilde{\Omega}$, bornées ainsi que leurs dérivées; $\rho$ est une fonction strictement positive, $C^{\infty}$ et constante hors d'un compact.

On désigne par $\gamma u=\left(\gamma^{\prime} u, \gamma^{\prime \prime} u\right)$ les traces de $u$ sur les bords $\theta=0$ et $\theta=1$ et on adjoint à (6.2.1) des conditions aux limites

$$
\left\{\begin{array}{c}
\tilde{F}^{\prime}\left(\gamma^{\prime} u, \phi^{\prime}\right)=\tilde{l}^{\prime} \frac{\partial \phi^{\prime}}{\partial t}+\rho(t) \tilde{p}^{\prime} \frac{\partial \phi^{\prime}}{\partial z}+\tilde{q}^{\prime} \phi^{\prime}+\tilde{m}^{\prime} \gamma^{\prime} u, \\
\tilde{F}^{\prime \prime}\left(\gamma^{\prime \prime} u, \phi^{\prime \prime}\right)=\tilde{l}^{\prime \prime} \frac{\partial \phi^{\prime \prime}}{\partial t}+\rho(t) \tilde{p}^{\prime \prime} \frac{\partial \phi^{\prime \prime}}{\partial z}+\tilde{q}^{\prime \prime} \phi^{\prime \prime}+\tilde{m}^{\prime \prime} \gamma^{\prime \prime} u,
\end{array}\right.
$$

les vecteurs $\tilde{l}$ et $\tilde{p}$ étant de classe $C^{2}$ sur $\mathbf{R}^{2}, \tilde{q}$ et $\tilde{m}$ étant de classe $C^{1}$, bornés à dérivées bornées. 
Par ailleurs on se donne $(\underline{L}, \underline{F})$ un système du type de (6.2.1) (6.2.2) où les coefficients $G_{0}, G_{1}, \underline{C}, \underline{l}, \underline{p}, \underline{m}$ sont constants et où $q=0$.

Nous faisons les hypothèses suivantes:

( $\tilde{\alpha}) \underline{\tilde{L}}$ est strictement hyperbolique dans la direction $\partial_{t}$.

$(\tilde{\beta})$ Les problèmes suivants sont uniformément stables au sens de Majda:

$$
\begin{aligned}
& \left\{\begin{array}{l}
\frac{\partial u}{\partial t}+\underline{G}_{0} \frac{\partial u}{\partial \theta}+\underline{C} \frac{\partial u}{\partial z}=f \quad \text { dans } \theta>0 \\
\underline{\tilde{l}}^{\prime} \frac{\partial \phi^{\prime}}{\partial t}+\underline{\tilde{p}}^{\prime} \frac{\partial \phi^{\prime}}{\partial z}+\underline{\tilde{m}}^{\prime} u_{\mid \theta=0}=g^{\prime}
\end{array}\right. \\
& \left\{\begin{array}{l}
\frac{\partial u}{\partial t}+G_{1} \frac{\partial u}{\partial \theta}+\underline{C} \frac{\partial u}{\partial z}=f \quad \text { dans } \theta<1, \\
\tilde{l}^{\prime \prime} \frac{\partial \phi^{\prime \prime}}{\partial t}+\tilde{p}^{\prime \prime} \frac{\partial \phi^{\prime \prime}}{\partial z}+\underline{\tilde{m}}_{\mid \theta=1}^{\prime \prime}=g^{\prime \prime}
\end{array}\right.
\end{aligned}
$$

Nous notons $\varepsilon(\tilde{L}, \tilde{F})$ un majorant de $\left\|G_{0}-G_{0}\right\|_{L^{\infty}(\tilde{\Omega})},\left\|G_{1}-\bar{G}_{1}\right\|_{L^{\infty}(\tilde{\Omega})}$, $\|C-\underline{C}\|_{L^{\infty}(\tilde{\Omega})}, \quad\|l-\underline{l}\|_{L^{\infty}\left(R^{2}\right)}, \quad\|p-\underline{p}\|_{L^{\infty}\left(\mathbf{R}^{2}\right)}$ et $\|m-\underline{m}\|_{L^{\infty}\left(\mathbf{R}^{2}\right)}$ (on notera que $\varepsilon(\tilde{L}, \tilde{F})$ ne dépend pas de la fonction $\rho(t))$, enfin désignons par $\|\tilde{L}, \tilde{F}\|_{C^{2}}$ un majorant des normes $C^{2}$ de $G_{0}, G_{1}, C, l, p$ et des normes $C^{1}$ de $R, q, m$.

$\lambda$ étant un paramètre assez grand, nous étudions le problème suivant

$$
\tilde{L} u+\lambda u=f, \quad \tilde{F}(\gamma u, \phi)+(\lambda+1) \phi=g .
$$

ThEORÈmE 6.2.1. Sous les hypothèses $(\tilde{\alpha}),(\tilde{\beta})$ ci-dessus, il existe $\varepsilon_{0}>0$ et des fonctions $\lambda_{0}(K)$ et $C_{0}(K)$ telles que si l'on, a

$$
\varepsilon(\tilde{L}, \tilde{F}) \leqslant \varepsilon_{0}, \quad\|\tilde{L}, \tilde{F}\|_{C^{2}} \leqslant K,
$$

alors, pour tout $\lambda \geqslant \lambda_{0}(K)$, tout $(f, g) \in L^{2}(\tilde{\Omega}) \times L^{2}\left(\mathbf{R}^{2}\right)$ il existe une unique solution $(u, \phi) \in L^{2}(\tilde{\Omega}) \times H^{1}\left(\mathbf{R}^{2}\right)$ de (6.2.5). En outre, $\gamma u \in L^{2}\left(\mathbf{R}^{2}\right)$ et pour tout $T \in \mathbf{R}$ on $a$

$$
\left(\lambda\|u\|_{0, T}^{2}+\|\gamma u\|_{0, T}^{2}+\lambda^{2}\|\phi\|_{0, T}^{2}\right) \leqslant C_{0}\left\{\lambda^{-1}\|f\|_{0, T}^{2}+\|g\|_{0, T}^{2}\right\} .
$$

Si f et $g$ sont nulles pour $t \leqslant t_{0}$ il en est de même de $(u, \phi)$.

Enfin, si les coefficients de $L$ et $\tilde{F}$ sont $C^{\infty}$ et ont toutes leurs dérivées bornées et si $f$ et $g$ sont $C^{\infty}$ à support compact dans $\overline{\tilde{\Omega}}$ et $\mathbf{R}^{2}$, la solution $(u, \phi)$ est dans $C^{\infty}(\overline{\tilde{\Omega}}) \times$ $C^{\infty}\left(\mathbf{R}^{2}\right)$.

Dans (6.2.7) \|\|$_{k, T}$ désigne la norme $H^{k}\left(\tilde{\Omega}_{T}\right)$ ou $H^{k}\left(\tilde{\omega}_{T}\right)$ si

$$
\left.\tilde{\Omega}_{T}=\right]-\infty, T[\times] 0,1\left[\times \mathbf{R} \text { et } \tilde{\omega}_{T}=\right]-\infty, T[\times \mathbf{R}
$$

Aux hypothèses de réguluarité des coefficients près, ce théorème résulte immédiatement du travail de A. Majda. Néanmoins, il a paru intéressant d'en donner une démonstration qui utilise des méthodes différentes: on la trouvera dans l'appendice B.

6.3. Inégalités $d$ 'énergie. Nous revenons à la démonstration du Théorème 6.1.1, en commençant par établir des inégalités d'énergie. 
Proposition 6.3.1. Sous les hypothèses du Théorème 6.1.1, il existe $\varepsilon_{0}>0, \lambda_{0}(K)$ et $C_{0}(K)$ tels que, si l'on a (6.1.5), alors, pour tout $\lambda \geqslant \lambda_{0}(K)$ tout $T \leqslant T_{0}$ et tout $(u, \phi) \in \dot{C}^{\infty}\left(\Omega_{T}\right) \times \dot{C}^{\infty}\left(\omega_{T}\right)$, on $a$

$$
\|u, \phi\|_{0, \lambda, T} \leqslant C_{0}(K)\|L u, F(\gamma u, \phi)\|_{0, \lambda, T}^{\prime}
$$

Démonstration. Puisque $\underline{A}+\underline{B}$ est inversible, en supposant $\varepsilon_{0}$ assez petit et en multipliant $L$ par $(A+B)^{-1}$, on se ramène au cas où $A+B=$ Id.

Avec les notations du paragraphe 5.2 , nous posons $\hat{u}=J_{\lambda} u, \hat{\phi}=J_{\lambda+1} \phi$; on a alors $J_{\lambda-1} L u=\hat{L}_{\lambda} \hat{u}$ et $J_{\lambda} F(\gamma u, \phi)=\hat{F}_{\lambda}(\gamma \hat{u}, \hat{\phi})$ avec

$$
\begin{array}{r}
\hat{L}_{\lambda} \hat{u}=t \frac{\partial \hat{u}}{\partial t}+\lambda \hat{u}+[(1-\theta) \hat{A}-\theta \hat{B}] \frac{\partial \hat{u}}{\partial \theta}+t \hat{C} \frac{\partial \hat{u}}{\partial z}, \\
\hat{F}_{\lambda}(\gamma \hat{u}, \hat{\phi})=\left[t \frac{\partial \hat{\phi}}{\partial t}+(\lambda+1) \hat{\phi}\right] l+t \frac{\partial \hat{\phi}}{\partial z} p+\hat{\phi} q+m \gamma \hat{u},
\end{array}
$$

$\gamma \hat{u}$ désignant les traces $\gamma^{\prime} \hat{u}(t, z)=\hat{u}(t, 0, z), \gamma^{\prime \prime} \hat{u}(t, z)=\hat{u}(t, 1, z) ; \hat{A}(t, \theta, z)=$ $A(t \theta, t(1-\theta), z)$ et des formules analogues pour $\hat{B}$ et $\hat{C}$.

Avec la définition (5.2.2), posons

$$
\begin{gathered}
\|v, \Psi\|_{0, \lambda, T}^{\prime \prime}=\left\{\lambda\|v\|_{0, \lambda, T}^{\prime \prime 2}+\|\gamma v\|_{0, \lambda, T}^{\prime 2}+\|\Psi\|_{1, \lambda, T}^{\prime 2}\right\}^{1 / 2}, \\
\|f, g\|_{0, \lambda, T}^{\prime \prime}=\left\{\lambda^{-1}\|f\|_{0, \lambda, T}^{\prime \prime 2}+\|g\|_{0, \lambda, T}^{\prime \prime 2}\right\}^{1 / 2} .
\end{gathered}
$$

Compte tenu du Lemme 5.2.1 l'estimation d'énergie, (6.3.1) équivaut à la suivante

$$
\|v, \Psi\|_{0, \lambda, T}^{\prime \prime} \leqslant C_{1}(K)\left\|\hat{L}_{\lambda} v, \hat{F}_{\lambda}(\gamma v, \Psi)\right\|_{0, \lambda, T}^{\prime \prime}
$$

pour $(v, \Psi) \in \dot{C}^{\infty}\left(\hat{\Omega}_{T}\right) \times \dot{C}^{\infty}\left(\omega_{T}\right), \dot{C}^{\infty}\left(\hat{\Omega}_{T}\right)$ désignant l'espace des fonctions $C^{\infty}$ à support compact dans $] 0, T[\times[0,1] \times \mathbf{R}$.

Pour démontrer l'estimation (6.3.5) nous appliquons aux fonctions $v$ et $\Psi$ les découpages (5.3.4) et (5.3.13). On écrit

$$
\begin{aligned}
\hat{L}_{\lambda} v_{j} & =\chi\left(2^{j} t\right) \hat{L}_{\lambda} v+w_{j}, \\
\hat{F}_{\lambda}\left(\gamma v_{j}, \phi_{j}\right) & =\chi\left(2^{j} t\right) \hat{F}_{\lambda}\left(\gamma v, \phi_{j}\right)+g_{j},
\end{aligned}
$$

avec

$$
\left\|w_{j}\right\|_{0, \lambda, T}^{\prime \prime} \leqslant C\|v\|_{L^{2}\left[2^{-j-1} \leqslant t \leqslant 2^{-j+1}\right]}, \quad\left\|g_{j}\right\|_{0, \lambda, T}^{\prime \prime} \leqslant C\|\phi\|_{L^{2}\left[2^{-j-1} \leqslant t \leqslant 2^{-j+1}\right]} .
$$

Avec le Lemme 5.2.2 on obtient donc les majorations

$$
\|v, \Psi\|_{0, \lambda, T}^{\prime \prime 2} \leqslant C \sum_{j}\left\|v_{j}, \Psi_{j}\right\|_{0, \lambda, T}^{\prime 2}
$$

$$
\begin{aligned}
& \sum\left\|\hat{L}_{\lambda} v_{j}, \hat{F}_{\lambda}\left(\gamma v_{j}, \phi_{j}\right)\right\|_{0, \lambda, T}^{\prime \prime 2} \\
& \quad \leqslant C\left\{\left\|\hat{L}_{\lambda} v, \hat{F}_{\lambda}(\gamma v, \Psi)\right\|_{0, \lambda, T}^{\prime \prime 2}+\lambda^{-1}\|v\|_{0, \lambda, T}^{2}+\|\phi\|_{0, \lambda, T}^{2}\right\} .
\end{aligned}
$$

Quitte à augmenter $\lambda_{0}(K)$, on voit donc qu'il suffit d'établir (6.3.5) pour les fonctions $\left(v_{j}, \Psi_{j}\right)$. 
Nous appliquons à ces fonctions les dilatations (5.2.7) et (5.2.14) on écrit alors que

$$
\widehat{L_{\lambda} v_{j}}=\tilde{L}_{\lambda}^{j} \tilde{v}_{j}, \quad \hat{F}_{\lambda}\left(\gamma v_{j}, \Psi_{j}\right)=\tilde{F}_{\lambda}^{j}\left(\gamma \tilde{v}_{j}, \Psi_{j}\right)
$$

avec

$$
\begin{gathered}
\tilde{L}_{\lambda}^{j} w=t \frac{\partial w}{\partial t}+\lambda w+\left[(1-\theta) \tilde{A}_{j}-\theta \tilde{B}_{j}\right] \frac{\partial w}{\partial \theta}+t \tilde{C}_{j} \frac{\partial w}{\partial z}, \\
\tilde{F}_{\lambda}^{j}(\gamma w, \chi)=\left(t \frac{\partial \chi}{\partial t}-(\lambda+1) \chi\right) \tilde{l}_{j}+t \frac{\partial \chi}{\partial z} \tilde{p}_{j}+\chi \tilde{q}_{j}+\tilde{m}_{j} \gamma w
\end{gathered}
$$

et $\tilde{A}_{j}(t, \theta, z)=\hat{A}\left(2^{-j} t, \theta, 2^{-j} z\right), l_{j}(t, z)=\tilde{l}\left(2^{-j} t, 2^{-j} z\right)$, etc.

Avec le Lemme 5.2.3 on voit que la Proposition 6.3.1 résulte alors des inégalités suivantes:

$$
\begin{aligned}
& \lambda\|w\|_{0, T_{j}}^{2}+\|\gamma w\|_{0, T_{j}}^{2}+\|\chi\|_{1, T_{j}}^{2}+\lambda^{2}\|\chi\|_{0, T_{j}}^{2} \\
& \quad \leqslant C_{2}(K)\left\{\lambda^{-1}\left\|L_{\lambda}^{j} w\right\|_{0, T_{j}}^{2}+\left\|\tilde{F}_{\lambda}^{j}(\gamma w, \chi)\right\|_{0, T_{j}}^{2}\right\} .
\end{aligned}
$$

avec $C_{2}(K)$ indépendant de $j, T \leqslant T_{0}, \lambda \geqslant \lambda_{2}(K)$, pour $w$ [resp. $\chi$ ] $C^{\infty}$ sur ]0, $\left.T_{j}\right] \times[0,1] \times \mathbf{R}$ [resp. $\left.] 0, T_{j}\right] \times \mathbf{R}$ ] à support compact dans $\frac{1}{2} \leqslant t \leqslant T_{j}$.

Les coefficients de $\tilde{L}_{\lambda}^{j}$ et $\tilde{F}_{\lambda}^{j}$ sont de classe $C^{2}$ sur $\left[\frac{1}{2}, 2\right] \times[0,1] \times \mathbf{R}$, et bornés uniformément par rapport à $j$. En prolongeant ces coefficients et en effectuant le changement de variable $t=e^{s}$ on se ramène à un système de la forme (6.2.1), (6.2.2) et l'estimation (6.3.9) résulte du Théorème 6.2.1.

Proposition 6.3.2. Sous les hypothèses du Théorème 6.1.1 il existe $\varepsilon_{0}>0, \lambda_{0}(K)$ et $C_{0}(K)$ tels que si l'on a (6.1.5) alors pour tout $\lambda \geqslant \lambda_{0}(K)$, tout $T \leqslant T_{0}, k \leqslant N$ et tout $(u, \phi) \in \dot{C}^{\infty}\left(\Omega_{T}\right) \times \dot{C}^{\infty}\left(\omega_{T}\right)$ :

$$
\|u, \phi\|_{k, \lambda, T} \leqslant C_{0}(K)\|L u, F(\gamma u, \phi)\|_{k, \lambda, T}^{\prime} .
$$

PreUve. La démonstration se fait par récurrence sur $k$ et nous supposons (6.3.10) établi à l'ordre $k<N$.

Notons $D_{1}=x \partial_{x}+y \partial_{y}$ et $D_{2}=(x+y) \partial_{z}$. $\left[L, D_{i}\right]$ est une somme de termes de la forme $a \partial, x(\partial a) \partial^{\prime}, y(\partial a) \partial^{\prime}$ où $\partial$ et $\partial^{\prime}$ désignent des dérivations d'ordre 1 et a une fonction de la forme $a=\underline{a}+a_{1}$, avec $\underline{a}$ constante et $a_{1} \in H^{N}\left(\Omega_{T_{0}}\right)$.

Pour $u \in H_{\lambda+1 / 2}^{k+1}\left(\Omega_{T}\right)$ on a $\partial u \in H_{\lambda-1 / 2}^{k}\left(\Omega_{T}\right)$, et on en déduit, avec la Proposition 5.3.1, que

$$
\left\|\left[L, D_{i}\right] u\right\|_{k, \lambda-1 / 2, T} \leqslant C\|u\|_{k+1, \lambda+1 / 2, T} .
$$

Notons de même $D_{1}=t \partial_{t}$ et $D_{2}=t \partial_{z}$, et remarquons que $\gamma D_{i} u=D_{i} \gamma u$. Alors, grâce à la Proposition 5.3.1, on montre aussi que

$$
\left\|F\left(\gamma D_{i} u, D_{i} \phi\right)-D_{i} F(\gamma u, \phi)\right\|_{k, \lambda, T} \leqslant C\left\{\|\gamma u\|_{k, \lambda, T}+\|\phi\|_{k+1, \lambda, T}\right\} .
$$

On tire de (6.3.11), (6.3.12) et de l'hypothèse de récurrence (6.3.10) que

$$
\left\|D_{i} u, D_{i} \phi\right\|_{k, \lambda, T} \leqslant C\left\{\|L u, F(\gamma u, \phi)\|_{k+1, \lambda, T}^{\prime}+\lambda^{-1}\|u, \phi\|_{k+1, \lambda, T}\right\} .
$$


Admettons un instant les estimations

$$
\|\phi\|_{k+1, \lambda+1, T} \leqslant C\left\{\sum_{i=1,2}\left\|D_{i} \phi\right\|_{k, \lambda+1, T}+\lambda\|\phi\|_{k, \lambda+1, T}\right\} .
$$

$$
\|u\|_{k+1, \lambda+1 / 2, T} \leqslant C\left\{\sum_{i=1,2}\left\|D_{i} u\right\|_{k, \lambda+1 / 2, T}+\lambda\|u\|_{k, \lambda+1 / 2, T}+\|L u\|_{k, \lambda-1 / 2, T}\right\} .
$$

Avec (6.3.13) on en déduit que:

$$
\begin{aligned}
\|u, \phi\|_{k+1, \lambda, T} \leqslant C\left\{\|L u, F(\gamma u, \phi)\|_{k+1, \lambda, T}+\lambda\|u, \phi \mid\|_{k, \lambda, T}\right. \\
\left.+\frac{1}{\lambda}\|(u, \phi)\|_{k+1, \lambda, T}\right\} .
\end{aligned}
$$

Pour $\lambda$ assez grand, le dernier terme de (6.3.16) s'absorbe dans le membre de gauche; le terme $\lambda\|\| u, \phi \mid \|_{k, \lambda, T}$ s'estime par l'hypothè̀se de récurrence, et on en déduit immédiatement l'estimation 6.3.10 à l'ordre $k+1$.

Il nous reste à établir (6.3.14) et (6.3.15); (6.3.14) est une conséquence quasiimmédiate des définitions. Pour obtenir (6.3.15), on introduit l'opérateur $D_{3}$ $=(x+y)\left(\partial_{x}-\partial_{y}\right)$ et on remarque d'abord que

$$
\|u\|_{k+1, \lambda+1 / 2, T} \leqslant C\left\{\sum_{i=1}^{3}\left\|D_{i} u\right\|_{k, \lambda+1 / 2, T}+\lambda\|u\|_{k, \lambda+1 / 2, T}\right\}
$$

comme on le voit immédiatement en passant en coordonnées cylindriques, et en utilisant le Lemme 5.2.1, puisque dans ces coordonnées les opérateurs $D_{i}$ deviennent $t \partial_{t}, t \partial_{z}$ et $\partial_{\theta}$.

Maintenant on écrit que

$$
(x+y) L=(A+B) D_{1}+\left(A-\frac{x}{x+y}(A+B)\right) D_{3}+C D_{2} .
$$

D'apres l'hypothèse $(\alpha)$ du paragraphe 3.3 , si $\varepsilon_{0}$ est assez petit, la matrice $A(X)-\theta(A+B)(X)$ est inversible pour $\theta \in[0,1]$ et $X \in \bar{\Omega}_{T_{0}}$; notons $G(\theta ; X)$ son inverse et $G^{\prime}(x, y, z)=G(x /(x+y) ; x, y, z)$. On remarque que $G(\theta, X)$ est de la forme $\underline{G}(\theta)+G_{1}(\theta, X)$ où $\underline{G}$ est $C^{\infty}$ sur $[0,1]$ et $G_{1}$ une application $C^{\infty}$ de $\theta \in[0,1]$ à valeurs dans $H^{N}\left(\Omega_{T_{0}}\right)$. On a

$$
D_{3} u=G^{\prime}\left\{(x+y) L u-(A+B) D_{1} u-C D_{2} u\right\}
$$

et on tire des Propositions 5.3.1 et 5.3.5, l'estimation

$$
\left\|D_{3} u\right\|_{k, \lambda+1 / 2, T}<C\left\{\|L u\|_{k, \lambda-1 / 2, T}+\sum_{i=1,2}\left\|D_{i} u\right\|_{k, \lambda+1 / 2, T}\right\} .
$$

Avec (6.3.17), on en déduit la majoration (6.3.15) ce qui achève la démonstration de la Proposition 6.3.2.

6.4. Démonstration du Théorème 6.1.1. Nous montrons d'abord l'existence d'une solution pour le problème (6.1.2), nous établirons l'unicité ensuite. 
(1) Existence.

LeMme 6.4.1. Il existe $\varepsilon_{0}>0$ tel que, si $(L, F)$ est un système (6.1.1) dont les coefficients sont $C^{\infty}$ et on toutes leurs dérivées bornées sur $\bar{\Omega}_{T_{0}}$ et $\bar{\omega}_{T_{0}}$, et qui vérifie $\varepsilon(L, F) \leqslant \varepsilon_{0}$ alors pour tout $(f, g) \in \dot{C}^{\infty}\left(\Omega_{T}\right) \times \dot{C}^{\infty}\left(\omega_{T}\right)$ le problème (6.1.2) possède une solution $(u, \phi) \in H^{\infty}\left(\Omega_{T}\right) \times H^{\infty}\left(\omega_{T}\right)$, nulle au voisinage de l'arête $x=y=0$, $t=0$.

Preuve. Passons en coordonnées polaires et posons $\hat{f}=J_{0} f, \hat{g}=J_{1} g$. Avec la notation (6.3.2), le problème (6.1.2) s'écrit

$$
\hat{L}_{1} \hat{u}=\hat{f}, \quad \hat{F}_{1}(\gamma \hat{u}, \hat{\phi})=\hat{g},
$$

$\hat{f}$ et $\hat{g}$ sont supportés dans $t \geqslant t_{0}>0$. On effectue le changement de variables $t=e^{s}$, et 0 , se ramène à un système du type (6.2.1), (6.2.2):

$$
\tilde{L} \tilde{u}=\tilde{f}, \quad \tilde{F}(\gamma \tilde{u}, \tilde{\phi})=\tilde{g} .
$$

On prolonge les coefficients de $\tilde{L}$ et $\tilde{F}$ pour $s \leqslant \log t_{0}$ et $s \geqslant \log T$, et le Théorème 6.2 .2 fournit une solution $(\tilde{u}, \tilde{\phi}) \in C^{\infty}(\tilde{\tilde{\Omega}}) \times C^{\infty}(\tilde{\mathbf{R}})$, nulle pour $s \leqslant \log t_{0}$. En revenant aux coordonnées initiales, on en déduit une solution $(u, \phi)$ du problème (6.1.2).

Lemme 6.4.2. Il existe $\varepsilon_{0}, \lambda_{0}(K)$ et $C_{0}(K)$ tels que si le système $(L, F)$ vérifie (6.1.5) et a ses coefficients $C^{\infty}$ bornés, ainsi que leurs dérivées sur $\bar{\Omega}_{T_{0}} \times \bar{\omega}_{T_{0}}$, alors pour $\lambda \geqslant \lambda_{0}(K), T \leqslant T_{0},(f, g) \in W_{\lambda, T}^{\prime N}$, le problème (6.1.2) possède une solution $(u, \phi) \in W_{\lambda, T}^{N}$, qui vérifie l'inégalité d'énergie 6.3.10.

Preuve. $\dot{C}^{\infty}\left(\Omega_{T}\right)$ et $\dot{C}^{\infty}\left(\omega_{T}\right)$ sont denses dans $H_{\lambda-1 / 2}^{k}\left(\Omega_{T}\right)$ et $H_{\lambda}^{k}\left(\omega_{T}\right)$. On approche alors $(f, g)$ par une suite $\left(f_{j}, g_{j}\right) \in \dot{C}^{\infty}\left(\Omega_{T}\right) \times \dot{C}^{\infty}\left(\omega_{T}\right)$ et le Lemme 6.4.1 permet de trouver $\left(u_{j}, \phi_{j}\right)$ tels que

$$
L u_{j}=f_{j}, \quad F\left(\gamma u_{j}, \phi_{j}\right)=g_{j},
$$

et $\left(u_{j}, \phi_{j}\right) \in W_{\mu, T}^{l}$ pour tout $l$ et tout $\mu$. L'inégalité (6.3.10) se prolonge par continuité à $H_{\lambda+1 / 2}^{N+1}\left(\Omega_{T}\right) \times H_{\lambda+1}^{N+1}\left(\omega_{T}\right)$, et on en déduit que

$$
\left\|\left(u_{j}, \phi_{j}\right)\right\|_{N, \lambda, T} \leqslant C\|f, g\|_{N, \lambda, T} .
$$

Quitte à extraire une sous suite, on en déduit que $\left(u_{j}, \phi_{j}\right)$ converge vers une limite $(u, \phi)$, faiblement dans $W_{\lambda, T}^{N}$, et fortement dans $C^{1}\left(\bar{\Omega}_{T}\right) \times C^{1}\left(\bar{\omega}_{T}\right)$. Le Lemme 6.4.2 s'en déduit par passage à la limite dans (6.4.3) et (6.4.4).

LEMME 6.4.3. On peut supprimer dans le Lemme 6.4.2, l'hypothèse de régularité $C^{\infty}$ des coefficients.

Preuve. On approche les coefficients de $(L, F)$ par des coefficients $C^{\infty}$, construisant des systèmes $\left(L_{j}, F_{j}\right)$. Pour $j$ assez grand on a

$$
\varepsilon\left(L_{j}, F_{j}\right) \leqslant 2 \varepsilon(L, F), \quad\left\|L_{j}, F_{J}\right\|_{N} \leqslant\|L, F\|_{N}+1 .
$$

Pour $(f, g) \in W_{\lambda, T}^{\prime N}$, si $\varepsilon(L, F)$ est assez petit, le Lemme 6.4 .3 fournit une solution $\left(u_{j}, \phi_{j}\right)$ au problème

$$
L_{j} u_{j}=f, \quad F_{j}\left(\gamma u_{j}, \phi_{J}\right)=g
$$


En même temps, pour $\lambda$ assez grand on a

$$
\left\|u_{j}, \phi_{j}\right\|_{N, \lambda, T} \leqslant C\|f, g\|_{N, \lambda, T}^{\prime}
$$

avec $C$ indépendant de $j$. Le Lemme 6.4 .3 se déduit à nouveau par passage à la limite dans (6.4.6) (6.4.7).

Le Lemme 6.4.3 achève la démonstration de la partie existence du Théorème 6.1.1.

(2) Unicité. Notons $\mathscr{D}_{\lambda, T}$ l'espace des $(u, \phi) \in L_{\lambda+1 / 2}^{2}\left(\Omega_{T}\right) \times H_{\lambda+1}^{1}\left(\omega_{T}\right)$ tels que $L u \in L_{\lambda-1 / 2}^{2}\left(\Omega_{T}\right)$ et $\gamma u \in L_{\lambda}^{2}\left(\omega_{\text {) }}\right.$ (on notera que si $u \in L_{\lambda+1 / 2}^{2}$ et $L u \in L_{\lambda-1 / 2}^{2}$ les $\gamma u$ sont bien définies).

Notons aussi $\dot{\mathscr{D}}_{\lambda . \mathrm{T}}$ le sous-espace des fonctions à support compact dans $\bar{\Omega}_{T}$ et $\bar{\omega}_{T}$ et nulles au voisinage d l'arête $x=y=0$ et $t=0$.

Par troncature, il est clair $\dot{\mathscr{D}}_{\lambda, T}$ est dense dans $\mathscr{D}_{\lambda, T}$. En dehors d l'arête les solutions faibles sont solutions fortes, et on en déduit que $W_{\lambda, T}^{1}$ est dense dans $\mathscr{D}_{\lambda, T}$.

L'inégalité d'énergie (6.3.1) se prolonge donc à $W_{\lambda, T}^{1}$ puis à $\mathscr{D}_{\lambda, T}$, ce qui assure l'unicité dans $\mathscr{D}_{\lambda, T}$ de la solution du problème (6.2.1).

7. Le problème de Goursat non linéaire. Dans cette partie nous donnons la démonstration du Théorème 3.3.1. On procède en deux étapes: on construit d'abord une solution approchée au sens du développement de Taylor sur l'arête, puis, par un schéma itératif basé sur le Théorème 6.1.1, on construit une solution exacte.

7.1. Solution approchée-choix des paramètres. On utilise les notations du paragraphe 3.3, et on considère un système (3.3.3) vérifiant les hypothèses indiquées.

Si $\phi$ est définie sur $\omega$, il sera commode de noter

$$
j \phi(X)=\left(\tilde{\phi}(X),\left(\frac{\tilde{\phi}}{t}\right)(X), \widetilde{\nabla \phi}(x), x\left(\widetilde{\nabla \frac{\phi}{t}}\right)(X), y\left(\widetilde{\nabla \frac{\phi}{t}}\right)(X)\right)
$$

avec la convention (3.1.1), de sorte que les coefficients $A(u, \phi)$ de $L$ sont des fonctions $C^{\infty}$ au voisinage de l'origine de $x, u(x), j \phi(x)$. On introduit alors l'espace $W_{T}$ des $(u, \phi) \in H^{N}\left(\Omega_{T}\right) \times N^{N+1}\left(\omega_{T}\right)$ tels que $\gamma u \in H^{N}\left(\omega_{T}\right)$ et $j \phi \in H^{N}\left(\Omega_{T}\right)$, muni de la norme

$$
\|u, \phi\|_{W_{T}}=\|u\|_{H^{N}\left(\Omega_{T}\right)}+\|\gamma u\|_{H^{N}\left(\omega_{T}\right)}+\|\phi\|_{H^{N+1}\left(\omega_{T}\right)}+\|j \phi\|_{H^{N}\left(\Omega_{T}\right)}
$$

On notera aussi

$$
\varepsilon_{T}(u, \phi)=\|u\|_{L^{\infty}\left(\Omega_{T}\right)}+\|\phi\|_{L^{\infty}\left(\omega_{T}\right)}+\|\nabla \phi\|_{L^{\infty}\left(\omega_{T}\right)}+\|j \phi\|_{L^{\infty}\left(\Omega_{T}\right)} .
$$

On se donne maintenant une fonction $\chi \in C_{0}^{\infty}(\mathbf{R})$, qui vaut 1 pour $|z| \leqslant \frac{1}{2}$ et nulle pour $|z| \geqslant 1$. Pour $\rho>0$ on note

$$
\begin{aligned}
A^{\rho}(u, \phi) & =A(x, y, z \chi(z / \rho), u, j \phi), \\
\mathscr{F}^{\rho}(\gamma u, \phi) & =\mathscr{F}(t, z \chi(z / \rho), \gamma u, \nabla \phi, \phi),
\end{aligned}
$$

et $L^{\rho}(u, \phi)$ l'opérateur $A^{\rho}(u, \phi)(\partial / \partial x)+B^{\rho}(u, \phi)(\partial / \partial y)+C^{\rho}(u, \phi)(\partial / \partial z)$, de sorte qu'il nous suffit de résoudre pour $\rho$ assez petit

$$
L^{\rho}(u, \phi)=0, \quad \mathscr{F}^{\rho}(u, \phi)=0 ; \quad \phi_{\mid t=0}=0 .
$$


Nous noterons $F_{\gamma u, \phi}^{\rho}$ le système obtenu par différentiation de $\mathscr{F}^{\rho}$ autour de $(\gamma u, \phi)$

$$
F_{\gamma u, \phi}^{\rho}(\gamma v, \Psi)=\left.\frac{d}{d s}\right|_{s=0} \mathscr{F}^{\rho}(\gamma u+s \gamma v, \phi+s \Psi),
$$

et on écrira

(7.1.6)

$$
F_{\gamma u, \phi}^{\rho}(\gamma v, \Psi)=l^{\rho}(\gamma u, \phi) \frac{\partial \Psi}{\partial t}+p^{\rho}(\gamma u, \phi) \frac{\partial \Psi}{\partial z}+q^{\rho}(\gamma u, \phi) \Psi+m^{\rho}(\gamma u, \phi) \cdot \gamma v .
$$

Pour résoudre (7.1.5) nous étudierons les problèmes linéarisés suivants:

$$
L^{\rho}(u, \phi) v=f, \quad F_{\gamma u, \phi}^{\rho}(\gamma v, \Psi)=g ; \quad \Psi_{\mid t=0}=0,
$$

qui sont du type (6.1.1). En particulier, si on fige à l'origine les coefficients du linéarisé autour de $\gamma u=0, \phi=0$, on obtient le système $(\underline{L}, \underline{F})$ introduit en (3.3.6) (3.3.7).

Pour construire la solution approchée, nous ferons usage des systèmes

$$
\left\{\begin{array}{c}
L_{0}^{\rho}(u, \phi) v=A^{\rho}(u, \phi) \frac{\partial v}{\partial x}+B^{\rho}(u, \phi) \frac{\partial v}{\partial y}, \\
F_{0(\gamma u, \phi)}^{\rho}(\gamma v, \Psi)=l^{\rho}(u, \phi) \frac{\partial \Psi}{\partial t}+m^{\rho}(u, \phi) \gamma v,
\end{array}\right.
$$

qui sont du type (4.1.1) (4.1.2), si l'on considère la variable $z$ come paramètre. De même on note

$$
\underline{L}_{0} v=\underline{A} \frac{\partial v}{\partial x}+\underline{B} \frac{\partial v}{\partial y}, \quad \underline{F_{0}}(\gamma v, \Psi)=\underline{l} \frac{\partial \Psi}{\partial t}+\underline{m} \gamma v .
$$

Le Théorème 4.1 .1 associe au système $\left(L_{0}, F_{0}\right)$ un réel $\varepsilon_{0}>0$, alors que le Théorème 6.1.1 associe à $(\underline{L}, \underline{F})$ un $\varepsilon_{0}>0$ et des fonctions $\lambda_{0}(K), C_{0}(K)$. On peut bien sûr prendre le même $\varepsilon_{0}$.

La forme (7.1.4) des coefficients de $L^{\rho}$ et $F^{\rho}$, montre que

LEMME 7.1.1. Il existe $\rho^{*}>0, \varepsilon^{*}>0$ et $T_{0}>0$ tels que pour tout $(u, \phi) \in W_{T_{0}}$ vérifiant

$$
\varepsilon_{T_{0}}(u, \phi) \leqslant 2 \varepsilon^{*}
$$

alors, pour $\rho \leqslant \rho^{*}$ on $a$ :

$$
\varepsilon\left(L^{\rho}(u, \phi), F_{\gamma u, \phi}^{\rho}\right) \leqslant \varepsilon_{0}, \quad \varepsilon\left(L_{0}^{\rho}(u, \phi), F_{0,(\gamma u, \phi)}^{\rho}\right) \leqslant \varepsilon_{0} .
$$

En outre il existe une fonction $K$, continue sur $] 0, \rho^{*}[\times[0, \infty[$, telle que

$$
\left\|L^{\rho}(u, \phi), F_{\gamma u, \phi}^{\rho}\right\|_{N} \leqslant K\left(\rho,\|u, \phi\| w_{T_{0}}\right) .
$$

Dans ce lemme sont utilisées les notations $\varepsilon(L, F)$ et $\|L, F\|_{N}$ introduites aux paragraphes 4.1 et 6.1 .

Proposition 7.1.2. Pour tout $\varepsilon>0$, il existe des constantes $\left.\rho \in] 0, \rho^{*}\right]$ et $M$, et une suite $\left(u, \phi_{j}\right) \in C^{\infty}(\bar{\Omega}) \times C^{\infty}(\bar{\omega})$ à support dans $\bar{\Omega}_{T_{0 . \rho}}$ et $\bar{\omega}_{T_{0 . \rho}}$ et telles que

$$
\begin{cases}f_{j}=L^{\rho}\left(u_{j}, \phi_{j}\right)=O\left((x+y)^{j}\right) & (\text { pour } x+y \rightarrow 0), \\ g_{j}=\mathscr{F}^{\rho}\left(u_{j}, \phi_{j}\right)=O\left(t^{j+1}\right) & (\text { pour } t \rightarrow 0),\end{cases}
$$




$$
\begin{gathered}
\varepsilon_{T_{0}}\left(u_{j}, \phi_{j}\right) \leqslant \varepsilon \\
\left\|u_{j}, \phi_{j}\right\|_{w_{T_{0}}} \leqslant M .
\end{gathered}
$$

Preuve. Considérons la fonction qui à $\left(z, v, \tau^{\prime}, \tau^{\prime \prime}\right) \in \mathbf{R} \times \mathbf{R}^{2} \times \mathbf{R} \times \mathbf{R}$ associe $\left(\mathscr{F}^{\prime}\left(0, z, v,\left(\tau^{\prime}, 0\right), 0\right), \mathscr{F}^{\prime \prime}\left(0, z, v,\left(\tau^{\prime \prime}, 0\right), 0\right)\right) \in \mathbf{R}^{4}$. Sa différentielle à l'origine par rapport aux variables $(v, \tau)$ est

$$
(\delta v, \delta \tau) \rightarrow\left(\delta \tau^{\prime} \cdot \underline{l}^{\prime}+\underline{m}^{\prime} \delta v, \delta \tau^{\prime \prime} \underline{l}^{\prime \prime}+\underline{m}^{\prime \prime} \delta v\right) .
$$

Il résulte de l'hypothèse $(\gamma)$ que cette différentielle est bijective et, avec (3.3.5), le théorème des fonctions implicites permet de trouver des fonctions $v(z)$ et $\tau(z), C^{\infty}$ au voisinage de l'origine, et telles que

$$
\begin{aligned}
& \mathscr{F}^{\prime}\left(0, z, v(z),\left(\tau^{\prime}(z), 0\right), 0\right)=0, \\
& \mathscr{F}^{\prime \prime}\left(0, z, v(z),\left(\tau^{\prime \prime}(z), 0\right), 0\right)=0, \\
& v(0)=0 ; \quad \tau(0)=0 .
\end{aligned}
$$

Si $\rho$ est assez petit, les fonctions

$$
u_{0}(x, y, z)=v(z \chi(z / \rho)), \quad \phi_{0}(t, z)=t \tau(z \chi(z / \rho)),
$$

sont définies et $C^{\infty}$ sur $\bar{\Omega}$ et $\bar{\omega}$, vérifient

$$
\varepsilon_{T_{0}}\left(u_{0}, \phi_{0}\right) \leqslant \varepsilon
$$

et (7.1.15) implique que

$$
\mathscr{F}^{\rho}\left(u_{0}, \phi_{0}\right)_{\mid t=0}=0 .
$$

Pour simplifier notons $L_{0}=L_{0}^{\rho}\left(u_{0}, \phi_{0}\right), F_{0}=F_{0,\left(\gamma u_{0}, \phi_{0}\right)}^{\rho}$. On peut toujours supposer que $\varepsilon \leqslant \varepsilon^{*}$ et $\rho \leqslant \rho^{*}$, si bien que $\varepsilon\left(L_{0}, F_{0}\right) \leqslant \varepsilon_{0}$, d'après le lemme 7.1.1. Le Théorème 4.1.1 permet alors de définir par récurrence une suite $\left(u_{n}, \phi_{n}\right)$ de la manière suivante:

$$
\begin{aligned}
u_{n+1} & =u_{n}+v_{n}, \quad \phi_{n+1}=\phi_{n}+\Psi_{n}, \\
L_{0} v_{n} & =-L^{\rho}\left(u_{n}, \phi_{n}\right) u_{n}, \\
F_{0}\left(\gamma v_{n}, \Psi_{n}\right) & =-\mathscr{F}^{\rho}\left(\gamma u_{n}, \phi_{n}\right) ; \quad \Psi_{n \mid t=0}=0 .
\end{aligned}
$$

On obtient immédiatement par récurrence que $\left(u_{n}, \phi_{n}\right) \in C^{\infty}\left(\bar{\Omega}_{T_{0}}\right) \times C^{\infty}\left(\bar{\omega}_{T_{0}}\right)$, et que $\left(u_{n}, \phi_{n}\right)=0$ pour $|z| \geqslant \rho$. Montrons par récurrence que (7.1.12) est satisfait et que $u_{n}-u_{0}=O(x+y), \phi_{n}-\phi_{0}=O\left(t^{2}\right)$. Pour $n=0$ cela résulte de (7.1.18). Si cela est vrai à l'ordre $n$, alors, d'après le Théorème 4.1.1 on a

$$
v_{n}=O\left((x+y)^{n+1}\right), \quad \Psi_{n}=O\left(t^{n+2}\right) .
$$

Maintenant on écrit que

$$
f_{n+1}=\left(L\left(u_{n+1}, \phi_{n+1}\right)-L^{\rho}\left(u_{n}, \phi_{n}\right)\right) u_{n+1}+\left(L^{\rho}\left(u_{n}, \phi_{n}\right)-L_{0}\right) v_{n} .
$$

On tire de (7.1.20) que $j \Psi_{n}=O\left((x+y)^{n+1}\right)$, puis que $A^{\rho}\left(u_{n+1}, \phi_{n+1}\right)-$ $A^{\rho}\left(u_{n}, \phi_{n}\right)=O\left((x+y)^{n+1}\right)$. De même $j\left(\phi_{n}-\phi_{0}\right)=O(t)$ et $A^{\rho}\left(u_{n}, \phi_{n}\right)$ $A^{\rho}\left(u_{0}, \phi_{0}\right)=O(x+y)$. Ecrivant que $\nabla_{x, y} v_{n}=O\left((x+y)^{n}\right)$ et $\partial v_{n} / \partial z=$ $O\left((x+y)^{n+1}\right)$ on obtient finalement que $f_{n+1}=O\left((x+y)^{n+1}\right)$. 
De même on écrit que

$$
g_{n+1}=\left(F_{\gamma u_{n}, \phi_{n}}^{\rho}-F_{0}\right)\left(\gamma v_{n}, \Psi_{n}\right)+O\left(\left|\gamma v_{n}\right|^{2}+\left|\Psi_{n}\right|^{2}\right)
$$

et avec (7.1.20) et l'annulation $\gamma u_{n}-\gamma u_{0}=O(t), \phi_{n}-\phi_{0}=O\left(t^{2}\right)$ on en déduit que $g_{n+1}=O\left(t^{n+2}\right)$.

Pour satisfaire à $(7.1 .13),(7.1 .14)$ on modifie la suite $\left(u_{n}, \phi_{n}\right)$, en posant, pour $n>N$,

$$
\begin{aligned}
& u_{n}^{*}=\chi\left(\frac{x+y}{\delta_{N}}\right) u_{N}+\chi\left(\frac{x+y}{\delta_{n}}\right)\left(u_{n}-u_{N}\right), \\
& \phi_{n}^{*}=\chi\left(\frac{t}{\delta_{N}}\right) \phi_{N}+\chi\left(\frac{t}{\delta_{n}}\right)\left(\phi_{n}-\phi_{N}\right),
\end{aligned}
$$

où $\chi \in C_{0}^{x}(\mathbf{R})$ vaut 1 au voisinage de l'origine.

Puisque $u_{N}-u_{0}=O(x+y)$, que $\phi_{N}-\phi_{0}=O\left(t^{2}\right)$ et que

$$
j \phi_{N}^{*}=\chi\left(\frac{x+y}{\delta_{N}}\right) j \phi_{N}+o\left(\mid \widetilde{\phi_{N}} \frac{\widetilde{t}}{t}\right), \quad \nabla \phi_{N}^{*}=\chi\left(\frac{t}{\delta_{N}}\right) \nabla \phi_{N}+O\left(\left|\frac{\phi_{N}}{t}\right|\right),
$$

on voit, avec (7.1.17), que si $\delta_{N}$ est assez petit, on a

$$
\varepsilon\left(u_{N}^{*}, \phi_{N}^{*}\right) \leqslant K_{0} \varepsilon
$$

où $K_{0}$ ne dépend que de la fonction de troncature $\chi$.

Maintenant, on a $u_{n}-u_{N}=O\left((x+y)^{N+1}\right)$ et $\phi_{n}-\phi_{N}=O\left(t^{N+2}\right)$. On peut donc choisir $\delta_{n}$ assez petit pour que

$$
\varepsilon\left(u_{n}^{*}-u_{N}^{*}, \phi_{n}^{*}-\phi_{N}^{*}\right) \leqslant \varepsilon, \quad\left\|u_{n}^{*}-u_{N}^{*}, \phi_{n}^{*}-\phi_{N}^{*}\right\|_{W_{T_{0}}} \leqslant 1 .
$$

(7.1.13) résulte de (7.1.22), (7.1.23), en changeant $\varepsilon$ en $\varepsilon /\left(K_{0}+1\right)$ et (7.1.23) conduit à (7.1.14) en posant $M=1+\left\|u_{N}^{*}, \phi_{N}^{*}\right\|_{W_{T_{0}}}$.

LEMME 7.1.3. Il existe une constante $H$ telle que pour tout $\lambda$, et tout $\phi \in H_{\lambda+1}^{N+1}\left(\omega_{T_{0}}\right)$, on $a j \phi \in H_{\lambda+1 / 2}^{N}\left(\Omega_{T_{0}}\right)$ et

$$
\|j \phi\|_{N, \lambda+1 / 2, T_{0}} \leqslant H\|\phi\|_{N+1, \lambda+1, T_{0}} .
$$

Preuve. On remarque d'abord que l'opérateur (3.3.1) $\phi \rightarrow \tilde{\phi}$ est continu de $H_{\mu}^{k}$ dans $H_{\mu+1 / 2}^{k}$. Pour $\phi \in H_{\lambda+1}^{N+1}$ on a $\nabla \phi \in H_{\lambda}^{N}, \phi / t \in H_{\lambda}^{N+1}$ et $\nabla(\phi / t) \in H_{\lambda-1}^{N}$. Enfin pour $u \in H_{\mu}^{N}\left(\Omega_{T_{0}}\right)$ on a clairement $x u$ et $y u \in H_{\mu+1}^{N}$. La composition de ces différents opérateurs donne le lemme.

En utilisant le plongement trivial $H_{\lambda}^{N} \hookrightarrow H^{N}$ pour $\lambda \geqslant N$, on obtient

Corollaire 7.1.4. Il existe une constante $H_{0}$ telle que pour tout $\lambda \geqslant N$, et tout $(v, \Psi) \in W_{\lambda, T_{0}}^{N}$, on $a(v, \Psi) \in W_{T_{0}}$ et

$$
\|(v, \Psi)\|_{W_{T_{0}}} \leqslant H_{0}\|\| v, \Psi \|_{N, \lambda, T_{0}} .
$$


Nous sommes maintenant en mesure de procéder au

Choix des paramètres. Les constantes $\rho^{*}, \varepsilon^{*}$ et $T_{0}$ sont fixées par le Lemme 7.1.1. La Proposition 7.1.2, appliquée à $\varepsilon=\varepsilon^{*}$ détermine $\rho$, que l'on peut supposer $\leqslant \rho^{*}$, $M$, et une suite $\left(u_{j}, \phi_{j}\right)$ véfrifiant (7.1.13), (7.1.14). Avec la fonction $K$ du Lemme 7.1.1 et la constante $H_{0}$ du Corollaire 7.1.4, posons $K=K\left(\rho, M+H_{0}\right)$. Enfin, à ce $K$, sont associées des constantes $\lambda_{0}=\lambda_{0}(K)$ et $C_{0}=C_{0}(K)$. Fixons maintenant $j \geqslant \operatorname{Max}\left\{\lambda_{0}(K), N\right\}+1$; appelons $\left(u_{a}, \phi_{a}\right)$ la fonctions $\left(u_{j}, \phi_{j}\right)$ et $\lambda=j-1$, on a alors

$$
\begin{gathered}
\varepsilon_{T_{0}}\left(u_{a}, \phi_{a}\right) \leqslant \varepsilon^{*}, \quad\left\|u_{a}, \phi_{a}\right\|_{W_{T_{0}}} \leqslant M, \\
f_{a}=-L^{\rho}\left(u_{a}, \phi_{a}\right) u_{a} \in H_{\lambda+1 / 2}^{N}\left(\Omega_{T_{0}}\right), \\
g_{a}=-\mathscr{F}^{\rho}\left(\gamma u_{a}, \phi_{a}\right) \in H_{\lambda+1}^{N}\left(\omega_{T_{0}}\right) .
\end{gathered}
$$

En outre, ces choix étant faits, on peut résoudre le problème linéairisé (7.1.7) de la manière suivante:

Proposition 7.1.5. Pour tout $(v, \Psi) \in W_{\lambda, T_{0}}^{N}$ vérifiant

$$
\varepsilon_{T_{0}}(v, \Psi) \leqslant \varepsilon^{*}, \quad\|v, \Psi\|_{N, \lambda, T_{0}} \leqslant 1,
$$

et pour tout $(f, g) \in W_{\lambda, T_{0}}^{\prime k}, k \leqslant N$, si l'on pose $u=u_{a}+v$, et $\phi=\phi_{a}+\Psi$, le problème

$$
L^{\rho}(u, \phi) w=f, \quad F_{\gamma u, \phi}^{\rho}(\gamma w, \theta)=g, \quad \theta_{\mid t=0}=0,
$$

possède une solution $(w, \theta) \in W_{\lambda, T_{0}}^{k}$, qui vérifie pour $T \leqslant T_{0}$.

$$
\|w, \theta\|_{k, \lambda, T} \leqslant C_{0}\|f, g\|_{k, \lambda, T}^{\prime} .
$$

Cette proposition résulte en effet du Théorème 6.1.1, que l'on peut appliquer grâce au Lemme 7.1.1 et au Corollaire 7.1.4.

7.2. Schema iteratif. $\rho$ étant fixé, nous noterons maintenant $L, F$ à la place de $L^{\rho}$, $F^{\rho}$, etc. Nous définissons une suite $\left(u_{n}, \phi_{n}\right)$ par le schéma itératif suivant: $\left(u_{0}, \phi_{0}\right)=$ $\left(u_{a}, \phi_{a}\right)$ et

$$
\begin{cases}u_{n}=u_{a}+v_{n}, & v_{n}=E_{T}\left(w_{n}\right)_{\mid \Omega_{T}}, \\ \phi_{n}=\phi_{a}+\Psi_{N}, & \Psi_{n}=E_{T}\left(\theta_{n}\right)_{\mid \omega_{T}}\end{cases}
$$

avec

$$
\left\{\begin{array}{l}
L\left(u_{n}, \phi_{n}\right) w_{n+1}=-L\left(u_{n}, \phi_{n}\right) u_{a}=f_{n}, \\
F_{\gamma u_{n}, \phi_{n}}\left(\gamma w_{n+1}, \theta_{n+1}\right)=-\mathscr{F}\left(\gamma u_{n}, \phi_{n}\right)+F_{\gamma u_{n}, \phi_{n}}\left(\gamma v_{n}, \Psi_{n}\right)=g_{n}, \\
\theta_{n+1_{1,0}}=0
\end{array}\right.
$$

où $T$ est un paramètre à choisir dans $] 0, T_{0}$ [ et $E_{T}$ un opérateur d'extension, introduit au Lemme 5.1.2, opérant de $H_{\lambda}^{k}\left(\Omega_{T}\right)$ dans $H_{\lambda}^{k}\left(\Omega_{T_{0}}\right)$ pour $k \leqslant N+1$, et de $H_{\lambda}^{k}\left(\omega_{T}\right)$ dans $H_{\lambda}^{k}\left(\omega_{T_{0}}\right)$. La construction du Lemme 5.1 .2 montre que l'on peut faire en sorte que $\gamma E_{T} u=E_{T} \gamma u$, et on utilisera le fait que $E_{T}$ opère de $W_{\lambda, T}^{N}$ dans $W_{\lambda, T_{0}}^{N}$, avec une norme majorée par une constante $H_{1}$, indépendante de $T \leqslant T_{0}$ et de $\lambda$. 
Proposition 7.2.1. Il existe $\left.\left.T^{*} \in\right] 0, T_{0}\right]$ tel que, pour $T \leqslant T^{*}$, il existe une suite $\left(u_{n}, \phi_{n}\right)$ vérifiant $(7.2 .1)(7.2 .2)$ et

$$
\varepsilon_{T_{0}}\left(v_{n}, \Psi_{n}\right) \leqslant \varepsilon^{*}, \quad\left\|v_{n}, \Psi_{n}\right\|_{N, \lambda, T_{0}} \leqslant 1 .
$$

Preuve. Par récurrence sur $n$. Supposons que l'on ait (7.2.3). On a

$$
f_{n}=f_{a}+\left(L\left(u_{a}, \phi_{a}\right)-L\left(u_{n}, \phi_{n}\right)\right) u_{a}
$$

$\left(u_{a}, \phi_{a}\right) \in C^{\infty}\left(\bar{\Omega}_{T_{0}}\right) \times C^{\infty}\left(\bar{\omega}_{T_{0}}\right)$, et a son support dans $\bar{\Omega}_{T_{0, \rho}} \times \bar{\omega}_{T_{0, \rho}}$. Avec (7.2.3), on peut appliquer la Proposition 5.4.2 et conclure que

$$
\left\|A\left(u_{n}, \phi_{n}\right)-A\left(u_{a}, \phi_{a}\right)\right\|_{N, \lambda-1 / 2, T^{\prime}} \leqslant C\left\{\left\|v_{n}\right\|_{N, \lambda-1 / 2, T^{\prime}}+\left\|j \Psi_{n}\right\|_{N, \lambda-1 / 2, T^{\prime}}\right\}
$$

avec $C$ indépendant de $\lambda, T^{\prime}$ et $n$. On tire alors du Lemme 7.1.3 l'estimation

$$
\left\|f_{n}\right\|_{N, \lambda-1 / 2, T^{\prime}} \leqslant C\left\{\left\|f_{a}\right\|_{N, \lambda-1 / 2, T^{\prime}}+\left\|v_{n}, \Psi_{n}\right\|_{N, \lambda-1, T^{\prime}}\right\} .
$$

Puisque $f_{a} \in H_{\lambda+1 / 2}^{N}\left(\Omega_{T_{0}}\right)$ et que l'on a supposé (7.2.3), on obtient l'estimation

$$
\left\|f_{n}\right\|_{N, \lambda-1 / 2, T} \leqslant C_{1} T^{\prime}
$$

avec $C_{1}$ indépendant de $T^{\prime}$ et $n$.

Par ailleurs on a

$$
\dot{g}_{n+1}=g_{a}-\left\{\mathscr{F}\left(\gamma u_{n}, \phi_{n}\right)-\mathscr{F}\left(\gamma u_{a}, \phi_{a}\right)-F_{\gamma u_{n}, \phi_{n}}\left(\gamma v_{n}, \Psi_{n}\right)\right\},
$$

et la Proposition 5.4.2 permet d'en déduire que

$$
\left\|g_{n+1}-g_{a}\right\|_{N, 2 \lambda-1, T^{\prime}} \leqslant C\left\{\left\|\gamma v_{n}\right\|_{N, \lambda, T^{\prime}}^{2}+\left\|\Psi_{n}\right\|_{N+1, \lambda+1, T^{\prime}}^{2}\right\} .
$$

Puisque $2 \lambda-1 \geqslant \lambda+1$ et que $g_{a} \in H_{\lambda+1}^{N}\left(\omega_{T_{0}}\right)$, on en tire, avec (7.2.3), que

$$
\left\|g_{n+1}\right\|_{N, \lambda, T} \leqslant C_{2} T^{\prime} \text {. }
$$

La Proposition 7.1.5 permet, grâce à (7.2.3), de résoudre le problème (7.2.2), et on trouve une solution $\left(w_{n+1}, \theta_{n+1}\right)$ qui, d'après (7.2.6) et (7.2.9) vérifie pour tout $T^{\prime} \leqslant T_{0}$,

$$
\left\|w_{n+1}, \theta_{n+1}\right\|_{N, \lambda, T^{\prime}} \leqslant C_{3} T^{\prime} .
$$

La fonction $\left(v_{n+1}, \phi_{n+1}\right)$ vérifie alors

$$
\varepsilon_{T_{0}}\left(v_{n+1}, \Psi_{n+1}\right) \leqslant C_{4}\left\|v_{n+1}, \Psi_{n+1}\right\|_{N, \lambda, T_{0}} \leqslant C_{3} C_{4} H_{1} T,
$$

et (7.2.3) à l'ordre $n+1$ en résulte pourvu que $T \leqslant T^{*}=\operatorname{Inf}\left(1 / C_{3} H_{1}, \varepsilon^{*} / C_{4} C_{3} H_{1}\right)$.

Nous étudions maintenant la convergence du schéma (7.2.1), (7.2.2). Celle-ci résulutera de la proposition suivante

Proposition 7.2.2. Il existe une constante $C$, telle que pour tout $T \leqslant T^{*}$, la suite $\left(u_{n}, \phi_{n}\right)$ définie par (7.2.1), (7.2.2) vérifie

$$
\left\|u_{n+2}-u_{n+1}, \phi_{n+2}-\phi_{n+1}\right\|_{N-1, \lambda, T} \leqslant C T \mid\left\|u_{n+1}-u_{n}, \phi_{n+1}-\phi_{n}\right\|_{N-1, \lambda, T} .
$$

Preuve. Posons $a_{n}=u_{n+1}-u_{n}, \alpha_{n}=\phi_{n+1}-\phi_{n}$ et

$$
b_{n}=L\left(u_{n+1}, \phi_{n+1}\right) a_{n+1}, \quad \beta_{n}=F_{\gamma u_{n+1}, \phi_{n+1}}\left(\gamma a_{n+1}, \alpha_{n+1}\right) \text {. }
$$


Puisque $v_{n}=w_{n}$ sur $\Omega_{T}$ et que $\Psi_{n}=\theta_{n}$ sur $\omega_{T}$, on a

$$
\begin{aligned}
& b_{n}=\left(L\left(u_{n}, \phi_{n}\right)-L\left(u_{n+1}, \phi_{n+1}\right)\right) u_{n+1} \quad \operatorname{sur} \Omega_{T}, \\
& \beta_{n}=-\mathscr{F}\left(\gamma u_{n+1}, \phi_{n+1}\right)+\mathscr{F}\left(\gamma u_{n}, \phi_{n}\right)+F_{\gamma u_{n}, \phi_{n}}\left(\gamma a_{n}, \alpha_{n}\right) \operatorname{sur} \omega_{T} .
\end{aligned}
$$

D'après la Proposition 7.2.1, la suite $\left(u_{n}, \phi_{n}\right)$ est borné dans $W_{T_{0}}$. On déduit de la Proposition 5.4.2 et du Lemme 7.1.3 que l'on a

$$
\left\|A\left(u_{n}, \phi_{n}\right)-A\left(u_{n+1}, \phi_{n+1}\right)\right\|_{N-1, \lambda-1 / 2, T} \leqslant C\left\|a_{n}, \alpha_{n}\right\|_{N-1, \lambda-1, T} .
$$

La Proposition 7.2.1 implique aussi que $\left\|\nabla u_{n}\right\|_{H^{n-1}\left(\Omega_{T_{0}}\right)}$, est borné, et la Proposition 5.3.1 conduit à la majoration

$$
\left\|b_{n}\right\|_{N-1, \lambda-1 / 2, T} \leqslant C\left\|a_{n}, \alpha_{n}\right\|_{N-1, \lambda-1, T} \leqslant C T\left\|a_{n}, \alpha_{n}\right\|_{N-1, \lambda, T}
$$

De même la Proposition 5.4.2 implique que

$$
\begin{aligned}
\left\|\beta_{n}\right\|_{N-1,2 \lambda-1, T} & \leqslant C\left\{\left\|\gamma a_{n}\right\|_{N-1, \lambda, T}^{2}+\left\|\alpha_{n}\right\|_{N, \lambda+1, T}^{2}\right\} \\
& \leqslant C\left\|a_{n}, \alpha_{n}\right\|_{N-1, \lambda, T}^{2} .
\end{aligned}
$$

Puisque $2 \lambda-1 \geqslant \lambda+1$, et que la suite $\left(a_{n}, \alpha_{n}\right)$ est bornée dans $W_{\lambda, T_{0}}^{N-1}$, on en déduit la majoration

$$
\left\|\beta_{n}\right\|_{N-1, \lambda, T} \leqslant C T\|\| a_{n}, \alpha_{n} \|_{N-1, \lambda, T}
$$

La Proposition 7.2.1 permet d'appliquer la Proposition 7.1.5 au système (7.2.11). On a donc

$$
\left\|a_{n+1}, \alpha_{n+1}\right\|_{N-1, \lambda, T} \leqslant C_{0}\left\|b_{n}, \beta_{n}\right\|_{N-1, \lambda, T}^{\prime}
$$

ce qui, compte tenu de (7.2.13), (7.2.14), achève la démonstration de la Proposition 7.2.2.

Démonstration $d u$ Théorème 3.3.1. La constante $C$ étant celle déterminée à la Proposition 7.2.2, on choisit $T \leqslant \operatorname{Inf}\left\{T^{*}, 1 / 2 C\right\}$. Le schéma itératif (7.2.1) (7.2.2) fournit une suite $\left(u_{n}, \phi_{n}\right)=\left(u_{a}, \phi_{a}\right)+\left(v_{n}, \Psi_{n}\right)$ bornée dans $W_{T_{0}}$, avec $\left(v_{n}, \Psi_{n}\right)$ de Cauchy dans $W_{\lambda, T}^{N-1}$. La limite $(u, \phi)=\left(u_{a}, \phi_{a}\right)+(v, \Psi)$ est donc dans l'espace $W_{T} \subset C^{2}\left(\bar{\Omega}_{T}\right) \times C^{3}\left(\bar{\omega}_{T}\right)$, et la convergence a lieu dans $C^{1}\left(\bar{\Omega}_{T}\right) \times C^{2}\left(\bar{\omega}_{T}\right)$. Passant à la limite dans (7.2.2) tenant compte du fait que $w_{n}=v_{n}$ sur $\Omega_{T}$ et que $\theta_{n}=\Psi_{n}$ sur $\omega_{T}$, on voit que

$$
\begin{array}{ll}
L(u, \phi) v=-L(u, \phi) u_{a} & \operatorname{sur} \Omega_{T}, \\
\mathscr{F}(\gamma u, \phi)=0 & \operatorname{sur} \omega_{T} .
\end{array}
$$

$(u, \phi)$ est donc solution du problème (7.1.5) sur $\Omega_{T} \times \omega_{T}$, et solution du problème initial (3.3.3) $\operatorname{sur} \Omega_{T, \rho / 2} \times \omega_{T, \rho / 2}$.

Appendice A-stabilité des chocs faibles. Le but de cet appendice est de vérifier, que pour un système strictement hyperbolique vraiment non linéaire les chocs faibles sont uniformément stables.

A.1. Condition de Lopatinski uniforme pour un système $2 \times 2$. Selon A. Majda [7], la stabilité des chocs plans conduit à expliciter la condition de Lopatinski pour un 
système de la forme

$$
\left\{\begin{array}{l}
L u=\frac{\partial u}{\partial t}+A \frac{\partial u}{\partial x}+B \frac{\partial u}{\partial y}=f \quad \text { pour } x>0 \\
F(\gamma u, \phi)=l \frac{\partial \phi}{\partial t}+p \frac{\partial \phi}{\partial y}+A u_{\mid x=0}=g
\end{array}\right.
$$

où les matrices réelles $A$ et $B$ sont constantes, ainsi que les vecteurs $l$ et $p$.

$L$ est supposé strictement hyperbolique dans la direction $\partial_{t}$ et $A$ est supposée avoir deux valeurs propres réelles $\lambda$ et $\mu$ telles que

$$
\lambda<0<\mu \text {. }
$$

Sans nuire à la généralité, nous supposerons $A$ diagonale; nous noterons alors $\left[\begin{array}{ll}a & c \\ b & d\end{array}\right]$ la matrice $B$, l'hypothèse d'hyperbolicité stricte se traduisant simplement par la condition

$$
b c>0
$$

Pour $\tau \in \mathbf{C}$ vérifiant $\operatorname{Im} \tau<0$ et pour $\eta \in \mathbf{R}$, avec $|\tau|+|\eta| \neq 0$ l'équation en $\xi$, $\operatorname{det}(\tau \mathrm{Id}+\xi A+\eta B)=0$ possède une unique racine $\xi_{+}(\tau, \eta)$ dans le demi-plan $\operatorname{Im} \xi>0$, et l'espace propre $E_{+}(\tau, \eta)=\operatorname{Ker}\left(\tau \operatorname{Id}+\xi_{+} A+\eta B\right)$ est de dimension 1 . En outre, $\xi_{+}$et $E_{+}$ont des limites lorsque $\operatorname{Im} \tau \rightarrow 0$, pour $|\tau|+\partial \eta \mid \neq 0$. Alors, la condition de Lopatinski uniforme pour le système (A.1.1) s'écrit

$$
\begin{aligned}
& \text { Pour tout } \tau \in \mathbf{C}, \operatorname{Im} \tau \leqslant 0 \text { et tout } \eta \in \mathbf{R} \text { avec }|\tau|+|\eta| \neq 0, \\
& \tau l+\eta p \notin A E_{+}(\tau, \eta) .
\end{aligned}
$$

Notons

$$
\left(\begin{array}{l}
l_{1} \\
l_{2}
\end{array}\right) \text { et }\left(\begin{array}{l}
p_{1} \\
p_{2}
\end{array}\right)
$$

les composantes de $l$ et $p$ (dans la base de diagonalisation de $A$ ). On introduit les quantités suivantes

$$
\left\{\begin{array}{l}
T_{0}=\frac{2 \sqrt{b c \mu|\lambda|}}{\mu-\lambda} ; \quad S_{0}=\sqrt{\frac{b c}{\mu|\lambda|}}, \\
\sigma=\frac{c l_{2}}{\mu l_{1}} ; \quad \sigma=\frac{c}{\mu} \frac{l_{1} p_{2}-p_{1} l_{2}}{l_{1}^{2}} ; \quad \theta=-\frac{p_{1}}{l_{1}}-\frac{\lambda d-\mu a}{\mu-\lambda} .
\end{array}\right.
$$

LEMME A.1. Sous les hypothèses suivantes:

(i) $l_{1} \neq 0$,

(ii) $b\left(l_{1} p_{2}-p_{1} l_{2}\right)>0$,

(iii) $|\theta|<T_{0}$,

(iv) $S_{0}>\left(\sigma+\rho /\left(T_{0}-\theta\right)\right)$ et $S_{0}>\left(\rho /\left(T_{0}+\theta\right)-\sigma\right)$,

le système (A.1.1) vérifie la condition de Lopatinski uniforme $(\mathrm{L})$.

REMARQue. La condition (i) est nécessaire, et si (i) et (ii) sont satisfaits, (iii) et (iv) deviennent nécessaires et suffisants pour la condition (L). 
Preuve. (1) pour $\eta=0$ on a $\xi_{+}=-\tau / \mu, E_{+}=\mathbf{C}\left(\begin{array}{l}0 \\ 1\end{array}\right)$ et $\tau l \notin A E_{+}$par la condition (i).

(2) On a $\xi_{+}(-\bar{\tau},-\eta)=-\overline{\xi_{+}(\tau, \eta)}, E_{+}(-\bar{\tau}, \eta)=\overline{E_{+}(\tau, \eta)}$. Par homogénéité, il nous suffit donc de montrer que $\forall \tau \in \mathbf{C}, \operatorname{Im} \tau \leqslant 0, \tau l+p \notin A E_{+}(\tau, 1)$.

Cela revient à dire que l'équation

$$
\xi_{+}(\tau, 1)(\tau l+p)+(\tau \operatorname{Id}+B) A^{-1}(\tau l+p)=0
$$

n'admet pas de solution $\tau$ avec $\operatorname{Im} \tau \leqslant 0$.

L'équation (A.1.5) implique que

$$
\xi_{+}\left(\tau l_{1}+p_{1}\right)+\left(\frac{\tau+a}{\lambda}\right)\left(\tau l_{1}+p_{1}\right)+\frac{c}{\mu}\left(\tau l_{2}+p_{2}\right)=0 .
$$

Donc si $\tau$ est solution de (A.1.5), on a d'abord $\left(\tau l_{1}+p_{1}\right) \neq 0$ par la condition (ii), et ensuite

$$
-\xi_{+}(\tau, 1)=\frac{\tau+a}{\lambda}+\frac{c}{\mu} \frac{l_{2}}{l_{1}}+\frac{c}{\mu} \frac{l_{1} p_{2}-p_{1} l_{2}}{l_{1}^{2}} \frac{1}{\tau+p_{1} / l_{1}} .
$$

Notons $f(\tau)$ le membre de droite de (A.1.6). La condition (ii), (A.1.2) et (A.1.3) impliquent que $\operatorname{Im} f(\tau)>0$ pour $\operatorname{Im} \tau<0$; par conséquent l'équation (A.1.5) n'a pas de solution pour $\operatorname{Im} \tau<0$.

(3) On sait déjà que si $\tau$ réel est solution de (A.1.5) alors $\tau \neq p_{1} / l_{1}$ et $\xi_{+}(\tau, 1)=$ $-f(\tau)$ est réel.

On simplifie les calculs en posant

$$
\tau=T+\frac{\lambda d-\mu a}{\mu-\lambda}, \quad \xi=X+\frac{a-d}{\mu-\lambda} .
$$

L'équation $\operatorname{det}(\tau+\xi A+\eta B)=0$ s'écrit, pour $\eta=1,(\lambda X+T)(\mu X+T)=b c$, et la fonction $X_{+}(T)$, correspondant à $\xi_{+}(\tau, 1)$, est non réelle pour $|T|<T_{0}$, réelle pour $|T| \geqslant T_{0}$, décroissante sur chaque intervalle ] $-\infty,-T_{0}$ ] et $] T_{0},+\infty[$, et asymptote à la droite $\mu X+T=0$. Après le changement (A.1.7), (A.1.6) devient, avec les notations (A.1.4).

$$
X_{+}(T)=-F(T)
$$

où $F(T)=T / \lambda+\sigma+\rho /(T-\theta)$.

On a déjà dit que (A.1.8) n'a pas de solution $T$ dans l'intervalle $]-T_{0}, T_{0}$ [; la condition (iii) assure que $F$ est définie et strictement décroisante sur ] $-\infty, T_{0}$ ] et $\left[T_{0}, \infty[\right.$; la condition (iv) traduit que

$$
\begin{aligned}
X_{+}\left(T_{0}\right) & =-\frac{\lambda+\mu}{2 \lambda \mu} T_{0}<-F\left(T_{0}\right), \\
X_{+}\left(-T_{0}\right) & =\frac{\lambda+\mu}{2 \lambda \mu} T_{0}>-F\left(-T_{0}\right),
\end{aligned}
$$

et par conséquent (A.1.8) n'a pas non plus de solution sur $|T| \geqslant T_{0}$.

A.2. Chocs faibles. Considérons un système de deux lois de conservation

$$
\frac{\partial u}{\partial t}+\frac{\partial}{\partial x} f(u)+\frac{\partial}{\partial y} g(u)=0
$$


et un choc plan

$$
u= \begin{cases}u_{-} & \text {pour } x<\sigma t \\ u_{+} & \text {pour } x>\sigma t\end{cases}
$$

On a donc

$$
\sigma\left(u_{+}-u_{-}\right)=f\left(u_{+}\right)-f\left(u_{-}\right) .
$$

Notons $A^{+}, B^{+}$[resp. $A^{-}, B^{-}$], les matrrices $f^{\prime}\left(u_{+}\right), g^{\prime}\left(u_{+}\right)$[resp. $f^{\prime}\left(u_{-}\right)$, $\left.g^{\prime}\left(u_{-}\right)\right]$. On supose que les opérateurs $L_{ \pm}=\partial / \partial t+A^{ \pm}(\partial / \partial x)+B^{ \pm}(\partial / \partial y)$ sont strictement hyperboliques dans la direction $\partial_{t}$. Enfin on note $\lambda_{1}^{ \pm}<\lambda_{2}^{ \pm}$les valeurs propres de $A^{ \pm}$.

Suivant Lax [5] on dit que (A.2.2) est un 1-choc si l'on a

$$
\lambda_{1}^{+}<\sigma<\lambda_{1}^{-} \text {et } \sigma<\lambda_{2}^{+}
$$

et que c'est un 2-choc si

$$
\lambda_{2}^{+}<\sigma<\lambda_{2}^{-} \text {et } \sigma>\lambda_{1}^{-} \text {. }
$$

Selon A. Majda [7], le 1-choc [resp. 2-choc] est uniformément stable, si le problème suivant satisfait à la condition de Lopatinski uniforme:

$$
\left\{\begin{array}{l}
\frac{\partial v}{\partial t}+\left(A^{+}-\sigma\right) \frac{\partial v}{\partial x}++B^{+} \frac{\partial v}{\partial y}=f \quad \text { pour } x>0 \\
\left(u_{+}-u_{-}\right) \frac{\partial \phi}{\partial t}+\left(g\left(u_{+}\right)-g\left(u_{-}\right)\right) \frac{\partial \phi}{\partial y}\left(A^{+}-\sigma\right) v_{\mid x=0}=g
\end{array}\right.
$$

[resp.

$$
\left\{\begin{array}{ll}
\frac{\partial v}{\partial t}+\left(\sigma-A^{-}\right) \frac{\partial v}{\partial x}+B^{-} \frac{\partial v}{\partial y}=f & \text { pour } x>0 \\
\left(u_{+}-u_{-}\right) \frac{\partial \phi}{\partial t}+\left(g\left(u_{+}\right)-g\left(u_{-}\right)\right) \frac{\partial \phi}{\partial y}+\left(A^{-}-\sigma\right) v_{\mid x=0}=g
\end{array}\right] .
$$

Donnons-nous maintenant $u_{0} \in \mathbf{R}^{2}$; supposons que $L_{0}=\partial / \partial t+f^{\prime}\left(u_{0}\right)(\partial / \partial x)+$ $g^{\prime}\left(u_{0}\right)(\partial / \partial y)$ est strictement hyperbolique dans la direction $\partial_{t}$. Supposons aussi que $f^{\prime}(u)$ est vraiment non linéaire, au voisinage de $u_{0}$, c'est à dire que

$$
r_{i} \cdot \text { grand }_{u} \lambda_{i} \neq 0 \text { pour } i=1,2
$$

en désignant par $\lambda_{1}(u)<\lambda_{2}(u)$ et $r_{1}(u), r_{2}(u)$ les valeurs propres et vecteurs propres de $f^{\prime}(u)$.

Lemme A.2. Sous les hypothèses ci-dessus, il existe un voisinage $V$ de $u_{0}$ tel que tous les 1-chocs et tous les 2-chocs (A.2.2) avec $\left(u_{+}, u_{-}\right) \in V \times V$ sont uniformément stables.

Preuve. Faisons la démonstration pour les 1-chocs. Selon Lax [5], sur un voisinage $v$ de $u_{0}$, les couples $\left(u_{-}, u_{+}\right)$formant un 1-choc sont ceux de la forme $u_{+}=\mathscr{F}\left(\varepsilon, u_{-}\right)$avec $0<\varepsilon \leqslant \varepsilon_{0}$, où $\mathscr{F}$ est une fonction $C^{\infty}$; après normalisation on peut supposer que

$$
u_{+}-u_{-}=\varepsilon r_{1}\left(u_{+}\right)+O\left(\varepsilon^{2}\right), \quad \lambda_{1}^{+}-\sigma=-\varepsilon .
$$


On a alors

$$
g\left(u_{+}\right)-g\left(u_{-}\right)=\varepsilon B^{+} r_{1}\left(u_{+}\right)+O\left(\varepsilon^{2}\right)
$$

Notant $\left[\begin{array}{ll}a & c \\ b & d\end{array}\right]$ la matrice de $B^{+}$dans la base $r_{1}\left(u_{+}\right), r_{2}\left(u_{+}\right)$, on évalue grâce à (A.2.8) et (A.2.9) les quantités introduites en (A.1.4)

$$
\begin{gathered}
T_{0}=\frac{2 \sqrt{b c\left(\lambda_{2}^{+}-\sigma\right)}}{\lambda_{2}^{+}-\lambda_{1}^{+}} \sqrt{\varepsilon} ; \quad S_{0}=\frac{\sqrt{b c}}{\sqrt{\lambda_{2}^{+}-\sigma}} \frac{1}{\sqrt{\varepsilon}} \\
\sigma=O(\varepsilon) ; \quad \rho=\frac{b c}{\lambda_{2}^{+}-\sigma}+O(\varepsilon) ; \quad \theta=O(\varepsilon),
\end{gathered}
$$

Puisque $b c$ et $\lambda_{2}^{+}-\lambda_{1}^{+}$restent minorés par un nombre strictement positif pour $u_{+}$dans un voisinage de $u_{0}$, un simple calcul montre que les hypothèses du Lemme A.1 sont vérifiées pour $\varepsilon$ assez petit.

A. 3. Verification des Remarques 2.1.1 et 2.2.3. Il nous reste à vérifier quel'hypothèse $\left(\mathrm{H}_{3}\right)$ est satisfaite pour les chocs faibles, pour un système vraiment non linéaire. Dans la situation (2.1.8) de deux chocs vérifiant (2.1.9) on a, pour des chocs faibles

$$
\begin{gathered}
\underline{u}^{\#-} \underline{u}^{-}=\varepsilon r_{1}\left(\underline{u}^{\#}\right)+O\left(\varepsilon^{2}\right), \quad \underline{u}^{+}-\underline{u}^{\#}=\eta r_{2}\left(\underline{u}^{\#}\right)+O\left(\eta^{2}\right), \\
\lambda_{1}\left(u^{\#}\right)-\rho=-\varepsilon<0 ; \quad \lambda_{2}\left(u^{\#}\right)-\sigma=\eta>0 .
\end{gathered}
$$

Avec les notations de $\left(\mathrm{H}_{3}\right)$ on a donc $\alpha=\delta=1$ et $\beta=0(\varepsilon), \gamma=0(\eta)$, si bien que la condition $|\gamma \beta|<|\alpha \delta|$ est trivialement vérifiée pour $\varepsilon$ et $\eta$ assez petits.

Appendice B. Problème mixte à coefficients $C^{2}$. Nous indiquons ici une démonstration du Théorème 6.2.1, dont l'idée principale est d'utiliser les méthodes paradifférentielles de J. M. Bony [2] pour obtenir les inégalites d'énergie $L^{2}$, pour un problème à coefficients $C^{2}$. On améliore ainsi très légèrement le Theoréme 2 de $\mathrm{A}$. Majda [7].

B.1. Calcul paradifférentiel à paramétre. Nous reprenons d'abord les principales définitions et les principaux résultats de J. M. Bony [2] et Y. Meyer [9, 10] dans le cas "à paramètre".

Notons $x$ la variable de $\mathbf{R}^{n}$ et $\xi$ la variable duale. Pour $\lambda \geqslant 1$ on note $\Lambda=$ $\left(\lambda^{2}+\left|D_{x}\right|^{2}\right)^{1 / 2}$, et on munit $H^{s}$ des normes

$$
\|u\|_{s, \lambda}=\left\|\Lambda^{s} u\right\|_{L^{2}}
$$

Soit $\chi \in \mathrm{C}^{\infty}([0, \infty[)$ à support dans $[0,2]$, valant 1 sur $[0,1]$. Pour $j \geqslant 0$ on pose

$$
S_{j}\left(D_{x}\right)=\chi\left(2^{-j}\left|D_{x}\right|\right), \quad \Delta_{j, \lambda}\left(D_{x}\right)=\chi\left(2^{-j-1} \Lambda\right)-\chi\left(2^{-j} \Lambda\right)
$$

Pour $\rho>0$ entier, $C^{\rho}$ désigne l'espace des fonctions de classe $C^{\rho}$ sur $\mathbf{R}^{n}$, bornées ainsi que leurs dérivées. Pour $\rho$ non entier, $C^{\rho}$ est l'espace de Hölder (uniforme) usuel sur $\mathbf{R}^{n}$. Pour $m$ réel et $\rho>0, \Gamma_{\rho}^{m}$ désigne l'ensemble des symboles $a(x, \xi, \lambda)$, de classe $C^{\rho}$ en $x$ et $C^{\infty}$ en $\xi$, et tels que, pour tout $\alpha \in \mathbf{N}^{n}$ il existe $C_{\alpha}$ pour lequel

$$
\forall \xi \in \mathbf{R}^{n}, \forall \lambda \geqslant 1 \quad\left\|\partial_{\xi}^{\alpha} a(\cdot, \xi, \lambda)\right\|_{C^{\rho}} \leqslant C_{\alpha}(\lambda+|\xi|)^{m-|\alpha|} .
$$

On désigne par $\Sigma_{\rho}^{m}$ l'ensemble des symboles $\sigma \in \Gamma_{\rho}^{m}$, dont la transformée de Fourier partielle en $x, \hat{\sigma}(\eta, \xi, \lambda)$ est supportée dans une région $|\eta| \leqslant \varepsilon(\lambda+|\xi|)$ pour un $\varepsilon<1$. 
On introduit la fonction

$$
G(y, \xi, \lambda)=(2 \Pi)^{-n} \int e^{i \cdot \eta \eta} \sum_{j \geqslant 2} S_{j-2}(\eta) \Delta_{j, \lambda}(\xi) d \eta
$$

qui vérifie

$$
\left\|\partial_{\xi}^{\alpha} G(\cdot, \xi, \lambda)\right\|_{L^{1}\left(\mathbf{R}^{n}\right)} \leqslant C_{\alpha}(\lambda+|\xi|)^{-|\alpha|} .
$$

A $a \in \Gamma_{\rho}^{m}$, on associe le symbole

$$
\sigma_{a}(x, \xi, \lambda)=\int G(x-y, \xi, \lambda) a(y, \xi, \lambda) d y
$$

et il résulte de (B.1.5) et des définitions (B.1.2) que $\sigma_{a} \in \sum_{\rho}^{m}$. On note alors $T_{a}^{\lambda}$ l'opérateur $\sigma_{a}\left(x, D_{x}, \lambda\right)$. En particulier, si a est une fonction $a(x) \in C^{\rho}\left(\mathbf{R}^{n}\right)$ on a:

$$
T_{a}^{\lambda} u=\sum_{j \geqslant 2} S_{j-2}\left(D_{x}\right) a \Delta_{j, \lambda}\left(D_{x}\right) u
$$

Convenons de dire qu'une famille $R_{\lambda}$ d'opérateurs est bornée de $H^{s}$ dans $H^{s^{\prime}}$ uniformément en $\lambda$, s'il existe une constante $C$ telle que pour tout $u \in H^{s}$ et tout $\lambda \geqslant 1$ on ait $R_{\lambda} u \in H^{s^{\prime}}$ avec $\left\|R_{\lambda} u\right\|_{s^{\prime}, \lambda} \leqslant C\|u\|_{s, \lambda}$.

On peut alors énconcer

Proposition B.1.1. Soit $\sigma \in \Sigma_{\rho}^{m}$; alors pour tout $s \in \mathbf{R}, \sigma\left(x, D_{x}, \lambda\right)$ est borné de $H^{s}$ dans $H^{s-m}$, uniformément en $\lambda$.

Ce résultat s'applique en particulier aux symboles $\sigma_{a}$ (B.1.6) et on a

Proposition B.1.2. Soit $a \in \Gamma_{\rho}^{m}$; alors $T_{a}^{\lambda}$ est borné de $H^{s}$ dans $H^{s-m}$ uniformément en $\lambda$; en outre si $\rho>m \geqslant 0, T_{a}^{\lambda}-a\left(x, D_{x}, \lambda\right)$ est borné de $L^{2}$ dans $L^{2}$, uniformément en $\lambda$.

En outre, on a le calcul symbolique suivant:

Proposition B.1.3. (i) Soit $a \in \Gamma_{\rho}^{m}$ avec $\rho>1$. Alors $\left(T_{a}^{\lambda}\right)^{*}-T_{\bar{a}}^{\lambda}$ est borné de $H^{s}$ dans $H^{s-m+1}$, uniformément en $\lambda$.

(ii) Soit $a \in \Gamma_{\rho}^{m}, b \in \Gamma_{\rho}^{m^{\prime}}$ avec $\rho>1$. Alors $T_{a}^{\lambda} \circ T_{b}^{\lambda}-T_{a b}^{\lambda}$ est borné de $H^{s}$ dans $H^{s-m-m^{\prime}+1}$ uniformément en $\lambda$.

Enfin, on a les inégalités de Garding suivantes:

Proposition B.1.4. (i) Soit $a \in \Gamma_{\rho}^{m}$ avec $\rho>1$, tel que

$$
\operatorname{Re} a(x, \xi, \lambda) \geqslant(\lambda+|\xi|)^{m} \text {. }
$$

Alors existe $C$ et $c$ tels que pour tout $\lambda \geqslant 1$ et tout $u \in H^{m}$, on ait

$$
C\|u\|_{m / 2, \lambda}^{2} \leqslant \operatorname{Re}\left(T_{a}^{\lambda} u, u\right)+C\|u\|_{(m-1) / 2, \lambda}^{2} .
$$

(ii) Soit $a \in \Gamma_{\rho}^{m}$ avec $\rho \geqslant 2$, tel que $\operatorname{Re} a(x, \xi, \lambda) \geqslant 0$. Alors il existe $C$ tel que pour tout $\lambda \geqslant 1$ et tout $u \in H^{m}$, on ait

$$
-C\|u\|_{(m-1) / 2, \lambda}^{2} \leqslant \operatorname{Re}\left(T_{a}^{\lambda} u, u\right) .
$$


Pour la preuve de ces propositions, on renvoie à J. M. Bony [2] et Y. Meyer [9, 10], l'introduction du paramètre $\lambda$, suivant les régles indiquées en (B.1.2), (B.1.6), ne modifiant en rien les démonstrations. Nous allons cependant reprendre la démonstration du point (ii) de la Proposition B.1.4, pour bien mettre en évidence le rôle de l'hypothèse $\rho \geqslant 2$.

On se ramène d'abord au cas $m=1$. Soit alors $\zeta$ une fonction $C^{\infty}$, réelle et paire, telle que $\hat{\zeta}$ soit à support compact, et vérifiant: $\|\zeta\|_{L^{2}\left(\mathbf{R}^{n}\right)}=1$. Soit $W$ l'opérateur de $L^{2}\left(\mathbf{R}^{n}\right)$ dans $L^{2}\left(\mathbf{R}^{2 n}\right)$,

$$
W u(x, \xi)=\phi(\varepsilon)^{n / 2} \int e^{i x-y \cdot \xi} \zeta(\phi(\xi)(x-y)) u(y) d y
$$

où $\phi(\xi)=\left(\lambda^{2}+|\xi|^{2}\right)^{1 / 4}$. L'adjoint $W^{*}$ de $W$ est

$$
W^{*} F(x)=(2 \pi)^{n} \int e^{i x-y \cdot \xi} \zeta(\phi(\xi)(x-y)) F(y, \xi) \phi(\xi)^{n / 2} d y d \xi .
$$

L'opérateur $B_{\lambda}=W^{*} a W$ est positif par construction et on a,

$$
B_{\lambda} u(x)=(2 \pi)^{-n} \int e^{i x-y \cdot \xi} b(x, y, \xi, \lambda) u(y) d y d \xi
$$

avec

$$
b(x, y, \xi, \lambda)=\phi(\xi)^{n} \int \zeta(\phi(\xi)(x-z)) \zeta(\phi(\xi)(z-y)) a(z, \xi, \lambda) d z .
$$

On voit que

$$
\left|\partial_{x, y}^{\alpha} \partial_{\xi}^{\beta} b(x, y, \xi, \lambda)\right| \leqslant C_{\alpha, \beta}(\lambda+|\xi|)^{1-|\beta|+|\alpha| / 2}
$$

c'est à dire que $b$ est dans une classe de symboles $S_{1,1 / 2}^{1}$ associée à la fonction poids $\lambda+|\xi|$. On en conclut que

$$
B_{\lambda}=b\left(x, x, D_{x}, \lambda\right)+\sum_{j=1}^{n}\left(\partial_{\xi_{j}} D_{y_{j}} b\right)\left(x, x, D_{x}, \lambda\right)+R_{\lambda}
$$

où $R_{\lambda} \in S_{1,1 / 2}^{0}$ est borné dans $L^{2}$, uniformément en $\lambda$.

Posons $b(x, \xi, \lambda)=b(x, x, \xi, \lambda)$ et $\sigma(x, \xi, \lambda)=\sigma_{a}(x, \xi, \lambda)$. On a

$$
\hat{b}(\eta, \xi, \lambda)=\phi(\xi)^{-n}(\hat{\zeta} * \hat{\zeta})(\eta / \phi(\xi)) \hat{a}(\eta, \xi, \lambda) .
$$

Si le support de $\hat{\zeta}$ est assez petit, $\hat{b}$ a son support contenu dans $|\eta| \leqslant \frac{1}{4} \phi(\xi)$ $\leqslant \frac{1}{4}(\lambda+|\xi|)$, et sur le support de $\hat{b}, \hat{a}=\hat{\sigma}$. Compte tenu de la parité de $\zeta$ on en déduit que

$$
b(x, \xi, \lambda)=\sigma(x, \xi, \lambda)+\phi(\xi)^{n} \int \zeta^{2}(\phi(\xi)(x-z)) \tilde{\sigma}(x, z, \xi, \lambda) d z
$$

avec

$$
\tilde{\sigma}(x, z, \xi, \lambda)=\sigma(z, \xi, \lambda)-\sigma(x, \xi, \lambda)-\sum_{j=1}^{n}\left(z_{j}-x_{j}\right) \partial_{x_{j}} \sigma(x, \xi, \lambda) .
$$

Puisque $a \in \Gamma_{\rho}^{1}$, avec $\rho \geqslant 2$, on a $\sigma \in \Sigma_{\rho}^{1}$ et

$$
\tilde{\sigma}(x, z, \xi, \lambda) \leqslant C_{\alpha}|x-z|^{2}(\lambda+|\xi|)^{-1-|\alpha|} .
$$


Avec (B.1.10), il en résulte que

$$
\left|\partial_{\xi}^{\alpha}(b-\sigma)(x, \xi, \lambda)\right| \leqslant C_{\alpha}(\lambda+|\xi|)^{-|\alpha|}
$$

et par suite que $b-\sigma \in \Sigma_{0}^{0}$. Avec la Proposition B.1.1 on en déduit que $b\left(x, D_{x}, \lambda\right)$ $-T_{a}^{\lambda}$ est borné dans $L^{2}$ uniformément en $\lambda$.

Utilisant la parité de $\zeta$ et le même argument de support qu'en (B.1.9), on a aussi

$$
\begin{aligned}
& \left(D_{y_{j}} b\right)(x, x, \xi, \lambda) \\
& \quad=\phi(\xi)^{n+1} \int \frac{1}{2}\left(D_{z} \zeta\right)^{2}(\phi(\xi)(x-z))(\sigma(z, \xi, \lambda)-\sigma(x, \xi, \lambda)) d z
\end{aligned}
$$

d'où l'on tire que $\left(D_{y_{j}} b\right)(x, x, \xi, \lambda) \in \Sigma_{\rho-1}^{1}$ puisque $\left(\partial_{\xi_{j}} D_{y_{j}} b\right)(x, x, \xi, \lambda) \in \Sigma_{\rho-1}^{0}$.

Finalement, avec (B.1.8), on voit que $B_{\lambda}-T_{a}^{\lambda}$ est borné dans $L^{2}$, uniformément en $\lambda$; puisque $B$ est positif par construction, l'inégalité

$$
\operatorname{Re}\left(T_{a}^{\lambda} u, u\right) \geqslant-C\|u\|_{0}^{2}
$$

en résulte.

B.2. Inégalités d'énergie. Nous reprenons les notations du paragraphe 6.2 , en oubliant les tildas. $\Omega$ est l'ouvert $\mathbf{R} \times] 0,1[\times \mathbf{R}$, et $L$ est un opérateur de la forme (6.2.1), associé à des conditions aux limites, $F(\gamma u, \phi)$, du type (6.2.2), $(\underline{L}, \underline{F})$ est un système du même type, à coefficients constants, et vérifiant les hypothèses $(\alpha)$ et $(\beta)$.

Proposition B.2.1. Il existe $\varepsilon_{0}>0$ et des fonctions $\lambda_{0}(K)$ et $C_{0}(K)$ telles que si l'on $a$

$$
\varepsilon(L, F) \leqslant \varepsilon_{0}, \quad\|L, F\|_{C^{2}} \leqslant K
$$

alors pour tout $\lambda \geqslant \lambda_{0}(K)$, tout $(u, \phi) \in C_{0}^{\infty}(\mathbf{R} \times[0,1] \times \mathbf{R}) \times C_{0}^{\infty}\left(\mathbf{R}^{2}\right)$ on a

(B.2.2) $\lambda\|u\|_{L_{\lambda}^{2}}^{2}+\|\gamma u\|_{L_{\lambda}^{2}}^{2}+\|\phi\|_{H_{\lambda}^{1}}^{2} \leqslant C_{0}(K)\left\{\frac{1}{\lambda}\|L u\|_{L_{\lambda}^{2}}^{2}+\|F(\gamma u, \phi)\|_{L_{\lambda}^{2}}^{2}\right\}$

où $\|u\|_{L_{\lambda}^{2}}$ désigne la norme $\left\|e^{-\lambda t} u\right\|_{L^{2}}$ sur $\Omega$, comme sur $\mathbf{R}^{2}$, et

$$
\|\phi\|_{H_{\lambda}^{1}}=\left(\|\nabla \phi\|_{L_{\lambda}^{2}}^{2}+\|\phi\|_{L_{\lambda}^{2}}^{2}\right)^{1 / 2} \text {. }
$$

Preuve. Notons $e(t, z, \tau, \zeta, \lambda)=(i \tau+\lambda) l^{\prime}(t, z)+i \zeta p^{\prime}(t, z)$. Les vecteurs $\underline{l}^{\prime}$ et $\underline{p}^{\prime}$ sont indépendants, et donc $e^{\prime} \neq 0$ pour $|\tau|+\lambda+|\zeta| \neq 0$, si $\varepsilon_{0}$ est assez petit. Notons $\pi$ le projecteur sur $\mathbf{C} \cdot e$ :

$$
\pi(t, z, \tau, \zeta, \lambda) v=(v, e) /|e|^{2}
$$

Alors, on pose

$$
k(t, z, \tau, \zeta, \lambda)=(I-\pi(t, z, \tau, \zeta, \lambda)) m^{\prime}(t, z) .
$$

Enfin, on introduit

$$
r(t, \theta, z, \tau, \zeta, \lambda)=G^{-1}(t, \theta, z)\{(i \tau+\lambda) \operatorname{Id}+i \zeta C(t, \theta, z)\}
$$


L'hypothèse de stabilité uniforme pour le problème (6.2.3) implique l'existence d'un symétriseur de Kreiss [4], $s(t, \theta, z, \tau, \zeta, \lambda)$, défini pour $\theta$ assez petit de classe $C^{2}$ en $(t, \theta, z), C^{\infty}$ et homogène de degré 0 en $(\tau, \zeta, \lambda) \in \mathbf{R}^{3} / 0$ (cf. A. Majda [7]). Ce symétriseur vérifie

(i) $s=s^{*}$,

(ii) il existe $C_{0}>0$ tel que $s r+r^{*} s \geqslant \lambda C_{0}$ Id,

(iii) il existe $C_{1}$ et $C_{1}$ tels que, pour $\theta=0$, on ait $s+C_{1} k^{*} k \geqslant C_{1}$ Id.

Enfin on introduit les symboles $e_{1}, \pi_{1}, k_{1}, r_{1}$ et $s_{1}$ obtenus en substituant $\rho(t) \zeta$ à $\zeta$. Ils vérifient encore (B.2.5).

Notons ici $x=(t, z)$, et considérons $s_{1}$ comme un symbole de $\Gamma_{2}^{0}$ en $x$, dépendant de façon continue de $\theta \in\left[0, \theta_{0}\right]$. On a aussi $\partial s_{1} / \partial \theta \in \Gamma_{1}^{0}$.

L'opérateur $e^{-\lambda t} G^{-1} L e^{\lambda t}$ s'écrit

$$
\partial / \partial \theta+r_{1}\left(t, \theta, z, D_{t}, D_{z}, \lambda\right),
$$

et, avec la Proposition B.1.2 il s'écrit

$$
L_{\lambda}=\partial / \partial \theta+T_{r_{1}}^{\lambda}+R_{\lambda}
$$

avec $R_{\lambda}$ borné dans $L^{2}$, uniformément en $\lambda$.

Posons $S=\frac{1}{2}\left(T_{s_{1}}^{\lambda}+\left(T_{s_{1}}^{\lambda}\right)^{*}\right)$. Alors $[\partial / \partial \theta, S]=\frac{1}{2}\left(T_{\partial s_{1} / \partial \theta}+\left(T_{\partial s_{1} / \partial \theta}\right)^{*}\right)$, est borné dans $L^{2}$, uniformément en $\lambda$.

Pour $u \in C_{0}^{\infty}\left(\mathbf{R} \times\left[0, \theta_{0}[\times \mathbf{R})\right.\right.$, en intégrant $\operatorname{Re} S L_{\lambda} u \bar{u}$ sur $\Omega$, on obtient, grâce au calcul symbolique de la Proposition B.1.3 et à (B.2.5)

$$
\lambda\|u\|_{0}^{2}+\left\|\gamma^{\prime} u\right\|_{0}^{2} \leqslant C\left\{\left\|L_{\lambda} u\right\|_{0}\|u\|_{0}+\left\|T_{k}^{\lambda} \gamma^{\prime} u\right\|_{0}^{2}+\|u\|_{0}^{2}+\left\|\gamma^{\prime} u\right\|_{-1, \lambda}^{2}\right\}
$$

et on en tire, que pour $\lambda$ assez grand

$$
\lambda\|u\|_{0, \lambda}^{2}+\left\|\gamma^{\prime} u\right\|_{0, \lambda}^{2} \leqslant C\left\{\lambda^{-1}\left\|L_{\lambda} u\right\|_{0, \lambda}^{2}+\left\|T_{k}^{\lambda} \gamma u\right\|_{0, \lambda}^{2}\right\}
$$

On a aussi $e^{-\lambda t} F^{\prime}\left(\gamma^{\prime} e^{\lambda t} u, e^{\lambda t} \phi^{\prime}\right)=F_{\lambda}^{\prime}\left(\gamma^{\prime} u, \phi^{\prime}\right)$ avec

$$
F_{\lambda}^{\prime}\left(\gamma^{\prime} u, \phi^{\prime}\right)=T_{e_{1}}^{\lambda} \phi^{\prime}+T_{m^{\prime}}^{\lambda} \gamma^{\prime} u+O\left(\left\|\phi^{\prime}\right\|_{L^{2}}+\left\|\gamma^{\prime} u\right\|_{-1, \lambda}\right) .
$$

Puisque $\left(1-\pi_{1}\right) e_{1}=0$ on a

$$
T_{k_{1}}^{\lambda} \gamma^{\prime} u=T_{1-\pi_{1}}^{\lambda} F_{\lambda}^{\prime}\left(\gamma^{\prime} u, \phi^{\prime}\right)+O\left(\left\|\phi^{\prime}\right\|_{0}+\left\|\gamma^{\prime} u\right\|_{-1, \lambda}\right) .
$$

De même, puisque $e_{1}^{*} e_{1}$ est elliptique de degré 2 , on a

$$
\begin{aligned}
\|\phi\|_{1, \lambda} & \leqslant C\left(\left\|T_{e_{1}}^{\lambda} \phi^{\prime}\right\|_{0}+\left\|\phi^{\prime}\right\|_{0}\right) \\
& \leqslant C\left\{\left\|F_{\lambda}\left(\gamma^{\prime} u, \phi^{\prime}\right)\right\|_{0}+\left\|\gamma^{\prime} u\right\|_{0}+\left\|\phi^{\prime}\right\|_{0}\right\}
\end{aligned}
$$

On déduit de (B.2.9) (B.2.11) que, pour $\lambda$ assez grand

$$
\lambda\|u\|_{0}^{2}+\left\|\gamma^{\prime} u\right\|_{0}^{2} \leqslant C\left\{\lambda^{-1}\left\|L_{\lambda} u\right\|_{0}^{2}+\left\|F_{\lambda}\left(\gamma^{\prime} u, \phi^{\prime}\right)\right\|_{0}^{2}+\left\|\phi^{\prime}\right\|_{0}^{2}\right\},
$$


et avec (B.2.12) on obtient l'estimation (B.2.2) pour $u \in C_{0}^{\infty}\left(\mathbf{R} \times\left[0, \theta_{0}[\times \mathbf{R}), \phi^{\prime} \in\right.\right.$ $C_{0}^{\infty}\left(\mathbf{R}^{2}\right)$.

De même, l'hypothèse de stabilité pour le problème (6.2.4), conduit à l'estimation (B.2.2) pour $\left.\left.u \in C_{0}^{\infty}(\mathbf{R} \times] \theta_{1}, 1\right] \times \mathbf{R}\right)$ et $\phi^{\prime \prime} \in C_{0}^{\infty}\left(\mathbf{R}^{2}\right)$.

Enfin, en utilisant un symétriseur paradifférentiel en $(\theta, z)$ que l'on construit grâce l'hypothèse de stricte hyperbolicité de $L$, on établit (B.2.2) pour $u \in C_{0}^{\infty}(\Omega)$.

La Proposition B.2.1, s'en déduit par partition de l'unité en $\theta$.

B.3. Existence-regularité des solutions. Pour le problème

$$
L u=f, \quad F(\gamma u, \phi)=g .
$$

A. Majda [7] a montré l'existence d'une solution par une méthode de régularisation. Une méthode classique de dualité conduit aussi au résultat.

Supposons d'abord que le coefficient $q$ de (6.2.2) soit identiquement nul. Alors, $l^{\prime}$ et $p^{\prime}$ [resp. $l^{\prime \prime}$ et $p^{\prime \prime}$ ] étant linéairement indépendants (pour $\varepsilon_{0}$ assez petit), l'équation $F(\gamma u, \phi)=g$, peut se mettre sous la forme

$$
\nabla \phi^{\prime}+M^{\prime} \gamma^{\prime} u=h^{\prime}, \quad \nabla \phi^{\prime \prime}+M^{\prime \prime} \gamma^{\prime \prime} u=h^{\prime \prime},
$$

où $h$ s'exprime linéairement en fonction de $g$.

Ce système est un système surdéterminé en $\left(\phi^{\prime}, \phi^{\prime \prime}\right)$; les conditions de compatibilité s'écrivent

$$
\operatorname{rot}\left(M^{\prime} \gamma^{\prime} u-h^{\prime}\right)=0, \quad \operatorname{rot}\left(M^{\prime \prime} \gamma^{\prime \prime} u-h^{\prime \prime}\right)=0,
$$

si, pour $w=\left(w_{1}, w_{2}\right), \operatorname{rot} w=\partial w_{2} / \partial t-\partial w_{1} / \partial z$.

Posons $H^{\prime}(t, z)=G_{0}^{*}(t, 0, z), H^{\prime \prime}(t, z)=-G_{1}^{*}(t, 1, z), N^{\prime}=\left(M^{* *}\right)^{-1} H^{\prime}$ et $N^{\prime \prime}$ $=\left(M^{\prime \prime *}\right)^{-1} H^{\prime \prime}$. Formellement on

$$
(L u, v)_{L^{2}(\Omega)}-\left(u, L^{*} v\right)_{L^{2}(\Omega)}=-\left(\gamma^{\prime} u, H^{\prime} \gamma^{\prime} v\right)_{L^{2}\left(\mathbf{R}^{2}\right)}-\left(\gamma^{\prime \prime} u, H^{\prime \prime} \gamma^{\prime \prime} v\right)_{L^{2}\left(\mathbf{R}^{2}\right)}
$$

et

$$
(L u, v)_{L^{2}(\Omega)}+(M \gamma u, N \gamma v)_{L^{2}\left(\mathbf{R}^{n}\right)}=\left(u, L^{*} v\right)_{L^{2}(\Omega)} .
$$

Si l'on note $\operatorname{div}\left(w_{1}, w_{2}\right)=\partial w_{1} / \partial t+\partial w_{2} / \partial z$, le problème $\left(L^{*}, \operatorname{div} N_{\gamma}\right)$ est adjoint au problème $\left(L, \operatorname{rot} M_{\gamma}\right)$, et vérifie donc la condition de Lopatinski uniforme rétrograde (le caractère différentiel ou pseudodifférentiel des conditions aux limites, n'intervient pas dans la formulation algébrique de cette condition). On en déduit, que pour $\lambda$ assez grand on a

$$
\lambda\|v\|_{L_{-\lambda}^{2}}^{2}+\|\gamma v\|_{L_{-\lambda}^{2}}^{2} \leqslant \frac{C}{\lambda}\left\|L^{*} v\right\|_{L_{-\lambda}^{2}}^{2}
$$

pour tout $v$ dans l'espace $\mathscr{E}$ des fonctions de $H_{-\lambda}^{1}$ telles que $\operatorname{div} N \gamma v=0$.

Pour $(f, h) \in L_{\lambda}^{2}(\Omega) \times L_{\lambda}^{2}\left(\mathbf{R}^{2}\right)$, on en déduit l'existence d'un $u \in L_{\lambda}^{2}(\Omega)$ tel que

$$
(f, v)_{L^{2}(\Omega)}+(h, N \gamma v)_{L^{2}\left(\mathbf{R}^{2}\right)}=\left(u, L^{*} v\right)_{L^{2}(\Omega)}
$$

pour tout $v \in \mathscr{E}$. Par identification on a $L u=f \in L_{\lambda}^{2}(\Omega)$ et donc $e^{-\lambda t} \gamma u \in$ $H^{-1 / 2}\left(\mathbf{R}^{2}\right)$. Par comparaison avec (B.3.5) on voit que

$$
\forall \varphi \in C_{0}^{\infty}\left(\mathbf{R}^{2}\right) \operatorname{div} \varphi=0, \quad(M \gamma u-h, \varphi)_{L^{3}\left(\mathbf{R}^{2}\right)}=0
$$

et par suite il existe $\phi \in e^{\lambda t} H^{1 / 2}\left(\mathbf{R}^{2}\right)$ tel que $\nabla \phi-M \gamma u=h$. 
Lorsque les coefficients de $L$ et $F$ sont $C^{\infty}$, et lorsque $(f, h) \in C_{0}^{\infty}(\bar{\Omega}) \times C_{0}^{\infty}\left(\mathbf{R}^{2}\right)$, les méthodes classiques de régularisation (cf. par exemple Chazarain-Piriou [3, Chapitre VII, Proposition 6.8]) montrent que $(u, \phi) \in C^{\infty}$ et vérifie les inégalités d'énergie (B.2.2).

Par régularisation de $(f, g)$, puis des coefficients des $(L, F)$, on en tire

Proposition B.3.1. Supposons $q \equiv 0$ Alors il existe $\varepsilon_{0}>0, \lambda_{0}(K)$ et $C_{0}(K)$ tels que si $(L, F)$ satisfait à (B.2.1), alors pour tout $\lambda \geqslant \lambda_{0}(K)$, tout $(f, g) \in L_{\lambda}^{2}(\Omega) \times$ $L_{\lambda}^{2}\left(\mathbf{R}^{2}\right)$ il existe un unique $(u, \phi) \in L_{\lambda}^{2}(\Omega) \times H_{\lambda}^{1}(\Omega)$ solution de (B.3.1). En outre $\gamma u \in L_{\lambda}^{2}\left(\mathbf{R}^{2}\right)$ et l'estimation (B.2.2) est satisfaite.

Enfin, si $(f, g) \subset C_{0}^{\infty}(\bar{\Omega}) \times C_{0}^{\infty}\left(R^{2}\right)$, alors $(u, \phi) \subset C^{\infty}(\bar{\Omega}) \times C^{\infty}\left(R^{2}\right)$.

Maintenant, l'estimation (B.2.2) et un simple argument de perturbation, permettent de supprimer la condition $q \equiv 0$.

Pour achever la démonstration du Théorème 6.2.1, il faut vérifier que, si $(f, g)=0$, pour $t \leqslant h$, il en est de même pour $(u, \phi)$, et obtenir les inégalités d'énergie localisées sur $t \leqslant T,(6.2 .7)$. Ces deux points sont des conséquences classiques de (B.2.2) et du résultat d'existence et d'unicité.

\section{BIBLIOGRAPHIE}

1. S. Alinhac, Le problème de goursat hyperbolique en dimension deux, Comm. Partial Differential Equations 1 (1976), 231-282.

2. J. M. Bony, Calcul symbolique et propagation des singularités pour les équations aux dérivées partielles non linéaires, Ann. Sci. École Norm. Sup (4) 14 (1981), 209-246.

3. J. Chazarain and A. Piriou, Introduction à la théorie des équations aux dérivées partielles, GauthierVillars, Paris, 1981.

4. H. O. Kreiss, Initial boundary value problems for hyperbolic systems, Comm. Pure Appl. Math. 23 (1970), 227-298

5. P. D. Lax, Hyperbolic systems of conservation laws. II, Comm. Pure Appl. Math. 10 (1957), 537-467.

6. Shock waves and entropy; contributions to non linear functional analysis, (E. A. Zarantonello, ed.), Academic Press, New York, 1971.

7. A. Majda, The stability of multi dimensional shock fronts, Mem. Amer. Math. Soc. No. 275 (1983).

8. The existence of multi dimensional shock fronts, Amer. Math. Soc. No. 281 (1983).

9. Y. Meyer, Remarques sur un théorème de J. M. Bony, Supplemento al rendiconti der circolo matematico di Palermo atti del seminario di analisi armonica; Pisa April (1980); série II, No. 1, (1981).

10. , Nouvelles conditions pour les solutions d'équations aux dérivées partielles non linéaires. Séminaire Goulaouic-Schwartz, École Polytechnique, année 1981-1982.

Université de RenNes I, UER Mathematiques and Informatique, Campus de BeaUlieU, 35042, RenNes CEDEX, France 